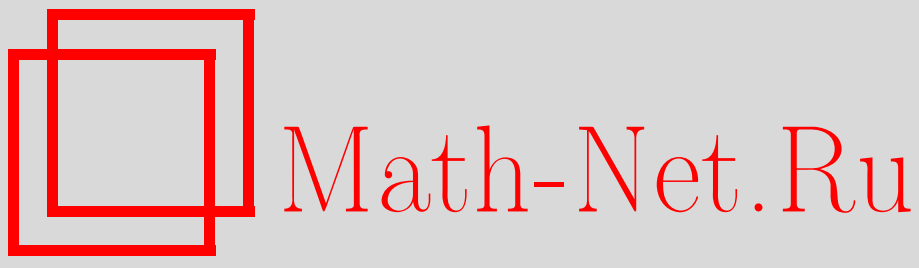

М. М. Сиражудинов, А. Г. Магомедов, В. Г. Магомедова, Краевые задачи для общих эллиптических систем на плоскости. II, Изв. РАН. Сер. матем., 2000, том 64, выпуск 3, 169-224

DOI: https://doi.org/10.4213/im293

Использование Общероссийского математического портала Math-Net.Ru подразумевает, что вы прочитали и согласны с пользовательским соглашением

http://www.mathnet.ru/rus/agreement

Параметры загрузки:

IP: 54.172 .240 .79

26 апреля 2023 г., 14:05:49 
УДК 517.946

М.М. Сиражудинов, А.Г. Магомедов, В. Г. Магомедова

\title{
Краевые задачи для общих эллиптических систем на плоскости. II
}

\begin{abstract}
Для общих эллиптических (по Петровскому, Дуглису и Ниренбергу) систем рассматриваются краевые задачи в ограниченной области плоскости с кусочно гладкой границей. Даются условия, необходимые и достаточные для нётеровости задач, и формулы для индекса. Задачи рассматриваются в весовых пространствах Соболева и Гёльдера.

Библиография: 18 наименований.
\end{abstract}

Началом изучения эллиптических задач в областях с угловыми точками послужила работа [1] для оператора Лапласа. В дальнейшем метод [1] обобщался в различных направлениях [2]-[4]: применительно к краевым задачам теории функций [2], теории упругости [3], теории эллиптических задач [4]. В работах [5]-[7] даются законченные результаты по краевым задачам для обших эллиптических систем на плоскости с постоянными коэффищиентами. В последних даются условия, необходимые и достаточные для нётеровости, и формулы для индекса.

Новый подход к таким задачам (основанный, в частности, на априорных оценках) разработан В. А. Кондратьевым в работе [8]. В дальнейшем на этом пути получены законченные (в смысле условий, необходимых и достаточных для нётеровости) результаты [9], [10]. (Подробную литературу по этим вопросам см. в [10].)

В данной работе рассматриваются краевые задачи для общих эллиптических систем (с переменными коэффициентами) в ограниченной области плоскости с кусочно гладкой границей. Даются достаточно простые (с точки зрения приложений) условия, необходимые и достаточные для нётеровости задачи, и формулы для индекса. Здесь мы обобщаем подход [11], [12] на случай кусочно гладкой области. Отметим, что формулы для индекса общих эллиптических задач до сих пор не известны. Наша работа восполняет этот пробел в двухмерном случае. Мы рассматриваем задачи при условии, что коэффициенты системы принадлежат $C^{\infty}(\bar{Q})$, гладкие части границы $\Gamma_{1}, \ldots, \Gamma_{s}$ из класса $C^{\infty}$ и сужения коэффициентов краевых условий на $\Gamma_{1}, \ldots, \Gamma_{s}$ также из $C^{\infty}$. Эти предположения упрощают формулировки, и, естественно, условия можно значительно ослабить. Например, для справедливости утверждений, касаюшихся задачи Римана-Гильберта из $\S 3-5$, достаточно предположить, что $a, b \in C_{\alpha}^{n}(\bar{Q}), \Gamma_{1}, \ldots, \Gamma_{s} \in C_{\alpha}^{n+1}$ и $\left.G\right|_{\Gamma_{j}} \in C_{\alpha}^{n+1}$.

В $\S 1$ дается описание весовых пространств Соболева и Гёльдера, над которыми рассматриваются задачи. В $\S 2$ получено одно разложение на множители эллиптической матрищы. Основные результаты работы сформулированы в терминах этого разложения. $\S 3$ посвящен условиям, необходимым и достаточным для 
нётеровости задачи Римана-Гильберта для общих эллиптических систем первого порядка. В $\S 4,5$ даются формулы для индекса задачи Римана-Гильберта. В $\S 6$ приведены необходимые и достаточные условия нётеровости краевых задач для общих эллиптических по Дуглису-Ниренбергу систем. $\S 7$ посвящен формулам для любого индекса задачи Пуанкаре для однородных эллиптических систем. В $\S 8$ получены аналогичные формулы для однородных систем, краевые условия которых имеют разные порядки на разных частях границы. Причем наибольший из порядков краевых условий может быть больше порядка системы. В качестве примера дается формула для индекса задачи Дирихле. В последнем $\S 9$ даны формулы для индекса обших эллиптических систем Дуглиса-Ниренберга.

\section{§1. Некоторые пространства функций}

1. Весовые пространства Гёльдера $H_{\mu, \beta}^{n}(Q)[13]$. Пусть $Q$ - область плоскости с кусочно гладкой границей, $\mathscr{J}$ - конечное подмножество граничных точек (куда, в частности, включаются все угловые точки), и пусть

$$
\varrho_{\beta}(x)=\prod_{\tau \in \mathscr{J}}|x-\tau|^{\beta(\tau)}, \quad x \in Q
$$

- весовая функция, где $\beta=\{\beta(\tau) \mid \tau \in \mathscr{J}\}$ - произвольное фиксированное семейство действительных чисел. Семейство $\beta$ называется весовым порядком, линейные операции и неравенства $<, \leqslant$ над весовыми порядками понимаются покомпонентно. В частности, $\varrho_{\beta} \varrho_{\gamma}=\varrho_{\beta+\gamma}$. Когда все $\beta(\tau)$ совпадают, $\beta$ отождествляется с числом, например $\beta=1$ эквивалентно $\beta(\tau)=1, \tau \in \mathscr{J}$.

Обозначим через $H_{\mu, \beta}(Q)$ банахово пространство функций, для которых конечна величина

$$
\left\|u ; H_{\mu, \beta}(Q)\right\|=\left\|\varrho_{-\beta} u\right\|_{0}+\left\{\varrho_{\mu-\beta} u\right\}_{\mu, Q},
$$

где $0<\mu \leqslant 1,\{v\}_{\mu, Q}=\sup _{x, y \in Q}|x-y|^{-\mu}|v(x)-v(y)|,\|v\|_{0}=\sup _{Q}|v(x)|$. Hopма (1.2) в случае ограниченной области $Q$ эквивалентна норме (см. [13, с. 22-23])

$$
\|u\|_{1}=\left\|\varrho_{-\beta} u\right\|_{0}+\sup \varrho_{\mu-\beta}(x)|x-y|^{-\mu}|u(x)-u(y)|,
$$

где $\sup$ берется по всем $x, y \in Q$, для которых $\delta<\varrho_{1}(y) \varrho_{-1}(x)<\delta^{-1}(0<\delta<1-$ любое фиксированное число).

При $\beta=\mu$ пространство $H_{\mu, \mu}(Q)$ совпадает с подпространством $H_{\mu}(Q)$ : $H_{\mu, \mu}(Q)=\left\{u \in H_{\mu}(Q) \mid u_{\mid \mathscr{J}}=0\right\}$. При этом $H_{\mu, \mu}(Q)$-норма эквивалентна $H_{\mu}(Q)$-норме, где $H_{\mu}(Q)$ - обычное пространство Гёльдера.

Определим пространство дифференцируемых функций

$$
H_{\mu, \beta}^{n}(Q)=\left\{u \mid u \in H_{\mu, \beta}^{n-1}(Q), \mathscr{D}_{j} u \in H_{\mu, \beta-1}^{n-1}(Q), \quad j=1,2\right\}
$$


$n=1,2, \ldots$, причем $H_{\mu, \beta}^{0}=H_{\mu, \beta}, \mathscr{D}_{j}=\partial / \partial x_{j}, j=1,2$. Норма в (1.4) определяется равенством

$$
\begin{aligned}
\left\|u ; H_{\mu, \beta}^{n}(Q)\right\| & =\sum_{j=0}^{n} \sum_{i=0}^{j}\left\|\mathscr{D}_{1}^{j-i} \mathscr{D}_{2}^{i} u ; H_{\mu, \beta-j}(Q)\right\| \\
& =\sum_{j=0}^{n} \sum_{i=0}^{j}\left\|\varrho_{j-\beta} \mathscr{D}_{1}^{j-i} \mathscr{D}_{2}^{i} u\right\|_{0}+\sum_{j=0}^{n} \sum_{i=0}^{j}\left\{\varrho_{m+j-\beta} \mathscr{D}_{1}^{j-i} \mathscr{D}_{2}^{i} u\right\}_{\mu, Q} .
\end{aligned}
$$

Относительно этой нормы пространство $H_{\mu, \beta}^{n}(Q)$ банахово.

Пусть $Q$ ограничена; тогда норма (1.5) эквивалентна каждой из следуюших норм

$$
\begin{aligned}
\|u\|_{1} & =\sum_{j=0}^{n} \sum_{i=0}^{j}\left\|\varrho_{j-\beta} \mathscr{D}_{1}^{j-i} \mathscr{D}_{2}^{i} u\right\|_{0}+\sum_{i+j=n}\left\{\varrho_{\mu+n-\beta} \mathscr{D}_{1}^{i} \mathscr{D}_{2}^{j} u\right\}_{\mu, Q}, \\
\|u\|_{2} & =\sum_{j=0}^{n} \sum_{i=0}^{j}\left(\left\|\varrho_{j-\beta} \mathscr{D}_{1}^{j-i} \mathscr{D}_{2}^{i} u\right\|_{0}+\left\{\varrho_{\mu+n-\beta} \mathscr{D}_{1}^{j-i} \mathscr{D}_{2}^{i} u\right\}_{\mu, Q}\right) .
\end{aligned}
$$

С целью получения в дальнейшем априорных оценок для решений краевых задач введем еше одну норму, эквивалентную (1.5) и (1.6). Пусть $K$ - угол с вершиной в точке 0 и раствора $\vartheta, 0<\vartheta<2 \pi$. Через $H_{\beta}^{n}(K), \beta \in \mathbb{R}$, обозначим банахово пространство функций с конечной нормой

$$
\begin{aligned}
\left\|u ; H_{\beta}^{n}(K)\right\|= & \sum_{j=0}^{n} \sum_{i=0}^{j}\left(\sup _{x \in K}\left(|x|^{i-n-\mu}\left|\mathscr{D}_{1}^{j-i} \mathscr{D}_{2}^{i} v(x)\right|\right)\right. \\
& \left.+\sup _{x, y \in K}\left(|x-y|^{-\mu}\left|\mathscr{D}_{1}^{j-i} \mathscr{D}_{2}^{i} v(x)-\mathscr{D}_{1}^{j-i} \mathscr{D}_{2}^{i} v(y)\right|\right)\right),
\end{aligned}
$$

где $v(x)=|x|^{n-\beta+\mu} u(x)$. Пусть $Q-$ ограниченная область плоскости с кусочно гладкой границей, и пусть у каждой точки $\tau \in \mathscr{J}$ есть окрестность $\mathscr{U}^{\tau}$, диффеоморфная кругу $B(\tau)$ с центром в $\tau$. Причем образом пересечения $Q \cap \mathscr{U}^{\tau}$ при этом диффеоморфизме является $B(\tau) \cap K^{\tau}$, где $K^{\tau}$ - угол с вершиной в точке $\tau$ и раствора $0<\vartheta_{\tau}<2 \pi$. Рассмотрим разбиение единицы $\zeta_{\tau} \in C_{0}^{\infty}\left(\mathbb{R}^{2}\right), \tau \in \mathscr{J}$, $\operatorname{supp} \zeta_{\tau} \subset \mathscr{U}^{\tau}, 0<\zeta_{\tau}<1$, причем $\zeta_{\tau}=1$ вблизи угловой точки $\tau \in \mathscr{J}$, и пусть $\zeta_{0}=1-\sum_{\tau \in \mathscr{J}} \zeta_{\tau}$. Требуемая норма определяется равенством

$$
\left\|u ; H_{\mu, \beta}^{n}(Q)\right\|=\left\|\zeta_{0} u ; H_{\mu}^{n}(Q)\right\|+\sum_{\tau \in \mathscr{J}}\left\|\tilde{\zeta}_{\tau} \tilde{u} ; H_{\mu, \beta_{\tau}}^{n}\left(K^{\tau}\right)\right\|,
$$

где $\tilde{\zeta}_{\tau}, \tilde{u}$ - функции, полученные из $\zeta_{\tau}, u$ заменой переменных посредством диффеоморфизма, $H_{\mu}^{n}(Q)$ - обычное пространство Гёльдера $n$ раз непрерьвно дифференцируемых в $\bar{Q}$ функций, $n$-е производные которых удовлетворяют условию Гёльдера. 
2. Пространства $H_{\mu,(\beta)}^{n}(Q)[13]$. Введем банахово пространство $H_{\mu,(\beta)}(Q)$ функций $u(x)$, которые вне любой окрестности $\mathscr{J}(\mathscr{J}$ - конечное множество точек границы) $H_{\mu}$-непрерывны и вблизи $\tau \in \mathscr{J}$ представляются в виде

$$
\begin{gathered}
u(x)=P_{\tau}(x)+u_{\tau}(x), \\
P_{\tau}(x)=\sum_{i+j=0}^{k(\tau)} a_{\tau, i j} x_{1}^{i} x_{2}^{j}, \quad u_{\tau}(x) \in H_{\mu, \beta_{\tau}}\left(Q_{\tau}\right),
\end{gathered}
$$

где $P_{\tau}$ - многочлен переменных $x_{1}, x_{2}$ степени $k(\tau)<\beta_{\tau}$ (в частности, $P_{\tau}=0$ при $\left.\beta_{\tau} \leqslant 0\right), Q_{\tau}-$ криволинейный сектор с вершиной в точке $\tau$. Норму в $H_{\mu,(\beta)}(Q)$ определим равенством

$$
\left\|u ; H_{\mu,(\beta)}(Q)\right\|=\sum_{\tau \in \mathscr{J}}\left(\left\|P_{\tau}\right\|+\left\|\zeta_{\tau} u_{\tau} ; H_{\mu, \beta_{\tau}}\left(Q_{\tau}\right)\right\|\right)+\left\|\zeta_{0} u ; H_{\mu}(Q)\right\|,
$$

где $\zeta_{0}, \zeta_{\tau}, \tau \in \mathscr{J},-$ такие же, как в формуле (1.8), $\left\|P_{\tau}\right\|=\sum_{i+j=0}^{k(\tau)}\left|a_{\tau, i j}\right|$.

Пространство $H_{\mu,(\beta)}$ является конечномерным расширением пространства $H_{\mu, \beta}$ (нульмерным при $\beta \leqslant 0$ ).

Пространства $H_{\mu,(\beta)}^{n}(Q)$ определим аналогично (1.4):

$$
H_{\mu,(\beta)}^{n}(Q)=\left\{u \in H_{\mu,(\beta)}^{n-1}(Q), \quad \mathscr{D}_{1} u, \mathscr{D}_{2} u \in H_{\mu,(\beta-1)}^{n-1}(Q)\right\},
$$

$n=1,2, \ldots$, причем $H_{\mu,(\beta)}^{0}=H_{\mu,(\beta)}$. Норма пространства (1.11) определяется аналогично норме пространства (1.6), исходя из нормы (1.10).

Свойства пространств $H_{\mu, \beta}^{n}(Q), H_{\mu,(\beta)}^{n}(Q)$ объединены в следующих утверждениях.

ПРЕДЛОЖЕНИЕ 1.1 [13], [7]. Пусть $Q$ - ограниченная область. Тогда имеют место утверждения:

а) семейства $\left\{H_{\mu, \beta}^{n}(Q)\right\},\left\{H_{\mu,(\beta)}^{n}(Q)\right\}$ монотонно убывают по всем трем параметрам (т.е. $H_{\mu_{1}, \beta}^{n} \supset H_{\mu_{2}, \beta}^{n}$ nри $\mu_{1} \leqslant \mu_{2}$ u т.д.), оператор умножения $u \mapsto \varrho_{\beta}$ и осуществляет изоморфизм пространств $H_{\mu, \lambda}^{n}(Q) \rightarrow H_{\mu, \lambda+\beta}^{n}(Q)$;

b) вложения $H_{\mu_{2}, \beta}^{n}(Q) \subset H_{\mu_{1}, \beta}^{n}(Q)$ при $\mu_{1}<\mu_{2}, H_{\mu, \beta}^{n}(Q) \subset H_{\mu, \beta}^{n-1}(Q)$ nри $n \geqslant 1$ компактны (то же имеет место и для пространств $\left.H_{\mu,(\beta)}^{n}\right)$;

c) пусть $\mathscr{J}=\{\tau\}$ состоит из одной точки $\tau$ и $Q$ содержстся внутри кругового сектора с иентром в $\tau$ и раствора меньше $2 \pi$, и пусть $w=\ln (z-\tau)$ непрерывная в әтом секторе ветвь логарифма, $\widetilde{Q}-$ образ $Q$ на w-плоскости (в частности, $\widetilde{Q}$ есть полуполоса, когда $Q$ совпадает с сектором круга); тогда отображсение $\tilde{u} \mapsto u(z)=\tilde{u}(\ln (z-\tau))$ осуществляет изоморфизм $H_{\mu}^{n}(\widetilde{Q}) н а H_{\mu, 0}^{n}(Q)$;

d) пусть отображение $\varphi: Q \rightarrow Q_{1}$ такое, что выполнены оценки

$$
|\varphi(x)-\varphi(y)| \leqslant c_{1}|x-y|, \quad|x-\tau| \leqslant c_{2}|\varphi(x)-\varphi(\tau)|,
$$


где $c_{i}>0, \quad i=1,2,-$ константы, $x, y \in Q u \tau \in \mathscr{J}$ любье, и пусть первые производнье функиии $\varphi$ принадлежсат $H_{\mu, 0}^{n-1}$; тогда оператор суперпозиции Tu $=u \circ \varphi$ ограничен из $H_{\mu, \lambda}^{n}\left(Q_{1} ; \mathscr{J}_{1}\right)$ в $H_{\mu, \beta}^{n}(Q ; \mathscr{J})$, где $\mathscr{J}_{1}=\varphi(\mathscr{J})$ и точке $\tau_{1}=\varphi(\tau)$ приписывается вес $\lambda\left(\tau_{1}\right)=\beta(\tau), \quad \tau \in \mathscr{J}$ (в случае пространств $H_{\mu,(\beta)}^{n}$ также имеет место аналогичное утверждение - см. [13]);

е) произведение функиий (как билинейное отображение) ограничено $H_{\mu, \lambda}^{n}(Q) \times H_{\mu, \beta}^{n}(Q) \rightarrow H_{\mu, \lambda+\beta}^{n}(Q)$, при $\lambda \leqslant \beta$ произведение функций ограничено $H_{\mu,(\lambda)}^{n}(Q) \times H_{\mu,(\beta)}^{n}(Q) \rightarrow H_{\mu,(\lambda)}^{n}(Q)$.

Отметим также, что пространство $H_{\mu,(\beta)}^{n}(Q)$ при $\beta=n+\mu$ совпадает (с эквивалентностью норм) с $H_{\mu}^{n}(Q)$.

3. Весовые пространства Соболева $W_{p, \beta}^{n}(Q), W_{p,(\beta)}^{n}(Q)$. Обозначим через $\mathscr{L}_{p, \beta}(Q), 1 \leqslant p<\infty$, пространство Лебега (классов) функций, для которых конечна норма

$$
\|u\|=\left(\int_{Q} \varrho_{-p \beta-2}(x)|u(x)|^{p} d x\right)^{1 / p} .
$$

Относительно $(1.12) \mathscr{L}_{p, \beta}(Q)$ - банахово пространство.

Положим $W_{p, \beta}^{0}(Q)=\mathscr{L}_{p, \beta}(Q)$ и определим пространство $W_{p, \beta}^{n}(Q)$ индукцией:

$$
W_{p, \beta}^{n}(Q)=\left\{u \mid u \in W_{p, \beta}^{n-1}(Q), \quad \mathscr{D}_{i} u \in W_{p, \beta-1}^{n}(Q), i=1,2\right\},
$$

где $n=1,2, \ldots$ При этом норма в (1.13) определяется одной из следуюших формул:

$$
\begin{gathered}
\left\|u ; W_{p, \beta}^{n}(Q)\right\|=\sum_{j=0}^{n} \sum_{i=0}^{j}\left\|\mathscr{D}_{1}^{j-i} \mathscr{D}_{2}^{i} u ; \mathscr{L}_{p, \beta-j}(Q)\right\|, \\
\|u\|_{1}=\left(\sum_{j=0}^{n} \sum_{i=0}^{j}\left\|\mathscr{D}_{1}^{j-i} \mathscr{D}_{2}^{i} u ; \mathscr{L}_{p, \beta-j}(Q)\right\|^{p}\right)^{1 / p}, \\
\|u\|_{2}=\left(\left\|\zeta_{0} u ; W_{p}^{n}(Q)\right\|^{p}+\sum_{\tau \in \mathscr{J}}\left\|\tilde{\zeta}_{\tau} \tilde{u} ; W_{p, \beta_{\tau}}^{n}\left(K^{\tau}\right)\right\|^{p}\right)^{1 / p},
\end{gathered}
$$

где $\zeta_{0}, \zeta_{\tau}, \tau \in \mathscr{J}, K^{\tau}, \tilde{\zeta}_{\tau}, \tilde{u}$ имеют тот же смысл, что и в (1.8). При этом норма в угле определяется по формуле (1.15), где $Q=K^{\tau}, \varrho=|x-\tau|$. Все эти нормы для ограниченной области $Q$ эквивалентны.

Пространство $W_{p,(\beta)}^{n}(Q)$ определим аналогично $H_{\mu,(\beta)}^{n}$. Пусть $W_{p,(\beta)}^{0}(Q)=$ $\mathscr{L}_{p,(\beta)}(Q)$ - класс функций, определенных на $Q$, которые принадлежат пространству $\mathscr{L}_{p}$ вне любой окрестности $\mathscr{J}$, и таких, что вблизи точки $\tau \in \mathscr{J}$ они представляются в виде $(1.9)$, где $u_{\tau} \in \mathscr{L}_{p, \beta_{\tau}}\left(Q_{\tau}\right)$. Относительно нормы

$$
\left\|u ; \mathscr{L}_{p,(\beta)}(Q)\right\|=\sum_{\tau \in \mathscr{J}}\left(\left\|P_{\tau}\right\|+\left\|\zeta_{\tau} u_{\tau} ; \mathscr{L}_{p, \beta_{\tau}}\left(Q_{\tau}\right)\right\|\right)+\left\|\zeta_{0} u ; \mathscr{L}_{p}(Q)\right\|
$$


$\mathscr{L}_{p,(\beta)}(Q)$ - пространство Банаха. В отличие от $(1.9)$ здесь $k(\tau) \leqslant \beta_{\tau}$, где $k(\tau)-$ степень многочлена $P_{\tau}$. Пространство $W_{p,(\beta)}^{n}(Q)$ определим равенством:

$$
W_{p,(\beta)}^{n}(Q)=\left\{u \mid u \in W_{p,(\beta)}^{n-1}, \quad \mathscr{D}_{i} u \in W_{p,(\beta-1)}^{n}, i=1,2\right\},
$$

где $n=1,2, \ldots$ При этом норма в (1.18) определяется формулой

$$
\left\|u ; W_{p,(\beta)}^{n}(Q)\right\|=\left\|\zeta_{0} u ; W_{p}^{n}(Q)\right\|+\sum_{\tau \in \mathscr{J}}\left(\left\|\zeta_{\tau} u ; W_{p, \beta_{\tau}}^{n}\left(K^{\tau}\right)\right\|+\sum_{i+j=0}^{k(\tau)}\left\|\mathscr{D}_{1}^{i} \mathscr{D}_{2}^{j} P_{\tau}\right\|\right),
$$

где под нормой многочлена понимается то же, что в (1.10).

Пространство $W_{p,(\beta)}^{n}(Q)$ совпадает с $W_{p, \beta}^{n}(Q)$ при $\beta<0$ и есть его конечномерное расширение в противном случае.

Свойства введенных пространств объединим в следующее предложение.

ПРЕДЛОЖЕНИЕ 1.2. Пусть $Q$ - ограниченная область. Тогда имеют место утверждения:

а) семейства $W_{p, \beta}^{n}(Q), W_{p,(\beta)}^{n}(Q)$ монотонно убьвают по всем трем параметрам (т.е. $W_{p_{1}, \beta}^{n} \supset W_{p_{2}, \beta}^{n}$ при $p_{1} \leqslant p_{2}$ и т.д.), оператор умножения $u \mapsto \varrho_{\beta}$ и осуществляет изоморфизм пространств $W_{p, \lambda}^{n}(Q) \rightarrow W_{p, \lambda+\beta}^{n}(Q)$;

b) вложсения $W_{p, \beta}^{n}(Q) \subset W_{p, \beta}^{n-1}(Q), \quad n \geqslant 1$, компактны. Пусть $p>2$; тогда при $n \geqslant 1$ имеет место компактное вложение $W_{p, \beta}^{n}(Q) \subset H_{\mu, \lambda}^{n-1}(Q)$, где $\lambda=\beta+\mu-\mu_{0}, \quad 0<\mu<\mu_{0}, \quad \mu_{0}=(p-2) / p$. При $\mu=(p-2) / p$ вложение ограниченное (такие же вложения имеют место и для пространств $W_{p,(\beta)}^{n}$, причем вложение $W_{p,(\beta)}^{n}(Q) \subset H_{\mu,(\lambda)}^{n-1}(Q)$ имеет место для любого $\beta$ такого, что $\left.\beta_{\tau} \notin \mathbb{N} \cup\{0\}\right)$;

c) утверждение с) предложения 1.1 справедливо и в данном случае, если вместо $H_{\mu}^{n}(\widetilde{Q})$ взять $W_{p}^{n}(\widetilde{Q})$ и вместо $H_{\mu, 0}^{n}(Q)$ взять $W_{p,-1 / p}^{n}(Q)$;

d) пусть отображение $\varphi: Q \rightarrow Q_{1}$ такое же, как в d) предложения 1.1; тогда имеет место утверждение, аналогичное d) предложения 1.1;

е) произведение функций (как билинейное отображение) ограничено: $W_{p, \lambda}^{n}(Q) \times W_{p, \beta}^{n}(Q) \rightarrow W_{p, \lambda+\beta}^{n}(Q)$, при $\lambda \leqslant \beta$ произведение функиий ограничено: $W_{p,(\lambda)}^{n}(Q) \times W_{p,(\beta)}^{n}(Q) \rightarrow W_{p,(\lambda)}^{n}(Q)$.

ДокАЗАТЕЛЬСтво. а) Очевидно, что $W_{p, \beta}^{n}, W_{p,(\beta)}^{n}$ монотонно убывают по каждому параметру. Докажем а) в части, касающейся изоморфности. При $n=0$ это есть следствие соотношения $u \varrho_{-\beta-2 / p} \in \mathscr{L}_{p}(Q)$, если $u \in \mathscr{L}_{p, \beta+2 / p}(Q)$. Покажем, как отсюда следует изоморфность при $n=1$. (Этого достаточно для доказательства, ввиду индуктивного способа определения пространств (1.19), (1.24).) Из включения $u \in W_{p, \beta}^{1}(Q)$ следует, что $\varrho_{\lambda} u \in \mathscr{L}_{p, \beta+\lambda}(Q)$. Далее имеем

$$
\begin{aligned}
\varrho_{1-\beta-2 / p} \mathscr{D}_{i} u= & \varrho_{1-(\beta-\lambda)-2 / p} \mathscr{D}_{i}\left(\varrho_{\lambda} u\right) \\
& -\left\{\varrho_{1} \sum_{\tau \in \mathscr{J}} \lambda(\tau) \frac{x_{i}-\tau_{i}}{|x-\tau|^{2}}\right\} \varrho_{-(\beta+\lambda)-2 / p}\left(\varrho_{\lambda} u\right), \quad i=1,2 .
\end{aligned}
$$


Выражение в скобках во втором слагаемом ограничено, тогда ввиду $\varrho_{\lambda} u \in \mathscr{L}_{p, \beta+\lambda}$ получим $\mathscr{D}_{i}\left(\varrho_{\lambda} u\right) \in \mathscr{L}_{p, \beta+\lambda-1}(Q)$. Следовательно, $\varrho_{\lambda} u \in W_{p, \beta+\lambda}^{1}(Q)$. Обратно, если $u \in W_{p, \beta+\lambda}^{1}(Q)$, то аналогично имеем $v=\varrho_{-\lambda} u \in W_{p, \beta}^{1}(Q)$. Значит, $u$ есть образ $v$. Ввиду тривиальности ядра оператора умножения отсюда следует требуемое. (Доказательство для пространства $W_{p,(\beta)}^{n}(Q)$ аналогичное.)

b) Доказательство компактности вложений $W_{p, \beta}^{n} \subset W_{p, \beta}^{n-1}, W_{p,(\beta)}^{n} \subset W_{p,(\beta)}^{n-1}$ аналогично случаю $p=2$ (см. [10, c. 106]), поэтому мы его опускаем. Докажем вторую часть b). Пусть $p>2$. Ввиду индуктивного способа определения пространств достаточно ограничиться случаем $n=1$. Кроме того, ввиду изоморфности оператора умножения из а) ограничимся значением $\beta=(p-2) / p=\mu_{0}$. Так как

$$
\|u\|_{W_{p, \beta}^{1}(Q)}=\left\|\varrho_{-1} u\right\|_{\mathscr{L}_{p}(Q)}+\left\|\mathscr{D}_{1} u\right\|_{\mathscr{L}_{p}(Q)}+\left\|\mathscr{D}_{2} u\right\|_{\mathscr{L}_{p}(Q)}
$$

получим, что $u, \mathscr{D}_{i} u \in \mathscr{L}_{p}(Q)$. Значит, $u \in W_{p}^{1}(Q)$. Тогда по известной теореме вложения для пространств Соболева имеем $u \in C_{\mu}(\widetilde{Q})$, где $0<\mu \leqslant \mu_{0}$. Заметим, что $u$ в угловых точках $\mathscr{J}$ равен нулю. (Иначе первое слагаемое справа в (1.20) ввиду непрерывности $u$ и $p>2$ расходится.) Отсюда следует, что $\sup \left|\varrho_{-\mu} u\right|-$ конечная величина. Следовательно, $u \in H_{\mu, \mu}(Q)$. Оставшаяся часть b) доказывается аналогично.

Доказательства остальных утверждений мы опускаем.

4. Пространства функций на границе области. Нам понадобятся также пространства

$$
H_{\mu, \beta}^{n}(\partial Q \backslash \mathscr{J}), \quad H_{\mu,(\beta)}^{n}(\partial Q \backslash \mathscr{J}), \quad W_{p, \beta}^{n-1 / p}(\partial Q \backslash \mathscr{J}), \quad W_{p,(\beta)}^{n-1 / p}(\partial Q \backslash \mathscr{J})
$$

функций, определенных на гранище области $\bar{Q} \backslash \mathscr{J}$. Они определяются аналогично пространствам функций в области. Пусть $\Gamma$ - гладкая дуга, и пусть $x=\gamma(s)$, $0 \leqslant s \leqslant l$,- естественная параметризация $\Gamma$, где $l$ - длина дуги. Пусть на $\Gamma$ задана функция $u(x)$ точки $x \in \Gamma$. Полагая $x=\gamma(s)$, мы можем рассматривать $u$ как функцию, определенную на отрезке $[0, l]$. Будем говорить, что $u$ принадлежит $H_{\mu, \beta}^{n}(\Gamma)\left(H_{\mu,(\beta)}^{n}(\Gamma)\right)$, если $v(s)=u(x(s))$ принадлежит $H_{\mu, \beta}^{n}(0, l)\left(H_{\mu,(\beta)}^{n}(0, l)\right)$. При этом пространства $H_{\mu, \beta}^{n}(0, l), H_{\mu,(\beta)}^{n}(0, l)$ определяются аналогично соответствуюшему пространству по области (с заменой $Q$ на $(0, l)$ и $\mathscr{J}$ на $\{0 ; l\})$.

Пусть $\Gamma_{1}, \ldots, \Gamma_{k}$, где $k$ - число точек $\mathscr{J},-$ гладкие дуги, составляющие границу $\partial Q, l_{i}$ - длина $\Gamma_{i}$. Говорят, что $u \in H_{\mu, \beta}^{n}(\partial Q \backslash \mathscr{J})$, если сужение $u$ на $\Gamma_{i}, \quad i=1, \ldots, k$, принадлежит $H_{\mu, \beta}^{n}\left(0, l_{i}\right)$. Нормой в $H_{\mu, \beta}^{n}(\partial Q \backslash \mathscr{J})$ будет сумма $H_{\mu, \beta}^{n}\left(0, l_{i}\right)$-норм сужений. Аналогично определяется и пространство $H_{\mu,(\beta)}^{n}(\partial Q \backslash \mathscr{J})$.

Можно дать и непосредственное определение весовых пространств на границе (эквивалентное предыдущему) как пространств сужений (в случае пространств Соболева - следов) элементов из $X(Q)$, где $X(Q)$ - любое из четырех определенных выше весовых пространств по области, на $\partial Q$. В качестве нормы элемента $u \in X(\partial Q \backslash \mathscr{J})$ можно взять $\inf \{\|v ; X(Q)\| \mid v=u$ на $\partial Q \backslash \mathscr{J}\}$. 


\section{§2. Об одном разложении эллиптических матриц}

1. О сглаживании функций и областей. а) Пусть $M$ - ограниченная область плоскости, и пусть $f$ - непрерывная в $\bar{M}$ функция такая, что $f \in H_{\mu}^{n}(\bar{M} \backslash J)$, где $J$ - гладкая незамкнутая кривая с концами на $\partial M, 0<\mu<1, n=0, \ldots,+\infty$ (при $n=+\infty$ можно положить $\mu=0$ ). Тогда для любого $\varepsilon>0$ найдется функция $g_{\varepsilon} \in H_{\mu}^{n}(\bar{M})$ такая, что $g_{\varepsilon}(x)=f(x)$ вне замыкания некоторой окрестности $\mathscr{O}(J)$ кривой $J$ (к окрестности $\mathscr{O}(J)$ мы относим и дуги из $\partial M$, примыкающие к конщам $J)$ и $\left|f(x)-g_{\varepsilon}(x)\right|<\varepsilon, x \in \overline{\mathscr{O}(J)}$. Причем $\mathscr{O}(J)$ не зависит от $\varepsilon$.

Действительно, пусть $\mathscr{O}_{1}$ и $\mathscr{O}_{2}$ - открытые области, покрьваюшие $\bar{M}$, причем $\overline{\mathscr{O}}_{2}$ не имеет общих точек с $J$. И пусть $\vartheta_{1}(x)+\vartheta_{2}(x)=1, x \in \bar{M}$,- разбиение единицы, подчиненное этому покрытию (т.е. $\vartheta_{i} \in C_{0}^{\infty}\left(\mathbb{R}^{2}\right), \operatorname{supp} \vartheta_{i} \in \mathscr{O}_{i}, 0 \leqslant \vartheta_{i}(x) \leqslant 1$, $x \in \mathscr{O}_{i}$ ). Обозначим $\varphi(x)$ гладкую (класса $C^{\infty}$ ) функцию, которая в пересечении $\overline{\mathscr{O}}_{1} \cap \bar{M}$ удовлетворяет оценке $|f(x)-\varphi(x)|<\varepsilon$. Тогда функция $g_{\varepsilon}(x)=\varphi(x) \vartheta_{1}(x)+$ $f(x) \vartheta_{2}(x), \quad x \in \bar{M}$, удовлетворяет всем требованиям, если положим $\mathscr{O}(J)=$ $\mathscr{O}_{1} \cap \bar{M}$.

b) Пусть $A(x)$ - квадратная матрица, принадлежащая $H_{\mu}^{n}(\bar{M} \backslash J) \cap C(\bar{M})$, и пусть $A(x)$ обратима всюду в $\bar{M}$. Тогда ее можно сгладить так, что сглаженная матрица $A_{\varepsilon}(x)$ обратима всюду в $\bar{M}$ и совпадает с $A(x)$ вне $\overline{\mathscr{O}(J)} ; A_{\varepsilon} \in H_{\mu}^{n}(\bar{M})$. (Здесь $\mu, n, \mathscr{O}(J)$ те же, что в п. а); в частности, окрестность $\mathscr{O}(J)$ не зависит от $\varepsilon$.)

Для доказательства заменим каждый элемент $A(x)$ на сглаженную функцию из а). Полученная при этом матрица $A_{\varepsilon}(x)$ для достаточно малого фиксированного $\varepsilon>0$ удовлетворяет требуемым условиям. Невырожденность $A_{\varepsilon}$ следует из очевидного равенства $\operatorname{det} A_{\varepsilon}(x)=\operatorname{det} A(x)+\beta_{\varepsilon}(x)$, где $\beta_{\varepsilon} \rightarrow 0$ равномерно по $x$ при $\varepsilon \rightarrow 0$.

c) Пусть $M$ - ограниченная область с кусочно гладкой границей, причем гладкие куски принадлежат $H_{\mu}^{n}$, где $\mu$ и $n$ такие же, как в а). И пусть $t_{1}, \ldots, t_{n}-$ точки границы, где нарушается гладкость. Границу такой области можно сгладить, не выходя за пределы $M$, так, чтобы:

1) сглаженная граница принадлежала $H_{\mu}^{n}$;

2 ) вне некоторых окрестностей точек $t_{1}, \ldots, t_{n}$ сглаженная граница совпадала с исходной, причем эти окрестности можно взять сколь угодно малыми.

Такое сглаживание произведем следуюшим образом. Примем точку $t_{i}, i=$ $1, \ldots, n$, за начало местной системы координат и ось ординат направим внутрь области $M$ так, чтобы некоторая часть границы, содержашая $t_{i}$ как внутреннюю точку, была графиком вполне определенной функции $\varphi(x), x \in[-a, a]$. В силу предположений о границе $\varphi \in H_{\mu}^{n}([-a, a] \backslash\{0\}) \cap C([-a, a])$. Рассмотрим функцию $\varphi_{\delta} \in$ $C^{\infty}[-a, a],\left|\varphi_{\delta}(x)-\varphi(x)\right|<\delta, x \in[-a, a]$. И пусть $\psi_{\delta}(x)=\varphi_{\delta}(x)+\delta$. График функции $\psi_{\delta}$ расположен внутри $M$, и $\varphi(x)<\psi_{\delta}(x)<\varphi(x)+2 \delta, x \in[-a, a]$. Теперь покроем $[-a, a]$ интервалами $\left(-a_{1},-\varepsilon_{1}\right),(-\varepsilon, \varepsilon),\left(\varepsilon_{1}, a_{1}\right)$, где $a<a_{1}, \varepsilon_{1}<\varepsilon$. И пусть $\vartheta_{1}, \vartheta_{2}, \vartheta_{3} \in C_{0}^{\infty}\left(\mathbb{R}^{2}\right)$ - разбиение единицы $\left(\sum \vartheta_{i}(x)=1, x \in[-a, a]\right)$, подчиненное этому покрытию. Тогда график функции $g(x)=\varphi(x) \vartheta_{1}(x)+\psi_{\delta}(x) \vartheta_{2}(x)+$ $\varphi(x) \vartheta_{3}(x), x \in[-a, a]$, доставляет нам требуемое сглаживание границы. Выполнения условия 2) можно добиться за счет выбора $\varepsilon, \delta, a$. 
2. Об одной факторизации эллиптической матрицы. Пусть $Q$ - ограниченная область с кусочно гадкой границей, причем гладкие части границы принадлежат $H_{\mu}^{n}, 0<\mu<1, n=0, \ldots,+\infty$ (при $n=+\infty$ полагаем $\mu=0$ ). Кроме того, считаем, что граница области распадается на связные замкнутые компоненты $\Gamma_{0}, \ldots, \Gamma_{m}$, гомеоморфные окружности, причем $\Gamma_{0}$ содержит внутри себя остальные. И пусть $\mathscr{B}=\mathscr{B}(x)$ - эллиптическая в $\bar{Q}$ квадратная матрица порядка $k$, принадлежашая $H_{\mu}^{n}(Q)$. (Мы говорим, что матрича әллиптическая на множес$m в е$, если в каждой точке этого множества мнимые части собственных значений отличны от нуля.)

УТВЕРЖДЕНИЕ 2.1. Имеет место следующее разложение:

$$
\mathscr{B}(x)=T(x) \operatorname{diag}\left(\Lambda_{1}(x), \Lambda_{2}(x)\right) T^{-1}(x),
$$

где $T=\left(T_{1}, T_{2}\right), T^{-1^{t}}=\left(\bar{S}^{t}, S^{t}\right)$ - блочные квадратные матрицы порядка $k$ (верхний индекс $t$ означает переход $к$ транспонированной матриче), составленные из двух $\left(k \times k_{+}\right)$- $u\left(k \times k_{-}\right)$-блоков $T_{1}, T_{2} u \bar{S}^{t}, S^{t}$ соответственно. Здесь $k_{+}-$сумма алгебраических кратностей собственных значений матрицы $\mathscr{B}$ из верхней полуплоскости $\operatorname{Im} \lambda>0$, а $k_{-}-$из нижней $\left(k_{+}+k_{-}=k\right)$. Числа $k_{+}$и $k_{-}$не зависят от точек области $\bar{Q}$. Матрищы $\Lambda_{1} u \Lambda_{2}-$ квадратные матрицы порядков $k_{+} u k_{-}$соответственно; $\operatorname{diag}\left(\Lambda_{1}, \Lambda_{2}\right)-$ блочно диагональная матрица с $\Lambda_{1}, \Lambda_{2}$ по диагонали.

Матриць $\Lambda_{1}, \Lambda_{2}, T$ и $T^{-1}$ принадлежат $H_{\mu}^{n}, 0<\mu<1, n=0, \ldots,+\infty$. Спектр матрицы $\Lambda_{1}$ совпадает с соответствующей частью спектра матрицы $\mathscr{B}$ из верхней полуплоскости $\operatorname{Im} \lambda>0$, а матричь $\Lambda_{2}-$ из нижней.

Если әлементы матрицы $\mathscr{B}=\mathscr{B}(x)$ - действительные функции, то из условия әллиптичности следует, что $k_{+}=k_{-}$и $k$-число четное. При этом в разложении (2.1) можно взять $T_{2}=\bar{T}_{1}, \Lambda_{2}=\bar{\Lambda}_{1}$ (черта над матричей означает переход $к$ матриче с комплексно-сопряженными әлементами).

Далее, если $k_{+}=k, \quad k_{-}=0$ (спектр матричь $\mathscr{B}$ расположен в верхней полуплоскости $\operatorname{Im} \lambda>0$ ), разложение (2.1) переходит в тривиальное: $\mathscr{B}=\mathscr{B}$, m.е. $T=E-$ единичная матрица, $\Lambda_{2}$ отсутствует и $\Lambda_{1}=\mathscr{B}$. То же имеет место и для противоположного случая $k_{+}=0, k_{-}=k$. Здесь отсутствует $\Lambda_{1}$, a $\Lambda_{2}=\mathscr{B}$.

Кроме того, разложение (2.1) с указанными свойствами неединственно. Если $\mathscr{B}=\widetilde{T} \operatorname{diag}\left(\tilde{\Lambda}_{1}, \tilde{\Lambda}_{2}\right) \widetilde{T}^{-1}$ - еще одно разложение, имеем

$$
\Lambda_{1}=\mathfrak{C}_{1} \tilde{\Lambda}_{1} \mathfrak{C}_{1}^{-1}, \quad \Lambda_{2}=\mathfrak{C}_{2} \tilde{\Lambda}_{2} \mathfrak{C}_{2}^{-1}, \quad \widetilde{T}=T \operatorname{diag}\left(\mathfrak{C}_{1}, \mathfrak{C}_{2}\right),
$$

где $\mathfrak{C}_{1}, \mathfrak{C}_{2}$ принадлежат $H_{\mu}^{n}(Q)$ так же, как и $\mathscr{B}$. В случае когда $\mathscr{B}$ имеет действительные әлементы, в (2.2) моэсно взять $\mathfrak{C}_{2}=\overline{\mathfrak{C}}_{1}$ (при условии, что для матрицы $\mathscr{B}$ выбраны разложения вида (2.1), где $\Lambda_{2}=\bar{\Lambda}_{1}, \quad \tilde{\Lambda}_{2}=\overline{\tilde{\Lambda}}_{1}$, $\left.T_{2}=\bar{T}_{1}, \quad \widetilde{T}_{2}=\overline{\widetilde{T}}_{1}\right)$. 
ДокАЗАТЕЛЬСТво. Аналогичное утверждение было доказано нами в [11], [12] в случае непрерывных в $\bar{Q}$ матрищ. Укажем, какие изменения следует внести в это утверждение, чтобы получить (2.1).

Матрица $\mathscr{B}(x)$ принадлежит $H_{\mu}^{n}$ в замкнутой области $\bar{Q}$. Следовательно, ее можно продолжить в более широкую область $\bar{Q}_{1}$ с гладкой гранищей с сохранением свойств. Сохранение эллиптичности следует из непрерьвной зависимости собственных значений от матрищы. (Здесь и в дальнейшем под выражениями вида "гладкая гранища" и т.п. понимаются "граница класса $H_{\mu}^{n}$ ” и т.п.) Поэтому, не нарушая обшности, можно считать $Q$ гладкой. Более того, полагаем, что $Q-$ область, ограниченная окружностями. (Общий случай сводится к последнему при помощи конформного отображения [14].)

Пусть $L_{ \pm}$- гладкие замкнутые контуры из верхней $(\operatorname{Im} \lambda \geqslant 0)$ и нижней $(\operatorname{Im} \lambda \leqslant 0)$ полуплоскостей, внутри которых расположены все собственные значения семейства матриц $\{\mathscr{B}(x), x \in \bar{Q}\}$. Такие контуры существуют (см. [12]). Рассмотрим матрищы $\mathscr{P}_{+}, \mathscr{P}_{-}$, определенные формулой

$$
\mathscr{P}_{ \pm}(x)=-\frac{1}{2 \pi i} \int_{L_{ \pm}}(\mathscr{B}(x)-\lambda \mathbf{1})^{-1} d \lambda,
$$

где обход контура $L_{ \pm}$производится против часовой стрелки. Они принадлежат $H_{\mu}^{n}(\bar{Q})$, и $\mathscr{P}_{+}(x)+\mathscr{P}_{-}(x)=\mathbf{1}, x \in \bar{Q},-$ единичная матрища. В [12] построен непрерывный базис $e_{1}(x), \ldots, e_{k_{+}}(x), \ldots, e_{k}(x), x \in \bar{Q}$, пространства $\mathbb{C}^{k}$, где $e_{1}(x), \ldots, e_{k_{+}}(x)$ - базис пространства столбцов матрицы $\mathscr{P}_{+}$, а остальные - матрицы $\mathscr{P}$. . Более того, как следует из [11], [12], этот базис вне некоторых отрезков (соединяюших $\Gamma_{0}, \ldots, \Gamma_{m}$ друг с другом и разбивающих $Q$ на $m$ односвязных областей $\left.Q_{0}, \ldots, Q_{m}\right)$ принадлежит $H_{\mu}^{n}$. Для построения гладкого базиса методом [11] поступим следуюшим образом. Удвоим количество упомянутых отрезков так, чтобы область $Q_{0}$ пересекалась с каждой из $Q_{1}, \ldots, Q_{m}$ по "прямоугольникам" $\Pi_{j}, S_{j}, j=1, \ldots, m$ (ограниченных отрезками параллельных прямых и дугами окружностей $\Gamma_{0}$ и $\Gamma_{j}$ ). Теперь методом [11] построим гладкий базис в каждой из $Q_{0}, \ldots, Q_{m}$. В пересечении $Q_{0} \cap Q_{j}=\Pi_{j} \cup S_{j}$ эти базисы связаны гладкими невырожденными матрищами перехода $\mathscr{A}_{j}(x), \mathscr{C}_{j}(x), j=1, \ldots, m$. Продолжим эти матрицы с границы $\partial\left(\Pi_{j} \cup S_{j}\right)$ внутрь $R_{j}=Q_{j} \backslash\left(\Pi_{j} \cup S_{j}\right)$ аналогично [11]. Тогда мы будем иметь матрицу перехода, гладкую, возможно, за исключением отрезков, принадлежаших $\partial R_{j}$. Сгладим их в соответствии с с) п. 1 . Это, как и в [12], дает нам продолжение (теперь уже гладкое) базиса с $Q_{0}$ на $Q_{j}, j=1, \ldots, m$. Используя этот базис, аналогично [11] получим разложение (2.1) с требуемыми свойствами, что и требовалось доказать.

Для доказательства (2.2) заметим следуюшее. Пространства $C_{ \pm}(x)$ столбцов матриц $\mathscr{P}_{ \pm}(x)$ являются инвариантными подпространствами матрицы $\mathscr{B}$, рассматриваемой как оператор умножения. Кроме того, пространство $\mathbb{C}^{k}$ разлагается в прямую сумму: $\mathbb{C}^{k}=C_{+}(x) \oplus C_{-}(x)$. Следовательно, $\Lambda_{1}$ и $\tilde{\Lambda}_{1}\left(\Lambda_{2}\right.$ и $\left.\tilde{\Lambda}_{2}\right)$ есть матрищы сужения оператора $\mathscr{B}$ на $C_{+}\left(C_{-}\right)$, откуда следует $(2.2)$. Так как при этом матрицы $\mathfrak{C}_{1}, \mathfrak{C}_{2}$ являются матрицами перехода от одного базиса (класса $H_{\mu}^{n}$ ) к другому, то они принадлежат $H_{\mu}^{n}(Q)$. 


\section{§3. Задача Римана-Гильберта в весовых пространствах Соболева и Гёльдера. О нётеровости задачи}

1. Пусть $Q$ - ограниченная область плоскости с кусочно гладкой границей, причем гладкие части границы принадлежат $C^{\infty}$, связные части границы гомеоморфны окружности. Кроме того, считаем, что у каждой точки $\tau \in \mathscr{J}$ (где $\mathscr{J}$ - конечное множество угловых точек границы) есть окрестность $\mathscr{U}^{\tau}$, диффеоморфная открытому кругу $B(\tau)$ с центром в $\tau$. При этом образом пересечения $\mathscr{U}^{\tau} \cap Q$ является $B(\tau) \cap \bar{K}^{\tau}$, где $K^{\tau}$ - угол с вершиной в точке $\tau$ и раствора $\vartheta_{\tau}, 0<\vartheta_{\tau}<2 \pi$. Пусть $x_{\tau}=x_{\tau}(\xi)$-диффеоморфизм. Без ограничения общности можно считать, что матрица Якоби $x_{\tau}^{\prime}$ диффеоморфизма $x_{\tau}=x_{\tau}(\xi)$ равна единичной матрице в точке $\xi=\tau$ (иначе заменим $x_{\tau}$ на $x_{\tau}^{\prime}(\tau) x_{\tau}$ ). Рассмотрим краевую задачу

$$
\begin{aligned}
\mathscr{L} u & \equiv \mathbf{a} \mathscr{D}_{1} u+\mathbf{b} \mathscr{D}_{2} u+\mathbf{c} u=f \in X_{\beta-1}^{n}(Q), \\
G u & =\varphi \in Y_{\beta}^{n}(\partial Q \backslash \mathscr{J}), \quad u \in X_{\beta}^{n+1}(Q),
\end{aligned}
$$

где $X_{\gamma}^{r}(Q)=W_{p, \gamma}^{r}(Q), Y_{\beta}^{n}(\partial Q \backslash \mathscr{J})=W_{p, \beta}^{n+1-1 / p}(\partial Q \backslash \mathscr{J})$, если задача рассматривается в весовых пространствах Соболева, и $X_{\gamma}^{r}(Q)=H_{\mu, \gamma}^{r}(Q), Y_{\beta}^{n}(\partial Q \backslash \mathscr{J})=$ $H_{\mu, \beta}^{n+1}(\partial Q \backslash \mathscr{J})$ в случае пространств Гёльдера, $n \in \mathbb{N} \cup\{0\}, u, f-2 k$-вектор-функции, $\varphi-k$-вектор-функция.

Относительно коэффициентов считаем выполненными следующие условия:

$1^{\circ}$ ) матрицы $\mathbf{a}, \mathbf{b}, \mathbf{c}$ - вешественные квадратные матрицы порядка $2 k, k \geqslant 1$, причем $\mathbf{a}, \mathbf{b} \in C^{\infty}(\bar{Q}), \mathbf{c} \in C^{\infty}(\bar{Q} \backslash \mathscr{J}) \cap \mathscr{L}_{\infty}(Q)$;

$2^{\circ}$ ) система $(3.1)$ эллиптическая в $\bar{Q}$ :

$$
\operatorname{det}\left(\mathbf{a}(x) \xi_{1}+\mathbf{b}(x) \xi_{2}\right) \neq 0, \quad x \in \bar{Q}, \quad \xi \in \mathbb{R}^{2} \backslash 0 ;
$$

$\left.3^{\circ}\right)$ матрица $G$ - вещественная $(k \times 2 k)$-матрица, принадлежащая $C^{\infty}(\bar{Q} \backslash \mathscr{J})$, причем она допускает левое и правое продолжения класса $C^{\infty}$ в точки $\mathscr{J}$; ввиду (3.3) матрица $\mathscr{B}=\mathbf{a}^{-1} \mathbf{b}$ эллиптическая, поэтому для нее имеет место разложение (2.1), где $T_{2}=\bar{T}_{1}, \Lambda_{2}=\bar{\Lambda}\left(\Lambda=\Lambda_{1}\right)$, в силу действительности элементов $\mathscr{B}$;

$4^{\circ}$ ) считаем, что на $\partial Q \backslash \mathscr{J}$ выполнено условие

$$
\operatorname{det} \aleph(x) \neq 0 \quad \text { всюду на } \quad \partial Q \backslash \mathscr{J},
$$

где $\aleph=G \bar{T}_{1}$;

(Как отмечено в [11], условие (3.4) эквивалентно условию дополнительности (условию Шапиро-Лопатинского) для задачи (3.1), (3.2). Разложение (2.1) для заданной эллиптической матрицы неединственно. Последнее не влияет на условие (3.4). Если мы имеем еще одно разложение вида (2.1) с матрицей $\widetilde{T}$ вместо $T$, то согласно $(2.2) \widetilde{T}=T \operatorname{diag}(\mathfrak{C}, \overline{\mathfrak{C}})$, где $\mathfrak{C}-$ невырожденная всюду в $\bar{Q}$ матрица. Следовательно, для второго разложения левая часть (3.4) представится в виде $\operatorname{det} \widetilde{\aleph}=\operatorname{det}(\aleph \mathscr{A})=\operatorname{det} \aleph \operatorname{det} \overline{\mathfrak{C}}$. 


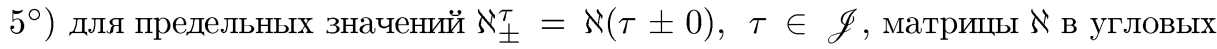
точках справедливы неравенства

$$
\operatorname{det} \aleph_{ \pm}^{\tau} \neq 0, \quad \tau \in \mathscr{J} .
$$

Условие (3.5) эквивалентно условию дополнительности для соответствующей $(3.1),(3.2)$ краевой задаче в угле $K^{\tau}$, а именно для задачи

$$
\begin{gathered}
\mathbf{a}(\tau) \mathscr{D}_{1} u+\mathbf{b}(\tau) \mathscr{D}_{2} u=f \in X_{\beta_{\tau}}^{n}\left(K^{\tau}\right), \\
G_{\tau} u=\varphi \in Y_{\beta_{\tau}}^{n}\left(\partial K^{\tau} \backslash\{\tau\}\right), \quad u \in X_{\beta_{\tau}}^{n+1}\left(K^{\tau}\right),
\end{gathered}
$$

где пространства $X, Y$ имеют тот же смысл, что и в $(3.1),(3.2) ; G_{\tau}=G(\tau+0)$ на "выходящей из $\tau$ " стороне угла, $G_{\tau}=G(\tau-0)$ - на "входящей". ("Выходящая" и "входящая" в смысле положительного обхода границы угла, оставляющем область слева.)

Вопрос нётеровости задачи (3.1), (3.2) тесно связан (см. [10, с. 105-108] - гильбертовый случай) с однозначной разрешимостью задачи в угле (3.6). Для задачи (3.6) известен следующий результат. Рассмотрим краевую задачу

$$
\begin{aligned}
L^{\tau}(\lambda) \equiv & (-\mathbf{a}(\tau) \sin \vartheta+\mathbf{b}(\tau) \cos \vartheta) \frac{d u}{d \vartheta} \\
& -(\mathbf{a}(\tau) \cos \vartheta+\mathbf{b}(\tau) \sin \vartheta)(\lambda-i) u=f \in Z\left(0, \vartheta_{\tau}\right), \\
B^{\tau} u \equiv & \left(\left.G_{+} u\right|_{\vartheta=0},\left.G_{-} u\right|_{\vartheta=\vartheta_{\tau}}\right)=c \in \mathbb{C}^{2 k},
\end{aligned}
$$

где $Z\left(0, \vartheta_{\tau}\right)=W_{p}^{n}\left(0, \vartheta_{\tau}\right) \quad\left(=H_{\mu}^{n}\left(0, \vartheta_{\tau}\right)\right)$. И пусть $\mathfrak{A}^{\tau}(\lambda)=\left(L^{\tau}(\lambda), B^{\tau}\right)$ - соответствуюший (3.7) эллиптический пучок. Обозначим через $\mathscr{F}_{\tau}$ спектр этого пучка. Как известно (см. $[10],[15]), \mathscr{F}_{\tau}$ состоит из не более чем счетного множества изолированных точек $\lambda \in \mathbb{C}$, причем $\mathscr{F}_{\tau}$ не зависит от $n, p, \mu$.

ПРЕДЛОЖЕНИЕ 3.1. Для однозначной разрешимости задачи (3.6) необходимо и достаточно, чтобы на прямой $\operatorname{Re}(i \lambda)=\beta_{\tau}$ не было точек спектра $\mathscr{F}_{\tau}$.

Доказательство этого предложения см. [10, с. 103-104]. При этом нужно учесть, что пространства $V_{p, \gamma}^{n}(K), \Lambda_{\gamma}^{n, \mu}(K)$ из [10] совпадают с пространствами $W_{p, \beta}^{n}(K)$, $H_{\mu, \beta}^{n}(K)$ соответственно при $\gamma=n-\beta-2 / p, \gamma=n-\beta+\mu$.

ЗАМЕЧАНИЕ 3.1. Утверждение предложения 3.1 (в такой же формулировке) имеет место и для более общей задачи в угле $K^{\tau}$, а именно для задачи $(3.1),(3.2)$, где $Q=K^{\tau}$ (см. [10, с. 103-104]).

2. О полунётеровости задачи (3.1), (3.2). Имеет место

Теорема 3.1. Задача Римана-Гильберта (3.1), (3.2) тогда и только тогда полунётеровая с конечномерным ядром, или, что эквивалентно, имеет место априорная оченка

$$
c\left\|u ; X_{\beta}^{n+1}(Q)\right\| \leqslant\left\|\mathscr{L} u ; X_{\beta-1}^{n}(Q)\right\|+\left\|G u ; Y_{\beta}^{n}(\partial Q \backslash \mathscr{J})\right\|+\left\|u ; X_{\beta}^{n}(Q)\right\|
$$

$\forall u \in X_{\beta}^{n+1}(Q)$, где с >0-константа, зависящая только от $\mathbf{a}, \mathbf{b}, \mathbf{c}, G$, когда выполнены условия $\left.\left.1^{\circ}\right)-5^{\circ}\right)$ и условие: на прямой $\operatorname{Re}(i \lambda)=\beta_{\tau}$ нет точек спектра $\mathscr{F}^{\tau}, \tau \in \mathscr{J}$. 
ДоКАЗАТЕЛЬСТВо. В силу компактности вложения $X_{\beta}^{n+1}(Q) \subset X_{\beta}^{n}(Q)$ (см. предложения $1.1,1.2)$ оценка $(3.8)$ влечет полунётеровость с конечномерным ядром оператора задачи (3.1), (3.2) и обратно (см. [16]). Поэтому достаточно установить оценку (3.8). Она получается в точности так же, как аналогичная оценка из $[10$, c. 109] для пространств Соболева с весом при $p=2$. При этом надо воспользоваться $L_{p^{-}}$и $H_{\mu}$-вариантами [17] оценок, используемых в [10]. А для задачи в угле, которая возникает при получении (3.8) методом [10], нужно применить оценку, даваемую предложением 3.1. Необходимость условия доказывается аналогично [10]. Теорема доказана (см. также ниже §6).

\section{3. Некоторые свойства решений. Нётеровость задачи. Имеет место}

Tеорема 3.2. Пусть $\beta_{\tau} \in \mathbb{R}$ такое, что на прямой $\operatorname{Re}(i \lambda)=\beta_{\tau}$ нет точек спектра из $\mathscr{F}_{\tau}, \tau \in \mathscr{J}$. Тогда справедливы утверждения:

а) пусть $и \in X_{\beta}^{n+1}(Q)$ - решение задачи (3.1), (3.2), где $f \in X_{\beta-1}^{n+1}(Q)$, $\varphi \in Y_{\beta}^{n+1}(\partial Q \backslash \mathscr{J}) ;$ тогда $u \in X_{\beta}^{n+2}(Q)$;

b) пусть $u \in W_{p_{1}, \beta}^{n+1}(Q)$ - решение задачи (3.1), (3.2), где $f \in W_{p_{2}, \beta-1}^{n}(Q)$, $\varphi \in W_{p_{2}, \beta}^{n+1-1 / p_{2}}(\partial Q \backslash \mathscr{J})$, причем $1<p_{1} \leqslant p_{2}<\infty ;$ тогда $u \in W_{p_{2}, \beta}^{n+1}(Q)$;

c) пусть $u \in W_{p, \gamma}^{n+1}(Q)$ - решение задачи (3.1), (3.2), где $f \stackrel{p_{2}, \beta}{\in} H_{\mu, \beta-1}^{n}(Q)$, $\varphi \in H_{\mu, \beta}^{n+1}(\partial Q \backslash \mathscr{J})$, причем $\gamma=\beta-2 / p ;$ тогда $u \in H_{\mu, \beta}^{n}(Q)$ для достаточно больиих $p$.

ДоказАтЕЛЬСтво. а) Пусть $\zeta_{0}, \zeta_{\tau}, \tau \in \mathscr{J},-$ разбиение единицы из определений норм (1.8), (1.16). Рассмотрим вектор $v=\zeta_{0} u$, и пусть $Q_{0}$ - пересечение носителя $\zeta_{0}$ с $Q$. Очевидно, в $Q_{0}$ вектор $v$ удовлетворяет системе $\mathscr{L} v=g$, $g=\zeta_{0} f+a u \mathscr{D}_{1} \zeta_{0}+b u \mathscr{D}_{2} \zeta_{0}$. При этом $g \in W_{p}^{n+1}\left(Q_{0}\right)$ (или $\left.g \in H_{\mu}^{n+1}\left(Q_{0}\right)\right)$. На пересечении $\Gamma=\partial Q_{0} \cap \partial Q$ имеем $G v=\zeta_{0} \varphi \in W_{p}^{n+2-1 / p}(\Gamma)\left(\in H_{\mu}^{n+2}(\Gamma)\right)$. Отсюда в силу известных оценок Шаудеровского типа [17] получим $u \in W_{p}^{n+2}\left(Q_{1}\right)$ $\left(\in H_{\mu}^{n+2}\left(Q_{1}\right)\right)$, где $Q_{1}$ - любая замкнутая подобласть $\bar{Q} \backslash \mathscr{J}$. Следовательно, согласно определению норм (1.8), (1.16) для завершения доказательства остается показать, что $u \in W_{p, \beta}^{n+2}\left(\in H_{\mu, \beta}^{n+2}\right)$ в окрестности угловых точек. Последнее будет доказано, если мы получим аналогичное теореме свойство для угла $K^{\tau}, \tau \in \mathscr{J}$.

После замены $x_{1}=x_{1}^{\tau}\left(\xi_{1}, \xi_{2}\right), x_{2}=x_{2}^{\tau}\left(\xi_{1}, \xi_{2}\right)$, где $x=x_{\tau}(\xi)$ - диффеоморфизм (см. начало настояшего параграфа и определения норм $(1.8),(1.16))$, мы получим, что $v=\zeta_{\tau} u$ (продолженная нулем вне носителя $\zeta_{\tau}$ ) является решением задачи в угле

$$
\begin{gathered}
\mathbf{a}_{1} \frac{\partial v}{\partial \xi_{1}}+\mathbf{b}_{1} \frac{\partial v}{\partial \xi_{2}}=g, \\
G_{1} v=\varphi_{1},
\end{gathered}
$$

где $\mathbf{a}_{1}(\xi)=\mathbf{a} \mathscr{D}_{1} \xi_{1}+\mathbf{b} \mathscr{D}_{2} \xi_{1}, \mathbf{b}_{1}(\xi)=\mathbf{a} \mathscr{D}_{1} \xi_{2}+\mathbf{b} \mathscr{D}_{2} \xi_{2}, g=\zeta_{\tau} f+a_{1} u D_{1} \zeta_{\tau}+\mathbf{b}_{1} u D_{2} \zeta_{\tau}$, $\varphi_{1}=\zeta_{\tau} \varphi$. При этом $g \in X_{\beta_{\tau}-1}^{n+1}\left(K^{\tau}\right), \varphi_{1} \in Y_{\beta}^{n+1}\left(\partial K^{\tau} \backslash\{\tau\}\right)$. (Предполагаем, что $g, \varphi_{1}, v$ финитно продолжены на все $K^{\tau}$.) Применим замечание 3.1 (где $n$ заменен на $n+1)$ к задаче (3.9); тогда получим, что она имеет единственное решение $v_{1} \in X_{\beta_{\tau}}^{n+2}\left(K^{\tau}\right)$. В силу вложения $X_{\beta_{\tau}}^{n+2}\left(K^{\tau}\right) \subset X_{\beta_{\tau}}^{n+1}\left(K^{\tau}\right)$ отсюда следует, что $v_{1}$ является единственным решением той же задачи, где $n$ заменено на $n+1$. С другой 
стороны, таким решением является $v=\zeta_{\tau} u$ (продолжено нулем вне носителя $\zeta_{\tau}$ ). Значит, $v_{1}=\zeta_{\tau} u$ и $\zeta_{\tau} u \in X_{\beta_{\tau}}^{n+2}\left(K^{\tau}\right)$.

b) Как и в а), здесь дело сводится к изучению поведения решения вблизи угловой точки $\tau \in \mathscr{J}$. Вектор-функция $v=\zeta_{\tau} u$ (как и в а)) является решением задачи (3.9) в угле $K^{\tau}$. После замены $w=\ln (z-\tau)$, где $\ln (z-\tau)$-непрерывная ветвь логарифма $\left(z=x_{1}+i x_{2}, \quad\left(x_{1}, x_{2}\right)=x\right)$, задача в угле переходит в задачу в полосе П (с равенством норм). Тогда согласно [10, с. 100, следствие 6.5] получим, что $v \in$ $W_{p_{2}, \beta}^{n+1}(\Pi)$ (см. также [10, с.87]). При отображении $w=\ln (z-\tau)$ сектор $K_{\delta}^{\tau}$ (где сосредоточена функция $\zeta_{\tau}$ ) переходит в полуполосу $\Pi_{\delta} \subset \Pi$ и $v=0$ в $\ \backslash \Pi_{\delta}$. Согласно предложению $1.2, \mathrm{a})$, с) отсюда следует, что $v \in W_{p_{2}, \beta}^{n+1}\left(K^{\tau}\right)$.

Доказательство утверждения с) проводится аналогично b). При этом надо воспользоваться свойством решений в полосе из [10, с. 103]. Теорема доказана.

Используем теорему 3.2 для описания ядра оператора краевой задачи (3.1), (3.2) и ядра сопряженного оператора.

ТЕОРема 3.3. В условиях теоремы 3.1 ядро оператора задачи (3.1), (3.2) $u$ ядро сопряженного оператора не зависят от $n, p, \mu \quad(n=0,1, \ldots ; 1<$ $p<+\infty ; 0<\mu<1)$. Кроме того, эти ядра не меняются при переходе от (весовых) пространств Соболева к пространствам Гёльдера.

ДоказАтельство. Пусть $\mathscr{A}$ - оператор задачи $(3.1),(3.2), \mathscr{A}^{*}$ - сопряженный оператор. Независимость ядра $\operatorname{Ker} \mathscr{A}$ от $n$ следует из теоремы $3.2, \mathrm{a})$, от $p-$ из b). Пусть $0<\mu<1$ - любое фиксированное число, и пусть $\gamma=\beta-2 / p$. Если на прямой $\operatorname{Re}(i \lambda)=\beta_{\tau}$ нет точек спектра $\mathscr{F}_{\tau}$, при достаточно больших $p$ этим же свойством обладает и $\gamma_{\tau}=\beta_{\tau}-2 / p$. Фиксируем любое такое $p$. Тогда согласно теореме $3.2, \mathrm{c})$, вложению $H_{\mu, \beta}^{n}(Q) \subset W_{p, \gamma}^{n}(Q)$ и доказанной части теоремы получим независимость ядра $\operatorname{Ker} \mathscr{A}$ от $\mu$.

Пусть $\mathscr{A}_{k}^{*}$ и $\mathscr{A}_{m}^{*}$ - операторы, сопряженные операторам $\mathscr{A}_{k}$ и $\mathscr{A}_{m}$ задачи (3.1), (3.2) при $n=k$ и $n=m$, и пусть $k>m$. Оператор $\mathscr{A}_{k}$ при $k>m$, очевидно, есть сужение $\mathscr{A}_{m}$. Поэтому $\mathscr{A}_{k}^{*}$ есть расширение $\mathscr{A}_{m}^{*}$, значит, Ker $\mathscr{A}_{m}^{*} \subset \operatorname{Ker} \mathscr{A}_{k}^{*}$. Покажем, что здесь точное равенство. Если это не так, найдется вектор $F=$ $\left(f_{0}, \varphi_{0}\right)$, принадлежаший $X_{\beta-1}^{k}(Q) \times Y_{\beta}^{k}(\partial Q \backslash \mathscr{J})$, “ортогональный” $\operatorname{Ker} \mathscr{A}_{m}^{*}$, но не $\operatorname{Ker} \mathscr{A}_{k}^{*}$. Ввиду полунётеровости задачи (3.1), (3.2) (теорема 3.1) она нормально разрешима. Поэтому сушествует решение $u$ задачи $(3.1),(3.2)$, где $f=f_{0}, \varphi=$ $\varphi_{0}, n=m$, принадлежащее $X_{\beta}^{m+1}$. Согласно теореме 3.2, а) отсюда имеем $u \in$ $X_{\beta}^{k+1}(Q)$, т.е. задача $(3.1),(3.2)$ разрешима и при $n=k$. Это противоречит неортогональности $F$ ядру $\operatorname{Ker} \mathscr{A}_{k}^{*}$.

Итак, независимость $\operatorname{Ker} \mathscr{A}^{*}$ от $n$ установлена. Аналогично доказывается независимость и от $p$. Пусть $0<\mu<1$ - любое фиксированное число, и пусть $p_{0}=2 /(1-\mu)$. Рассмотрим оператор $\mathscr{A}_{1}$ задачи $(3.1),(3.2)$ в $L_{p}$-пространствах с $n=1, p=p_{0}$. Согласно предложению $\left.1.2, \mathrm{~b}\right)$ имеет место вложение $W_{p_{0}, \beta}^{k} \subset H_{\mu, \beta}^{k-1}$ для любого $k \in \mathbb{N}$. Пусть $\mathscr{A}_{0}$ - оператор задачи $(3.1),(3.2)$ в $H$-пространствах с $n=0$. С учетом отмеченных вложений, как и выше, получим Ker $\mathscr{A}_{1}^{*}=\operatorname{Ker} \mathscr{A}_{0}^{*}$. Отсюда и из доказанной части теоремы следует независимость Ker $\mathscr{A}^{*}$ от $\mu$. По- 
следняя часть теоремы доказывается с привлечением отмеченных выше операторов $\mathscr{A}_{1}$ и $\mathscr{A}_{0}$. Теорема доказана.

Теорема 3.4. Для нётеровости задачи Римана-Гильберта (3.1), (3.2) необходимо и достаточно выполнения условий $\left.1^{\circ}\right)-5^{\circ}$ ) и условия: на прямой $\operatorname{Re}(i \lambda)=\beta_{\tau}$ нет точек спектра $\mathscr{F}_{\tau}, \tau \in \mathscr{J}$. При выполнении этих условий индекс задачи не зависит от $n, p, \mu \quad(n=0,1, \ldots ; 1<p<+\infty ; 0<\mu<1)$, а также от пространств, над которыми рассматривается задача (они могут быть как весовыми пространствами Соболева, так и Гёльдера).

Пусть выполнены условия $\left.\left.1^{\circ}\right)-5^{\circ}\right)$, и пусть на прямых $\operatorname{Re}(i \lambda)=\beta_{\tau}$, $\operatorname{Re}(i \lambda)=\gamma_{\tau}, \beta_{\tau} \leqslant \gamma_{\tau}$, нет точек спектра $\mathscr{F}_{\tau}, \tau \in \mathscr{J}$. Тогда индексы операторов краевой задачи (3.1), (3.2), отвечающих $\beta$ и $\gamma$, связаны равенством

$$
\text { ind } \mathscr{A}_{\beta}=\text { ind } \mathscr{A}_{\gamma}+\sum_{\tau \in \mathscr{J}} S_{\tau}
$$

где $S_{\tau} \geqslant 0$ - целое, равное сумме полных кратностей всех собственных значений пучка $\mathfrak{A}_{\tau}(\lambda)$, попавших внутрь полосы $\beta_{\tau}<\operatorname{Re}(i \lambda)<\gamma_{\tau}$.

ДокАЗАТЕЛЬСтво следует из теорем 3.1-3.3 с учетом того, что при $p=2$ задача нётеровая (см. [10, с. 108]). Формула (3.10) при $p=2$ также известна [10, с. 122].

\section{§4. Формула для индекса задачи Римана-Гильберта}

1. Формула А. П. Солдатова [7]. Рассмотрим задачу Римана-Гильберта:

$$
\begin{gathered}
\mathscr{L} u \equiv \mathbf{a} \mathscr{D}_{1} u+\mathbf{b} \mathscr{D}_{2} u=f \in H_{\mu,(\beta-1)}(Q), \\
G u=\varphi \in H_{\mu,(\beta)}^{1}(\partial Q \backslash \mathscr{J}), \quad u \in H_{\mu,(\beta)}^{1}(Q),
\end{gathered}
$$

где $\mathbf{a}, \mathbf{b}$ - матрищы порядка $2 N$, элементы которых - действительные числа, причем вьполнено условие эллиптичности (3.3).

Эллиптическая матрица $B=-\mathbf{b}^{-1} \mathbf{a}$ представима в жордановой форме:

$$
B=\mathscr{T} \operatorname{diag}(J, \bar{J}) \mathscr{T}^{-1}
$$

где $\mathscr{T}=\left(\mathscr{T}_{1}, \overline{\mathscr{T}}_{1}\right)$ имеет ту же структуру, что и $T$ из $(2.1)$. (Очевидно, что

$$
\mathbf{a}^{-1} \mathbf{b}=\mathscr{T} \operatorname{diag}(\Lambda, \bar{\Lambda}) \mathscr{T}^{-1}
$$

где $\Lambda=-J^{-1}, \mathscr{T}$ - матрица из (4.3). Так как собственные значения $\Lambda$ расположены в верхней полуплоскости, (4.4) является одним из разложений (2.1) матрицы $\mathbf{a}^{-1} \mathbf{b}$.) Предположим также, что для задачи (4.1), (4.2) выполнены условия $\left.\left.2^{\circ}\right)-5^{\circ}\right)$ из $\S 3$ с $\mathscr{T}$ из $(4.3)$ вместо $T$.

Напомним некоторые понятия и обозначения из работ [6], [7]. 
Пусть $\left(\Gamma_{1}, \ldots, \Gamma_{s}\right)$ - гладкие (класса $\left.C^{\infty}\right)$ дуги, составляющие гранищу $\partial Q$, причем $s=|\mathscr{J}|$ - число угловых точек. Наряду с обычной топологией для удобства введем на $\partial Q$ составную топологию, в которой концы всех дуг $\Gamma_{j}$ считаются различными. Занумеруем последние $\tau_{1}, \ldots, \tau_{2 s}$, считая $\tau_{2 j-1}$ и $\tau_{2 j}$ конщами $\Gamma_{j}$ $\left(\tau_{2 j-1}-\right.$ начало, $\tau_{2 j}$ - конец). В составной топологии каждая дуга есть связная компонента и $\partial Q=\bigoplus_{1}^{s} \Gamma_{j}$. Функция $\aleph(x)$, непрерывная на $\oplus \Gamma_{j}$, кусочно непрерывна в обычной топологии $\partial Q$ с односторонними пределами $\aleph\left(\tau_{i}\right), i=1, \ldots, 2 s$, в точках $\mathscr{J}$.

Множество номеров $1, \ldots, 2 s$ разбиваем на $s=|\mathscr{J}|$ пар $\mathscr{P}_{1}, \ldots, \mathscr{P}_{s}$ таким образом, что для пары $\mathscr{P}_{j}=\{k, r\}$ конщы $\tau_{k}$ и $\tau_{r}$ "лежат над" точкой $\tau_{(j)}=\tau \in \mathscr{J}$. Пусть $Q_{\tau} \subset Q$ - криволинейньй сектор с вершиной в точке $\tau=\tau_{(j)} \in \mathscr{J}$. Его раствор обозначим $\vartheta_{j}$, и пусть $0<\vartheta_{j}<2 \pi$. Единичные векторы касательных к боковым сторонам сектора $Q_{\tau}$ в точке $\tau=\tau_{(j)}$ обозначим $q_{k}$ и $q_{r}$. Точнее, $q_{k}=a\left(\tau_{k}\right)$, $q_{r}=a\left(\tau_{r}\right)$, где $a(z)=(z-\tau) /|z-\tau|, z \in \partial Q_{\tau}$. Пара $\mathscr{P}_{j}=\{k, r\}$ в дальнейшем упорядочена условием

$$
\arg q_{k}-\arg q_{r}=\vartheta_{j}, \quad \mathscr{P}_{j}=\{k, r\}
$$

Пусть $q^{\zeta}(\lambda) \stackrel{\text { def }}{=}\left\{\left(\operatorname{Re} q_{k}+\lambda \operatorname{Im} q_{k}\right) /\left(\operatorname{Re} q_{r}+\lambda \operatorname{Im} q_{r}\right)\right\}^{\zeta}, \lambda \in \mathbb{C},-$ функция от $\lambda$, причем ветвь степенной функции фиксирована условием (4.5). Введем квадратную матрицу порядка $N$ :

$$
v_{j}(\zeta)=\left\{\left(\operatorname{Re} q_{k}+J \operatorname{Im} q_{k}\right)\left(\operatorname{Re} q_{r}+J \operatorname{Im} q_{r}\right)^{-1}\right\}^{\zeta}=q^{\zeta}(J)
$$

Матрица (4.6) по определению есть значение функции от матрищы $f(u)=q^{\zeta}(u)$ при $u=J$. Определим в точке $\tau_{j}=\tau \in \mathscr{J}$ символ $x_{j}(\zeta)$ Солдатова [7] равенством

$$
x_{j}(\zeta)=\mathbf{1}-c^{-1}\left(\tau_{j}-0\right) v_{j}(\zeta) c\left(\tau_{j}+0\right) \bar{v}_{j}^{-1}(\zeta)
$$

где $c=\bar{\aleph}^{-1} \aleph, \aleph=G \overline{\mathscr{T}}_{1}, \bar{v}_{j}(\zeta) \stackrel{\text { def }}{=} \overline{v_{j}(\bar{\zeta})}$. Положим

$$
y_{j}(\zeta)=\left(\mathbf{1}+R_{j}\right)\left(\mathbf{1}-v_{j} \bar{v}_{j}^{-1}\right)
$$

где $\mathbf{1}$ - единичная матрица, $R_{j}$ - любая фиксированная исчезающая на $\infty$ рациональная матрица-функция такая, что:

(i) $y_{j}(\zeta)$ аналитична на всей плоскости и $\operatorname{det} y_{j}(\zeta) \neq 0$ при $|\operatorname{Re} \zeta|<M$ с некоторым достаточно большим $M>0$;

(ii) в полуплоскости $\operatorname{Re} \zeta<0$ функция $\operatorname{det}\left(\mathbf{1}+R_{j}(\zeta)\right)$ имеет одинаковые числа (включая кратности) нулей и полюсов.

Пусть $\beta_{\tau} \notin \mathbb{N} \cup\{0\}, \tau \in \mathscr{J}$; тогда справедлива 
Лемма 4.1 [6], [7]. Задача (4.1), (4.2) тогда и только тогда нётеровая, когда кроме условий $\left.1^{\circ}\right)-5^{\circ}$ ) выполнено условие: $\operatorname{det} x_{j}(\zeta) \neq 0, \operatorname{Re} \zeta=\beta_{\tau}$, $\tau=\tau_{j} \in \mathscr{J}$. При выполнении этих условий индекс задачи дается равенством

$$
\text { ind } \mathscr{A}=\operatorname{Ind}_{\Gamma} \bar{\aleph}^{-1} \aleph-\sum_{j=1}^{|\mathscr{F}|} \operatorname{Ind}_{\beta_{j}} x_{j}(\zeta) y_{j}^{-1}(\zeta)-N(m-1)
$$

¿əe

$$
\begin{gathered}
\operatorname{Ind}_{\Gamma} \bar{\aleph}^{-1} \aleph=\frac{1}{2 \pi i} \sum_{l=1}^{2 s}(-1)^{l} \ln \operatorname{det}\left(\bar{\aleph}^{-1} \aleph\right)\left(\tau_{l}\right), \\
\operatorname{Ind}_{\beta_{j}} x_{j}(\zeta) y_{j}^{-1}(\zeta)=\left.(2 \pi i)^{-1} \ln \left(\operatorname{det}\left(x_{j}(\zeta) y_{j}^{-1}(\zeta)\right)\right)\right|_{\beta_{j}-i \infty} ^{\beta_{j}+i \infty}
\end{gathered}
$$

(ln $\operatorname{det} \bar{\aleph}^{-1} \aleph-$ - непрерывная на $\oplus \Gamma_{j}$ ветвь логарифма, аналогичный смысл имеет и второй логарифм), $m+1$ - число связных компонент границы $\partial Q,|\mathscr{J}|-$ число точек $\mathscr{J}, \beta_{j}=\beta_{\tau_{j}}$.

Пусть $\beta=\beta_{\tau}$ и $\gamma=\gamma_{\tau}$ - допустимые весовые порядки, т.е. на прямых $\operatorname{Re} \zeta=\beta_{\tau}, \operatorname{Re} \zeta=\gamma_{\tau}, \tau \in \mathscr{J}$, нет нулей определителя концевого символа, и пусть выполнены условия $\left.2^{\circ}\right)-5^{\circ}$ ) из §3. Тогда индексы задачи (4.1), (4.2) связаны равенством

$$
\text { ind } \mathscr{A}_{\gamma}-\text { ind } \mathscr{A}_{\beta}=\sum_{\tau \in \mathscr{J}} S_{\tau}
$$

где $\mathscr{A}_{\gamma}$ - оператор задачи (4.1), (4.2) при $\beta=\gamma, \pm S_{\tau}$ равен числу нулей концевого символа $x_{j}(\zeta), \tau_{j}=\tau \in \mathscr{J}$ (с учетом кратности) между прямыми $\operatorname{Re} \zeta=\beta_{\tau}, \operatorname{Re} \zeta=\gamma_{\tau}$. Знак "-" соответствует случаю $\beta_{\tau} \leqslant \gamma_{\tau}$, а знак "+" случало $\beta_{\tau} \geqslant \gamma_{\tau}$.

ДокАЗАТЕЛЬСТво. Формула (4.8) получена Солдатовым [7] для однородной системы (4.1) $(f=0)$ в случае, когда $\varphi \in H_{\mu,(\beta)}(\partial Q \backslash \mathscr{J})$. В условиях (4.2) индекс дается той же формулой. Устанавливается это методом теоремы 3.3 .

Покажем, что в (4.1) без ограничения общности можно считать, что $f=0$. После замены $u=\mathscr{T}\left(\frac{w}{w}\right)$, где $\mathscr{T}$ из (4.3), задача (4.1), (4.2) становится эквивалентной следующей:

$$
\begin{gathered}
\mathscr{D}_{2} w-J \mathscr{D}_{1} w=g \in H_{\mu,(\beta-1)}(Q), \\
\operatorname{Re}\left(G \mathscr{T}_{1} w\right)=\varphi \in H_{\mu,(\beta)}^{1}(\partial Q \backslash \mathscr{J}), \quad w \in H_{\mu,(\beta)}^{1}(Q),
\end{gathered}
$$

где $g=\bar{B} f,\left(\begin{array}{l}\bar{B} \\ B\end{array}\right)=\mathscr{T}^{-1}$. Поэтому достаточно показать, что в $(4.10)$ можно положить $g=0$.

Пусть $\left(L_{1}, \ldots, L_{m}\right)$ - связные компоненты границы $\partial Q$, причем $L_{0}$ содержит внутри себя все остальные. Рассмотрим матрищу

$$
\Phi(z)=\prod_{\tau \in \mathscr{J}} \Upsilon_{\tau}(z)
$$


где $\Upsilon_{\tau}(z)=[z-\tau]^{\gamma(\tau)}$ при $\tau \in L_{0}, \Upsilon_{\tau}(z)=[z-\tau]^{\gamma(\tau)}\left[z-z_{j}\right]^{-\gamma(\tau)}$ при $\tau \in L_{j}$. Здесь $z=x_{1}+i x_{2}, z_{j}$ - точка из дополнения $Q$, расположенная внутри $L_{j},[\zeta]=$ $\zeta_{1} \mathbf{1}+\zeta_{2} J, \zeta=\zeta_{1}+i \zeta_{2}$. Берутся фиксированные ветви степенных функций. Матрица $\Phi(z)-J$-аналитическая матрица-функция, т.е. она является решением системы

$$
\mathscr{L} \Phi \equiv \mathscr{D}_{2} \Phi-J \mathscr{D}_{1} \Phi=0
$$

Кроме того, $\Phi \in H_{\mu, \gamma}^{j}(Q)$ для любого $j \in \mathbb{N} \cup 0, \beta_{1}=\gamma(\tau)$ (см. [7, с. 575-576]). Тогда ввиду предложения $1.1 \Phi w \in H_{\mu,(\mu+1)}^{1}(Q), \Phi g \in H_{\mu,(\mu)}(Q)$ при $\gamma=\mu+$ $1-\beta$. Поэтому, умножив (4.10) слева на матрищу $\Phi(z)$, с учетом (4.11) получим, что в (4.10) можно считать $w \in H_{\mu,(\mu+1)}^{1}(Q), g \in H_{\mu,(\mu)}(Q)$. Кроме того, здесь можно опустить $(\mu+1),(\mu)$, так как $H_{\mu,(\mu+1)}^{1}=H_{\mu}^{1}, H_{\mu,(\mu)}=H_{\mu}$, что и предполагаем ниже. Продолжим элементы $H_{\mu}(Q)$ финитно на всю плоскость так, чтобы продолжения принадлежали $H_{\mu}\left(\mathbb{R}^{2}\right)$ и равнялись нулю вне некоторой области $Q_{1}$, $\bar{Q} \subset Q_{1}$. Матрица $E=\left(x_{1} \mathbf{1}+x_{2} J\right)^{-1}$ является фундаментальным решением системы (4.11), т.е. $\mathscr{L} E=\delta(x) \mathbf{1}$. Поэтому свертка $u=E * g$ является частным решением системы (4.10), принадлежащим $H_{\mu}^{1}(Q)$. Теперь после замены $w \mapsto \omega+w$ задача (4.10) становится эквивалентной задаче с $g=0$. Лемма доказана.

2. Для получения формулы для индекса задачи Римана-Гильберта (3.1), (3.2) в терминах конщевого символа Солдатова $x_{j}(\zeta)$ из п. 1 нам нужно уточнить понятие концевого символа применительно к системе с переменными коэффициентами. Здесь мы этот символ обозначим $\mathscr{X}_{j}(\zeta)$ и определим по формуле

$$
\begin{gathered}
\mathscr{X}_{j}(\zeta)=\mathbf{1}-C^{-1}\left(\tau_{j}-0\right) V_{j}(\zeta) C\left(\tau_{j}+0\right) \bar{V}_{j}^{-1}(\zeta), \\
V_{j}(\zeta)=\left\{\left(\operatorname{Im} q_{k}-\Lambda \operatorname{Re} q_{k}\right)\left(\operatorname{Im} q_{r}-\Lambda \operatorname{Re} q_{r}\right)^{-1}\right\}^{\zeta \text { def }}=\mathfrak{q}^{\zeta}(\Lambda),
\end{gathered}
$$

где $C=\bar{\aleph}^{-1} \aleph, \aleph=G \bar{T}_{1}, \bar{V}_{j}(\zeta) \stackrel{\text { def }}{=} \overline{V_{j}(\bar{\zeta})}$. Здесь $T=\left(T_{1}, \bar{T}_{1}\right), \Lambda$ - матрицы из разложения (2.1) эллиптической матрищы $\mathbf{a}^{-1} \mathbf{b}$, вычисленные в точке $\tau=\tau_{j} \in \mathscr{J}$.

Рассмотрим задачу Римана-Гильберта:

$$
\begin{gathered}
\mathscr{L} u \equiv \mathbf{a} \mathscr{D}_{1} u+\mathbf{b} \mathscr{D}_{2} u+\mathbf{c} u=f \in X_{(\beta-1)}^{n}(Q), \\
G u=\varphi \in Y_{(\beta)}^{n}(\partial Q \backslash \mathscr{J}), \quad u \in X_{(\beta)}^{n+1}(Q),
\end{gathered}
$$

где $X_{(\gamma)}^{r}(Q)=W_{p,(\gamma)}^{r}(Q), \quad Y_{(\beta)}^{n}(\partial Q \backslash \mathscr{J})=W_{p,(\beta)}^{n+1-1 / p}(\partial Q \backslash \mathscr{J})$, если задача рассматривается в весовых пространствах Соболева, и $X_{(\gamma)}^{r}(Q)=H_{\mu,(\gamma)}^{r}(Q)$, $Y_{(\beta)}^{n}(\partial Q \backslash \mathscr{J})=H_{\mu,(\beta)}^{n+1}(\partial Q \backslash \mathscr{J})$ в случае пространств Гёльдера, $n \in \mathbb{N} \cup 0, u, f-$ $2 k$-вектор-функции, $\varphi-k$-вектор-функция. Относительно коэффициентов считаем вьполненными условия $\left.1^{\circ}\right)-5^{\circ}$ ) из $\S 3$. И пусть весовой порядок $\beta$ такой, что имеет место

$$
\beta_{\tau} \notin \mathbb{N} \cup\{0\}, \quad \tau \in \mathscr{J} ;
$$

тогда имеет место 
Tеорема 4.1. Для нётеровости задачи Римана-Гильберта (4.13), (4.14) необходимо и достаточно выполнения условий $\left.\left.1^{\circ}\right)-5^{\circ}\right)$ из $\S 3$ и условия: определитель $\operatorname{det} \mathscr{X}_{j}(\zeta) \neq 0$ на прямой $\operatorname{Re} \zeta=\beta_{\tau_{j}}, \tau_{j}=\tau \in \mathscr{J}$. При выполнении этих условий индекс задачи дается формулой

$$
\text { ind } \mathscr{A}=\operatorname{Ind}_{\Gamma} \bar{\aleph}^{-1} \aleph-\sum_{j=1}^{|\mathscr{F}|} \operatorname{Ind}_{\beta_{j}} \mathscr{X}_{j} y_{j}^{-1}(\zeta)-k(m-1) \text {, }
$$

где $m+1$ - число связных компонент границы $\partial Q, \quad \beta_{j}=\beta_{\tau_{j}},|\mathscr{J}|$ - число точек подмножества $\mathscr{J}$ гранищь, $k$ - половина числа уравнений системы (4.13), матрица $y_{i}(\zeta)$ определена в $\left(4.7^{\prime}\right)$. (Первые слагаемые справа в (4.16) вычисляются по формулам (4.9). По поводу остальных обозначений cм. n. $3 \S 3$.)

Пусть $\beta=\beta_{\tau}$ и $\gamma=\gamma_{\tau}$ - допустимые весовые порядки, т.е. на прямых $\operatorname{Re} \zeta=\beta_{\tau}, \operatorname{Re} \zeta=\gamma_{\tau}, \tau \in \mathscr{J}$, нет нулей $\operatorname{det} \mathscr{X}_{j}(\zeta)$, и пусть выполнены условия $\left.1^{\circ}\right)-5^{\circ}$ ) из $\S 3$. Тогда индексы задачи (4.13), (4.14) связаны равенством

$$
\text { ind } \mathscr{A}_{\gamma}-\text { ind } \mathscr{A}_{\beta}=\sum_{\tau \in \mathscr{J}} S_{\tau}
$$

где $\mathscr{A}_{\gamma}$ - оператор задачи (4.1), (4.2) при $\beta=\gamma, \pm S_{\tau}$ равен числу нулей концевого символа $\mathscr{X}_{j}(\zeta), \tau_{j}=\tau \in \mathscr{J}$ (с учетом кратности) между прямыми $\operatorname{Re} \zeta=\beta_{\tau}, \operatorname{Re} \zeta=\gamma_{\tau}$. Знак "-" соответствует случаю $\beta_{\tau} \leqslant \gamma_{\tau}$, а знак "+"случаю $\beta_{\tau} \geqslant \gamma_{\tau}$.

ДокАЗАТЕЛЬСтво. Пространства $X_{(\gamma)}^{r}(Q), Y_{(\gamma)}^{r}(\partial Q \backslash \mathscr{J})$, как было отмечено в $\S 1$, являются конечномерными расширениями пространств $X_{\gamma}^{r}(Q), Y_{\gamma}^{r}(\partial Q \backslash \mathscr{J})$. Поэтому задачи $(3.1),(3.2)$ и (4.13), (4.14) являются нётеровыми или не являются нётеровыми одновременно. Следовательно, к задаче (4.13), (4.14) применима теорема 3.4. Заменим в (4.13), (4.14) $u$ на $v$ по формуле

$$
u=T \mathscr{S} v, \quad \mathscr{S}=\left(\begin{array}{cc}
\mathbf{1} & i \mathbf{1} \\
\mathbf{1} & -i \mathbf{1}
\end{array}\right)
$$

где 1 - единичная матрица порядка $k, T$ - матрица из разложения (2.1) эллиптической матрицы $\mathbf{a}^{-1} \mathbf{b}$. В результате мы получим задачу, эквивалентную исходной:

$$
\begin{gathered}
\mathscr{D}_{2} v+\mathbb{A}_{1} v=f \in X_{(\beta-1)}^{n}(Q), \\
\Omega v=\varphi \in Y_{(\beta)}^{n}(\partial Q \backslash \mathscr{J}), \quad v \in X_{(\beta)}^{n+1}(Q)
\end{gathered}
$$

(здесь и в дальнейшем мы опускаем младшие члены, они образуют вполне непрерывную добавку к оператору задачи, поэтому не влияют на индекс), где

$$
\begin{aligned}
\mathbb{A} & =\left(\begin{array}{cc}
\operatorname{Re} \Lambda & -\operatorname{Im} \Lambda \\
\operatorname{Im} \Lambda & \operatorname{Re} \Lambda
\end{array}\right), \\
\Omega=G T \mathscr{S} & =2\left(\operatorname{Re}\left(G T_{1}\right),-\operatorname{Im}\left(G T_{1}\right)\right) .
\end{aligned}
$$


Легко видеть, что $T \mathscr{S}$ - вешественная матрища, поэтому $\mathbb{A}$ и $\Omega$ вешественные. Кроме того, они наследуют свойства $\left.1^{\circ}\right)-5^{\circ}$ ) из $\S 3$ исходных матриц (см. ниже).

Вместе с задачей (4.18) будем рассматривать семейство задач, зависяшее от параметра $t \in[0,1]$ :

$$
\begin{gathered}
\mathscr{D}_{2} v+\mathbb{A}(t) \mathscr{D}_{1} v=f \in X_{(\beta-1)}^{n}(Q), \\
\Omega v=\varphi \in Y_{(\beta)}^{n}(\partial Q \backslash \mathscr{J}), \quad v \in X_{(\beta)}^{n+1}(Q),
\end{gathered}
$$

где

$$
\mathbb{A}(t)=(1-t) \mathbb{A}_{0}+t \mathbb{A}, \quad \mathbb{A}_{0}=\left(\begin{array}{cc}
\mathbf{0} & -\mathbf{1} \\
\mathbf{1} & \mathbf{0}
\end{array}\right) .
$$

Заметим, что при $t=1$ задача (4.20) совпадает с (4.18), а при $t=0$ она переходит в задачу для системы Коши-Римана:

$$
\begin{gathered}
\mathscr{D}_{2} v+\mathbb{A}_{0} \mathscr{D}_{1} v=f \in X_{(\beta-1)}^{n}(Q), \\
\Omega v=\varphi \in Y_{(\beta)}^{n}(\partial Q \backslash \mathscr{J}), \quad v \in X_{(\beta)}^{n+1}(Q) .
\end{gathered}
$$

Легко видеть, что (4.20) удовлетворяет условиям $\left.\left.1^{\circ}\right)-5^{\circ}\right)$ из $\S 3$. Проверим сначала условие эллиптичности. Так как матрица при $\mathscr{D}_{1} v$ в (4.20) единичная, для доказательства условия эллиптичности (3.3) достаточно показать, что собственные значения матрицы $\mathbb{A}(t)=\mathbb{A}(t, x)$ имеют отличные от нуля мнимые части. Характеристический многочлен $\Delta(\lambda)=\operatorname{det}(\mathbb{A}(t)-\lambda \mathbf{1})$ представляется в виде произведения:

$$
\Delta(\lambda)=\operatorname{det}(t \Lambda+i(1-t) \mathbf{1}-\lambda \mathbf{1}) \operatorname{det}(t \bar{\Lambda}+i(1-t) \mathbf{1}-\lambda \mathbf{1}) .
$$

Следовательно, собственные значения $\mathbb{A}(t)$ даются равенствами $\lambda(t)=t \lambda+(1-t) i$, $\bar{\lambda}(t)=t \bar{\lambda}-(1-t) i$, где $\lambda$ - любое собственное значение матрицы $\Lambda$ (напомним, что собственные значения матрищ $\Lambda$ и $\bar{\Lambda}$ комплексно сопряжены и имеют отличные от нуля мнимые части). Отсюда следует требуемое.

Для проверки условий дополнительности (3.4), (3.5) заметим, что матрица $\mathbb{A}(t)$ допускает очевидную факторизацию вида (2.1):

$$
\mathbb{A}(t)=\frac{1}{2}\left(\begin{array}{cc}
\mathbf{1} & \mathbf{1} \\
-i \mathbf{1} & i \mathbf{1}
\end{array}\right)\left(\begin{array}{cc}
\Lambda(t) & \mathbf{0} \\
\mathbf{0} & \bar{\Lambda}(t)
\end{array}\right)\left(\begin{array}{cc}
\mathbf{1} & i \mathbf{1} \\
\mathbf{1} & -i \mathbf{1}
\end{array}\right),
$$

где $\Lambda(t)=i t \mathbf{1}+(1-t) \Lambda$. Следовательно, согласно [11] условие дополнительности для (4.20) эквивалентно условию невырожденности на $\partial Q \backslash \mathscr{J}$ следующей матрицы:

$$
\Omega\left(\begin{array}{c}
\mathbf{1} \\
i \mathbf{1}
\end{array}\right)=G \bar{T}_{1}=\aleph
$$

т.е. условию (3.4). Аналогично проверяется и условие (3.5).

Пусть $\mathfrak{A}_{t}^{\tau}(\lambda)$ - соответствующий (4.20) эллиптический пучок (см. (3.7)), и пусть $\mathscr{F}_{\tau}(t)$ - спектр этого пучка. При фиксированном $t \in[0,1]$ в силу теоремы 3.4 задача (4.20) нётеровая, если весовой порядок $\beta=\left(\beta_{\tau}\right)$ такой, что на прямой 
$\operatorname{Re}(i \lambda)=\beta_{\tau}$ нет точек спектра $\mathscr{F}_{\tau}(t)$. Для каждого $t \in[0,1]$ (с учетом (4.15)) фиксируем такой весовой порядок. Так как при малых возмушениях нётеровость и индекс сохраняются, у каждого $t=t_{0} \in[0,1]$ есть окрестность $U_{t_{0}}$ такая, что индекс задачи (4.20) (где $\beta=\beta\left(t_{0}\right)$ ) не меняется в $U_{t_{0}}$. Таким образом, мы имеем покрытие $[0,1]$ интервалами. Выберем из него конечное подпокрытие: $U_{t_{1}}, \ldots, U_{t_{m}}$, $t_{1}=0, t_{m}=1$. Обозначим $\mathscr{A}_{t, \beta}$ оператор, соответствуюший краевой задаче (4.20). Имеем: ind $\mathscr{A}_{t, \beta\left(t_{j}\right)}$ постоянен в $U_{t_{j}}, j=1, \ldots, m$. Пусть $t_{j_{0}}^{0}$ - любая фиксированная точка из пересечения $U_{t_{j}} \cap U_{t_{j+1}}, j=1, \ldots, m$. Согласно формуле (3.7) получим

$$
\text { ind } \mathscr{A}_{t_{j-1}^{0}, \beta\left(t_{j+1}\right)}-\text { ind } \mathscr{A}_{t_{j-1}^{0}, \beta\left(t_{j}\right)}=\sum_{\tau \in \mathscr{J}} S_{\tau, j}
$$

где $S_{\tau, j}$ целое. С учетом этого равенства и постоянства индекса $\mathscr{A}_{t, \beta\left(t_{j}\right)}$ в $U_{t_{j}}$ имеем

$$
\text { ind } \mathscr{A}_{1, \beta(1)}=\text { ind } \mathscr{A}_{0, \beta(0)}+\sum_{\tau \in \mathscr{J}} m_{\tau}
$$

где $m_{\tau}=\sum_{j=1}^{m} S_{\tau, j}$ целое, $\mathscr{A}_{1, \beta(1)}=\mathscr{A}$ - оператор задачи $(4.18)$ с $\beta=\beta(1)$, $\mathscr{A}_{0, \beta(0)}$ - оператор задачи (4.21) для системы Коши-Римана $(\beta=\beta(0))$. Отсюда согласно лемме 4.1 и формуле Солдатова (4.8), примененной к задаче (4.21) для системы Коши-Римана, имеем

$$
\text { ind } \mathscr{A}=\alpha-k(m-1)+\sum_{\tau \in \mathscr{J}} k_{\tau}
$$

где $\alpha$-первое слагаемое из (4.8), $k_{\tau}=m_{\tau}+m_{\tau}^{\prime}, m_{\tau}^{\prime}$ - слагаемое под знаком суммы во втором члене (4.8). (При получении (4.22) учтено замечание перед $5^{\circ}$ ) из $\S 3$ и (4.3), (4.4).) Так как $\beta=\beta(1)$ - произвольный допустимый весовой порядок, формула (4.22) дает нам индекс задачи (4.13), (4.14). Понятно, что формула (4.22) мало пригодна для практических применений. Преобразуем ее в удобную форму.

Пусть $Q_{\tau}$ - односвязная подобласть $Q$ с одной угловой точкой $\tau \in \mathscr{J}$ на границе, причем длина $\partial Q_{\tau}$ равна единице, пересечения $Q_{\tau}, Q$ с некоторой окрестностью $\tau$ совпадают и $\partial Q_{\tau} \backslash\{\tau\}$ принадлежит $C^{\infty}$. Рассмотрим в $Q_{\tau}$ задачу Римана-Гильберта для системы (4.13) с "замороженными" в $\tau$ коэффициентами:

$$
\begin{gathered}
\mathbf{a}(\tau) \mathscr{D}_{1} u+\mathbf{b}(\tau) \mathscr{D}_{2} u=f \in X_{\left(\beta_{\tau}-1\right)}^{n}\left(Q_{\tau}\right), \\
\mathscr{G}_{\tau} u=\varphi \in Y_{\left(\beta_{\tau}\right)}^{n}\left(\partial Q_{\tau} \backslash\{\tau\}\right), \quad u \in X_{\left(\beta_{\tau}\right)}^{n+1}\left(Q_{\tau}\right)
\end{gathered}
$$

$(\tau \in \mathscr{J}),\left\{\beta_{\tau}\right\}=\beta$ - весовой порядок задачи (4.13), (4.14); матрица $\mathscr{G}_{\tau}$ определена равенством $\mathscr{G}_{\tau}=\operatorname{Re}\left((\mathscr{C}, \mathbf{0}) T^{-1}(\tau)\right),(\mathscr{C}, \mathbf{0})-$ блочная матрица, составленная из квадратных матриц $\mathscr{C}, \mathbf{0}$ порядка $k, \mathbf{0}$ - нулевая матрица, $T^{-1}=T^{-1}(\tau)$ - матрица из разложения (2.1) эллиптической матрицы $\mathbf{a}^{-1}(\tau) \mathbf{b}(\tau)$, матрица $\mathscr{C}$ дается равенством

$$
\mathscr{C}=\mathscr{C}(x)=\left(\bar{\aleph}_{-}^{\tau}\right)^{s(x)}\left(\bar{\aleph}_{+}^{\tau}\right)^{1-s(x)},
$$


где $s=s(x)$ - длина дуги границы $\partial Q_{\tau}$ (отсчитываемая от $\tau$ в положительном направлении), $s(\tau+0)=0, s(\tau-0)=1$. Здесь $\aleph_{ \pm}^{\tau}-$ матрицы из $(3.5): \aleph_{ \pm}^{\tau}=$ $G(\tau \pm 0) \bar{T}_{1}(\tau)$. При определении степеней в (4.24) берется непрерьвная ветвь степенной функции.

Понятно, что матрища $\mathscr{G}_{\tau}(x)$ принадлежит $C^{\infty}\left(\partial Q_{\tau} \backslash\{\tau\}\right)$. Кроме того, имеем $\mathscr{G}_{\tau}(x) \bar{T}_{1}(\tau)=\overline{\mathscr{C}}(x)$, где $T_{1}$ - матрица из разложения (2.1). Следовательно, $\operatorname{det} \overline{\mathscr{C}}(x) \neq 0$ всюду на $\partial Q_{\tau}$ (см. $\left.(4.24),(3.4),(3.5)\right), \overline{\mathscr{C}}(\tau \pm 0)=\aleph_{ \pm}^{\tau}$. Значит, условие (4.20) для задач (4.23) и (4.13), (4.14) совпадают. Из последнего следует совпадение спектра $\mathscr{F}_{\tau}$ для (4.23) и (4.13), (4.14), так как спектральной задачей для обеих задач будет (3.7). Подвергнем теперь задачу (4.23) (параллельно с задачей (4.13), (4.14)) тем же преобразованиям (4.17)-(4.20). С учетом предыдущих замечаний видим, что спектр $\mathscr{F} \tau$ для семейства $(4.20)$ и аналогичного семейства для (4.23) совпадают. Поэтому точки $\left(t_{1}, \ldots, t_{m}\right), t_{j}^{0}, j=1, \ldots, m$, окрестности $U_{t_{j}}$ и весовые порядки $\beta=\beta(t)=\left\{\beta_{\tau}(t)\right\}$ в обоих случаях можно взять одинаковыми. Отсюда аналогично (4.22) получим

$$
\text { ind } \mathscr{A}_{\tau}=\alpha_{\tau}+k+k_{\tau}, \quad \tau \in \mathscr{J}
$$

где $k_{\tau}$ точно такое же, как соответствующее слагаемое во второй сумме в (4.22). C другой стороны, согласно лемме 4.1, задача (4.23) тогда и только тогда нётеровая, когда выполнены условия $\left.1^{\circ}\right)-5^{\circ}$ ) и условие

$(\mathrm{S})$ на прямой $\operatorname{Re} \zeta=\beta_{\tau_{j}}, \tau_{j}=\tau \in \mathscr{J}$, нет нулей определителя конщевого символа $x_{j}(\zeta)$.

Таким образом, условия на весовой порядок из теоремы 3.4 и условие (S) эквивалентны. Покажем, что условие (S) эвивалентно условию на концевой символ $\mathscr{X}_{j}(\zeta)$ из теоремы. Действительно, матрица $\Lambda$ из разложения $(2.1)$ и матрица $-J^{-1}$ (см. (4.3), (4.4)), вычисленные в точке $\tau$, подобные: $-J^{-1}=\mathfrak{C} \Lambda \mathfrak{C}^{-1}$. Кроме того, $T_{1}=\mathscr{T}_{1} \mathfrak{C}$. Отсюда легко получим равенство

$$
x_{j}(\zeta)=\overline{\mathfrak{C}} \mathscr{X}_{j}(\zeta) \overline{\mathfrak{C}}^{-1}
$$

из которого следует требуемое.

По формуле Солдатова (4.8) индекс задачи (4.23) дается формулой:

$$
\text { ind } \mathscr{A}_{\tau}=\alpha_{\tau}+k+r_{\tau}
$$

где через $r_{\tau}$ обозначено второе слагаемое в (4.8). При получении (4.27) учтено замечание перед $\left.5^{\circ}\right)$ из $\S 3$. Применение его к системе (4.22) дает соотношение $\mathscr{T}=T \operatorname{diag}\left(\mathfrak{C}^{-1}, \overline{\mathfrak{C}}^{-1}\right)$, где $\mathscr{T}=\left(\mathscr{T}_{1}, \overline{\mathfrak{T}}_{1}\right)$ - матрища из жордановой формы (4.3) (см. (4.4)) матрицы $-\mathbf{b}^{-1}(\tau) \mathbf{a}(\tau), \tau=\tau_{j} \in \mathscr{J}, T=\left(T_{1}, \bar{T}_{1}\right)$ - матрица из (4.17). С учетом этого для матрицы § из (4.7) (естественно, с $\mathscr{G}_{\tau}$ вместо $G$ ) имеем $\aleph=\mathscr{G}_{\tau} \overline{\mathscr{T}}_{1}=\mathscr{G}_{\tau} \bar{T}_{1} \overline{\mathfrak{C}}^{-1}=\overline{\mathscr{C}} \overline{\mathfrak{C}}^{-1}$. Отсюда и из $(4.24)$ получим $\aleph\left(\tau_{j} \pm 0\right)=$ $G\left(\tau_{j} \pm 0\right) \bar{T}_{1} \overline{\mathfrak{C}}^{-1}=G\left(\tau_{j} \pm 0\right) \overline{\mathscr{T}}_{1}$.

Ввиду (4.25), (4.27) имеем $k_{\tau}=r_{\tau}$. Внеся это значение $k_{\tau}$ в $(4.22)$, получим формулу (4.16), где вместо второго слагаемого имеем $\operatorname{Ind}_{\beta_{j}} x_{j}(\zeta) y_{j}^{-1}(\zeta)$. (Здесь 
$x_{j}(\zeta)$ - конщевой символ из (4.7), при нахождении которого нужно учесть, что разложение (4.3) зависит также от $\tau$.) Последнее ввиду (4.26) и свойств Ind [7] легко преобразуется во второе слагаемое (4.16).

Для доказательства второй части теоремы нужно подвергнуть процессу стягивания задачу (4.1), (4.2) одновременно для двух различных допустимых весовых порядков. Тогда с учетом теоремы 3.4 , леммы 4.1 и доказанной части получим требуемое. Теорема доказана.

ЗАмечаниЕ 4.1. Аналогичный теореме 4.1 результат (с той же формулой для индекса) имеет место и для задачи $(4.1),(4.2)$ с $f=0$. Доказывается он аналогично теореме 4.1.

ЗАМЕчАнИЕ 4.2. Как следует из сравнения теоремы 4.1 с теоремами $3.1-3.4$, в последних условие, касаюшееся спектра $\mathscr{F}_{\tau}$, можно заменить на соответствуюшее условие на конщевой символ. При этом предполагаем, что $\beta_{\tau} \neq 0,1, \ldots$

\section{§5. Задача Римана-Гильберта для систем с комплексными коэффициентами}

1. Системы с комплексными коэффициентами. Рассмотрим следующую $\mathbb{R}$-линейную задачу Римана-Гильберта:

$$
\begin{gathered}
\mathscr{L} u \equiv \mathbf{a} \mathscr{D}_{1} u+\mathbf{b} \mathscr{D}_{2} u+\mathbf{c} u=f \in X_{(\beta-1)}^{n}(Q), \\
\operatorname{Re}(G u)=\varphi \in Y_{(\beta)}^{n}(\partial Q \backslash \mathscr{J}), \quad u \in X_{(\beta)}^{n+1}(Q),
\end{gathered}
$$

где $n \in \mathbb{N} \cup\{0\}$, пространства $X_{(\gamma)}^{r}(Q), Y_{(\beta)}^{n}(Q)$ имеют тот же смысл, что и в $(4.13),(4.14)$, причем $X_{(\gamma)}^{r}(Q)$ - пространство комплекснозначных вектор-функций; $u, f, \varphi-k$-вектор-функции.

Для коэффициентов считаем выполненными следующие условия:

1) матрицы $\mathbf{a}, \mathbf{b}, \mathbf{c}-$ квадратные матрицы порядка $k, k \geqslant 1$, причем $\mathbf{a}, \mathbf{b} \in$ $C^{\infty}(\bar{Q}), \mathbf{c} \in C^{\infty}(\bar{Q} \backslash \mathscr{J}) \cap \mathscr{L}_{\infty}(Q)$

2) система (5.1) эллиптическая в $\bar{Q}$ :

$$
\operatorname{det}\left(\mathbf{a}(x) \xi_{1}+\mathbf{b}(x) \xi_{2}\right) \neq 0, \quad x \in \bar{Q}, \quad \xi \in \mathbb{R}^{2} \backslash 0 ;
$$

3) $G \in C^{\infty}(\bar{Q} \backslash \mathscr{J})$ - квадратная матрица порядка $k$, причем она допускает левое и правое продолжения класса $C^{\infty}$ в точки $\mathscr{J}$;

(Ввиду (5.3) матрища $\mathscr{B}=\mathbf{a}^{-1} \mathbf{b}$ эллиптическая, поэтому для нее имеет место разложение (2.1):

$$
\mathscr{B}=T \operatorname{diag}\left(\Lambda_{1}, \Lambda_{2}\right) T^{-1} \text {.) }
$$

4) считаем, что на $\partial Q \backslash \mathscr{J}$ выполнено условие

$$
\operatorname{det} \aleph_{0}(x) \neq 0 \quad \text { всюду на } \quad \partial Q \backslash \mathscr{J},
$$


где $\aleph_{0}=\left(\bar{G} \bar{T}_{1}, G T_{2}\right)$ - блочная квадратная матрица порядка $k$;

5) для предельных значений $\aleph_{ \pm}^{\tau}=\aleph_{0}(\tau \pm 0), \tau \in \mathscr{J}$, матрицы $\aleph_{0}$ в угловых точках справедливы неравенства

$$
\operatorname{det} \aleph_{ \pm}^{\tau} \neq 0, \quad \tau \in \mathscr{J} .
$$

Определим конщевой символ Солдатова для задачи (5.1), (5.2).

Конщевой символ $\mathscr{X}_{j}$ при $\tau_{j}=\tau \in \mathscr{J}$ дается формулой, аналогичной (4.12):

$$
\mathscr{X}_{j}(\zeta)=\mathbf{1}-C^{-1}\left(\tau_{j}-0\right) V_{j}(\zeta) C\left(\tau_{j}+0\right){\overline{V_{j}(\bar{\zeta})}}^{-1},
$$

где при определении $V_{j}(\zeta)$ матрица $\Lambda$ заменена на матрицу $\Lambda=\operatorname{diag}\left(\Lambda_{1}, \bar{\Lambda}_{2}\right)$ :

$$
V_{j}(\zeta)=\left\{\left(\operatorname{Im} q_{k}-\Lambda \operatorname{Re} q_{k}\right)\left(\operatorname{Im} q_{r}-\Lambda \operatorname{Re} q_{r}\right)^{-1}\right\}^{\zeta} \stackrel{\text { def }}{=} \mathfrak{q}^{\zeta}(\Lambda),
$$

где $\Lambda_{1}, \Lambda_{2}$ определены равенством (5.4). А матрица $C$ определена равенствами

$$
C=\bar{\aleph}_{0}^{-1} \aleph_{0}, \quad \aleph_{0}=\left(\bar{G} \bar{T}_{1}, G T_{2}\right) .
$$

Для случаев $k_{ \pm}=0$, где $k_{+}-$число собственных значений матрищы $\mathbf{a}^{-1} \mathbf{b}$ из верхней полуплоскости, а $k_{-}-$из нижней, в формулах (5.6)-(5.8) нужно положить $\aleph_{0}=G, \Lambda=\bar{\Lambda}_{2}=\overline{\mathbf{a}}^{-1} \overline{\mathbf{b}}$ при $k_{+}=0$ и $\aleph_{0}=\bar{G}, \Lambda=\Lambda_{1}=\mathbf{a}^{-1} \mathbf{b}$ при $k_{-}=0$ (см. формулу (2.1)). Следовательно, при $k_{+}=0, k_{-}=k$ концевой символ дается равенством

$$
\begin{gathered}
\mathscr{X}_{j}(\zeta)=\mathbf{1}-C^{-1}\left(\tau_{j}-0\right) V_{j}(\zeta) C\left(\tau_{j}+0\right){\overline{V_{j}(\bar{\zeta})}}^{-1}, \\
V_{j}(\zeta)=\mathfrak{q}^{\zeta}\left(\overline{\mathbf{a}}^{-1} \overline{\mathbf{b}}\right), \quad C=\bar{G}^{-1} G,
\end{gathered}
$$

а при $k_{-}=0, k_{+}=k$ концевой символ дается равенством

$$
\begin{gathered}
\mathscr{X}_{j}(\zeta)=\mathbf{1}-C^{-1}\left(\tau_{j}-0\right) v_{j}(\zeta) C\left(\tau_{j}+0\right){\overline{v_{j}(\bar{\zeta})}}^{-1} \\
V_{j}(\zeta)=\mathfrak{q}^{\zeta}\left(\mathbf{a}^{-1} \mathbf{b}\right), \quad C=G^{-1} \bar{G} .
\end{gathered}
$$

Справедлива ${ }^{1}$

Теорема 5.1. Для нётеровости задачи Римана-Гильберта (5.1), (5.2) необходимо и достаточно выполнения условий 1)-5) и условия: $\operatorname{det} \mathscr{X}_{j}(\zeta) \neq 0$ на прямой $\operatorname{Re} \zeta=\beta_{\tau_{j}}, \quad \tau_{j}=\tau \in \mathscr{J}$. При выполнении этих условий индекс задачи дается формулой

$$
\text { ind } \mathscr{A}=\operatorname{Ind}_{\Gamma} \bar{\aleph}_{0}^{-1} \aleph_{0}-\sum_{j=1}^{|\mathscr{J}|} \operatorname{Ind}_{\beta_{j}} \mathscr{X}_{j}(\zeta) y_{j}^{-1}(\zeta)-k(m-1)
$$

\footnotetext{
${ }^{1}$ Всюду в параграфе весовой порядок $\beta$ удовлетворяет условию (4.15), отмеченному перед теоремой 4.1.
} 
где $\aleph_{0}=\left(\bar{G} \bar{T}_{1}, G T_{2}\right)$, m, $y_{j}$ такие же, как в теореме 4.1.

Пусть $\beta=\left(\beta_{\tau}\right)$ и $\gamma=\left(\gamma_{\tau}\right)$ - допустимые весовые порядки, т.е. на прямых $\operatorname{Re} \zeta=\beta_{\tau}, \operatorname{Re} \zeta=\gamma_{\tau}, \tau \in \mathscr{J}$, нет нулей определителя концевого символа, и пусть выполнены условия 1)-5). Тогда индексы задачи (5.1), (5.2) связаны равенством

$$
\text { ind } \mathscr{A}_{\gamma}-\text { ind } \mathscr{A}_{\beta}=\sum_{\tau \in \mathscr{J}} S_{\tau}
$$

где $\mathscr{A}_{\gamma}$ - оператор задачи (5.1), (5.2) при $\beta=\gamma, \pm S_{\tau}$ равен числу нулей концевого символа $\mathscr{X}_{j}(\zeta), \tau_{j}=\tau \in \mathscr{J}$ (с учетом кратности) между прямыми $\operatorname{Re} \zeta=\beta_{\tau}, \operatorname{Re} \zeta=\gamma_{\tau}$. Знак "-" соответствует случаю $\beta_{\tau} \leqslant \gamma_{\tau}$, а знак "+"случаю $\beta_{\tau} \geqslant \gamma_{\tau}$.

ДокАЗАТЕЛЬСТво. Пусть $\mathbf{a}^{-1} \mathbf{b}=E+i F, u=u_{1}+u_{2}, \mathbf{a}^{-1} f=f_{1}+f_{2}$, $G=G_{1}+i G_{2}, \mathbf{a}^{-1} \mathbf{c}=c_{1}+i c_{2}$. Тогда задача (5.1), (5.2) эквивалентна следуюшей краевой задаче для эллиптической системы с действительными коэффициентами:

$$
\begin{gathered}
\frac{\partial v}{\partial x_{1}}+\mathfrak{B} \frac{\partial v}{\partial x_{2}}+\mathfrak{C} v=g \in X_{(\beta-1)}^{n}(Q), \quad \mathfrak{B}=\left(\begin{array}{rr}
E & -F \\
F & E
\end{array}\right), \\
G_{0} v=\varphi \in Y_{(\beta)}^{n}(\partial Q \backslash \mathscr{J}), \quad v \in X_{(\beta)}^{n+1}(Q),
\end{gathered}
$$

где $v=\left(v_{1}, v_{2}\right), g=\left(f_{1}, f_{2}\right)$ - вектор-столбцы, $G_{0}=\left(G_{1}, G_{2}\right)$, матрица $\mathfrak{C}$ имеет блочную структуру, аналогичную $\mathfrak{B}$.

Следовательно, задачи (5.1), (5.2) и (5.10) являются нётеровыми или не являются нётеровыми одновременно и в случае нётеровости имеют равные индексы. Согласно теореме 4.1 для нётеровости задачи (5.10) необходимо и достаточно выполнения условий п. $1 \S 4$ и условия $\operatorname{det} \widetilde{\mathscr{X}}_{j}(\zeta) \neq 0$ на прямой $\operatorname{Re} \zeta=\beta_{\tau_{j}}$ (где через $\widetilde{\mathscr{X}}_{j}$ обозначен концевой символ задачи $(5.10)$ из теоремы 4.1$)$ и при выполнении последних индекс задачи дается равенством (4.16). Значит, для доказательства теоремы достаточно показать эквивалентность отмеченных условий и условий теоремы и то, что формула индекса (4.16) преобразуется в (5.9). Остановимся подробнее на последнем. Легко видеть (см. [12]), что матрица $\mathfrak{B}$ представляется в виде произведения:

$$
\begin{gathered}
\mathfrak{B}=T \operatorname{diag}\left(\Lambda_{1}, \bar{\Lambda}_{2}, \bar{\Lambda}_{1}, \Lambda_{2}\right) T^{-1}, \\
T=(\mathfrak{X}, \overline{\mathfrak{X}}), \quad \mathfrak{X}=\left(\begin{array}{cc}
T_{1} & \bar{T}_{2} \\
-i T_{1} & i \bar{T}_{2}
\end{array}\right),
\end{gathered}
$$

где $T_{1}, T_{2}, \Lambda_{1}, \Lambda_{2}$ - матрищы из разложения (2.1) эллиптической матрицы $\mathbf{a}^{-1} \mathbf{b}$. Понятно, что $(*)$ является одним из разложений $(2.1)$ эллиптической матрицы $\mathfrak{B}$. С учетом этого имеем $\aleph=G_{0} \overline{\mathfrak{X}}=\left(\bar{G}_{1}, G T_{2}\right)=\aleph_{0}$, откуда следует требуемое. Теорема доказана. 
ЗАмечаниЕ 5.1. Для задачи (5.1), (5.2) с однородной системой $(f=0)$ имеет место утверждение, аналогичное теореме 5.1 (при этом формулы такие же, как в теореме 5.1). Оно доказывается аналогично с привлечением замечания 4.1.

В некоторых случаях формула (5.9) для индекса упрошается, а именно, имеет место

СЛЕДСТВИЕ 5.1. Предположим, что все корни характеристического полинома $\Delta(\lambda)=\operatorname{det}\left(\lambda-\mathbf{a}^{-1} \mathbf{b}\right)$ расположсены или в верхней полуплоскости, или в нижней. Тогда в формуле для индекса (5.9) $\aleph_{0}=\bar{G}$, а концевой символ дается равенством (5.6") в первом случае и $\aleph_{0}=G$, а концевой символ равенством (5.6') во втором.

ДокАЗАТЕльСТво. В первом случае в качестве разложения (2.1) выступает тривиальное разложение $\mathbf{a}^{-1} \mathbf{b}=T\left(\mathbf{a}^{-1} \mathbf{b}\right) T^{-1}, T=T_{1}=\mathbf{1}$, во втором аналогично $T=T_{2}=\mathbf{1}$. Отсюда с учетом формулы $(5.9)$ и замечаний перед теоремой о концевом символе получим требуемое. Следствие доказано.

2. Правильно эллиптические системы. Рассмотрим следующую задачу:

$$
\begin{aligned}
& \mathscr{L} u \equiv \mathbf{a} \mathscr{D}_{1} u+\mathbf{b} \mathscr{D}_{2} u+\mathbf{c} u=f \in X_{(\beta-1)}^{n}(Q), \\
& G_{0} u=\varphi \in Y_{(\beta)}^{n}(\partial Q \backslash \mathscr{J}), \quad u \in X_{(\beta)}^{n+1}(Q),
\end{aligned}
$$

где $\mathbf{a}, \mathbf{b}, \mathbf{c}-$ квадратные матрицы порядка $2 k, G_{0}-$ прямоугольная $(k \times 2 k)$-матрища. В отличие от задачи (4.1), (4.2) мы здесь допускаем комплексные значения элементов.

Предположим, что система (5.11) правильно эллиптическая, т.е. корни характеристического полинома $\operatorname{det}\left(\lambda-\mathbf{a}^{-1} \mathbf{b}\right)$ (с учетом кратностей) расположены поровну в верхней и нижней полуплоскостях. Предполагаем также, что для (5.11), $(5.12)$ выполнены условия 1)-5) с заменой $G$ на $G_{0}$ и $k$ на $2 k$.

Концевой символ $\widetilde{\mathscr{X}}_{j}(\zeta)$ при $\tau_{j}=\tau \in \mathscr{J}$ дается формулами

$$
\begin{aligned}
\widetilde{\mathscr{X}}_{j}=\operatorname{diag}\left(\mathscr{X}_{1 j}(\zeta), \mathscr{X}_{2 j}(\zeta)\right), \\
\mathscr{X}_{1 j}(\zeta)=\mathbf{1}-C^{-1}\left(\tau_{j}-0\right) V_{2 j}(\zeta) C\left(\tau_{j}+0\right) \overline{V_{1 j}(\bar{\zeta})}-1 \\
\mathscr{X}_{2 j}(\zeta)=\mathbf{1}-\overline{C\left(\tau_{j}-0\right)} V_{1 j}(\zeta) \overline{C^{-1}\left(\tau_{j}+0\right)} \overline{V_{2 j}(\bar{\zeta})}-1 \\
V_{1 j}(\zeta)=\left\{\left(\operatorname{Im} q_{k}-\Lambda_{1} \operatorname{Re} q_{k}\right)\left(\operatorname{Im} q_{r}-\Lambda_{1} \operatorname{Re} q_{r}\right)^{-1}\right\}^{\zeta}, \\
V_{2 j}(\zeta)=\left\{\left(\operatorname{Im} q_{k}-\bar{\Lambda}_{2} \operatorname{Re} q_{k}\right)\left(\operatorname{Im} q_{r}-\bar{\Lambda}_{2} \operatorname{Re} q_{r}\right)^{-1}\right\}^{\zeta}, \\
C=\bar{\aleph}_{2}^{-1} \aleph_{1}, \quad \aleph_{1}=\bar{G}_{0} \bar{T}_{1}, \quad \aleph_{2}=G_{0} T_{2} .
\end{aligned}
$$

Имеет место 
ТЕОРЕМА 5.2. Условия 1)-5) (включая правильную әллиптичность) и условие: $\operatorname{det} \mathscr{X}_{j}(\zeta) \neq 0$ на прямой $\operatorname{Re} \zeta=\beta_{\tau}, \tau=\tau_{j} \in \mathscr{J}$, необходимы и достаточны для нётеровости задачи (5.11), (5.12). При выполнении этих условий индекс задачи дается равенством

$$
\text { ind } \mathscr{A}=2^{-1} \sum_{l=1}^{2}\left(\operatorname{Ind}_{\Gamma} \bar{\aleph}_{l}^{-1} \aleph_{l}-\sum_{j=1}^{|\mathscr{F}|} \operatorname{Ind}_{\beta_{j}} \mathscr{X}_{l j}(\zeta) y_{l j}^{-1}(\zeta)\right)-k(m-1) \text {, }
$$

где $\aleph_{1}=\bar{G}_{0} \bar{T}_{1}, \aleph_{2}=G_{0} T_{2}, \quad m, \beta_{j}$ такие же, как в теореме 5.1. Матрица $y_{l j}(\zeta), l=1,2$, определяется аналогично $y_{j}(\zeta)$ из теоремы 4.1 , вместо $v_{j}(\zeta)$ берем матрицу $v_{l j}(\zeta), \quad l=1,2$.

Теорема дополняется и второй частью, аналогичной теореме 5.1.

ДокАЗАТЕльство. Задача (5.11), (5.12) $\mathbb{C}$-линейная. Перейдем от нее к $\mathbb{R}$-линейной задаче $(5.1),(5.2)$ для системы $2 k$ уравнений, для чего граничные условия (5.12) достаточно представить в виде $\operatorname{Re}\left(G_{0} u\right)=\varphi_{1}, \operatorname{Re}\left(-i G_{0} u\right)=\varphi_{2}$, где $\varphi_{1}+i \varphi_{2}=\varphi$. При этом обе задачи эквивалентны в смысле нётеровости, и в случае нётеровости индекс второй задачи в два раза больше первой. Матрица $G$ из (5.2) теперь имеет блочно диагональную структуру, $G=\operatorname{diag}\left(G_{0},-i G_{0}\right)$. Матрища $\aleph_{0}$ из теоремы 5.1 здесь следующего вида:

$$
\aleph_{0}=\left(\begin{array}{cc}
\bar{G}_{0} \bar{T}_{1} & G_{0} T_{2} \\
i \bar{G}_{0} \bar{T}_{1} & -i G_{0} T_{2}
\end{array}\right)
$$

где $T_{0}=\left(T_{1}, T_{2}\right)$ - матрица из разложения (2.1) эллиптической матрицы $\mathbf{a}^{-1} \mathbf{b}$ порядка $2 k$. Заметим, что $T_{1}$ и $T_{2}-(2 k \times k)$-матрицы ввиду правильной эллиптичности системы. С учетом этих замечаний достаточно показать, что формула (5.9) (где вместо $k$ имеем $2 k$ ) после деления на 2 переходит в формулу (5.14). Очевидно, что det $\aleph_{0}=(-2 i)^{k} \operatorname{det} \aleph_{1} \operatorname{det} \aleph_{2}$, поэтому первое слагаемое в (5.9) преобразуется в первое слагаемое из (5.14). Рассмотрим теперь второе слагаемое. Для матриц $\aleph$ и $C$ из (5.6)-(5.8) легко получим

$$
\begin{gathered}
\aleph=\left(\bar{G} \bar{T}_{1}, G T_{1}\right)=\left(\begin{array}{cc}
\aleph_{1} & \aleph_{2} \\
i \aleph_{1} & -i \aleph_{2}
\end{array}\right), \\
\bar{C}=C^{-1}=\left(\begin{array}{cc}
0 & \bar{\aleph}_{1}^{-1} \aleph_{2} \\
\bar{\aleph}_{2}^{-1} \aleph_{1} & 0
\end{array}\right) .
\end{gathered}
$$

Отсюда для концевого символа (5.6) имеем

$$
\begin{array}{r}
\mathscr{X}_{j}(\zeta)=\operatorname{diag}\left(\mathbf{1}-\aleph_{1 k}^{-1} \bar{\aleph}_{2 k} V_{2 j}(\zeta) \bar{\aleph}_{2 r}^{-1} \aleph_{1 r}{\overline{V_{1 j}(\bar{\zeta})}}^{-1},\right. \\
\left.1-\aleph_{2 k}^{-1} \bar{\aleph}_{1 k} V_{1 j}(\zeta) \bar{\aleph}_{1 r}^{-1} \aleph_{2 r}{\overline{V_{2 j}(\bar{\zeta})}}^{-1}\right), \\
\mathfrak{D}_{k}=\mathfrak{D}\left(\tau_{j}-0\right), \quad \mathfrak{D}_{r}=\mathfrak{D}\left(\tau_{j}+0\right), \quad \mathfrak{D}=\aleph_{1}, \aleph_{2} .
\end{array}
$$

Это выражение и есть конщевой символ рассматриваемой задачи. Теорема доказана. 
ЗАмечаниЕ 5.2. Для задачи $(5.11),(5.12)$ с однородной системой $(f=0)$ имеет место утверждение, аналогичное теореме 5.2 (при этом формулы такие же, как в теореме 5.1). Оно доказывается аналогично с привлечением замечания 5.1.

Рассмотрим один пример, который понадобится в дальнейшем.

ПримеР 5.1. Следуюшая задача Римана-Гильберта для системы Коши-Римана:

$$
\begin{gathered}
\frac{\partial u}{\partial x_{1}}+i \frac{\partial u}{\partial x_{2}}=f \in X_{(\beta-1)}^{n-1}(Q), \\
\operatorname{Re} u=\varphi \in Y_{(\beta)}^{n}(\partial Q \backslash \mathscr{J}), \quad u \in X_{(\beta)}^{n}(Q),
\end{gathered}
$$

нётеровая лишь тогда, когда $\beta_{\tau} \neq \pi k / \vartheta_{\tau}, k \in \mathbb{Z}, \tau \in \mathscr{J}$, где $\mathbb{Z}$ - множество целых чисел. При выполнении последних индекс задачи дается формулой

$$
\text { ind } \bar{\partial}=k \sum_{\tau \in \mathscr{J}}\left(\left[\beta_{\tau} \frac{\vartheta_{\tau}}{\pi}\right]+1\right)-k(m-1) \text {, }
$$

где $k$ - число уравнений системы, $[a]$ - целая часть числа $a, m+1$ - порядок связности области.

Действительно, задача (5.15) есть частный случай задачи из следствия 5.1, $G=\mathbf{1}$ - единичная матрица порядка $k$. Следовательно, для нахождения условий нётеровости нужно вычислить концевой символ. Так как (5.15) распадается на $k$ одинаковых задач, для компонент вектора $u$ достаточно ограничиться случаем $k=1$. Конщевой символ здесь определяется просто:

$$
V_{j}(\zeta)=v_{j}(\zeta)=e^{i \vartheta_{\tau} \zeta}, \quad \mathscr{X}_{j}(\zeta)=1-e^{2 i \vartheta_{\tau} \zeta}, \quad \tau=\tau_{j} \in \mathscr{J},
$$

где $\vartheta_{\tau}$ - раствор криволинейного сектора $Q_{\tau} \subset Q$ с вершиной в точке $\tau \in \mathscr{J}$. Значит, $\zeta=\pi k / \vartheta_{\tau}-$ корни концевого символа. Ввиду следствия 5.1 критерием нётеровости (5.15) является условие $\beta_{\tau} \neq \pi k / \vartheta_{\tau}$. Вычислим теперь индекс задачи. Первое слагаемое (5.9) ввиду следствия 5.1 и $G=\mathbf{1}$ равно нулю. Второе слагаемое при $\beta_{j}=\beta_{\tau}=-1 / 4, \tau=\tau_{j} \in \mathscr{J}$, также равно нулю. (Заметим, что корни концевого символа расположены вне полосы $-1 / 2 \leqslant \operatorname{Re} \zeta<0$.) Отсюда с учетом второй части теоремы 5.1 получим (5.16).

\section{§6. Краевые задачи для систем Дуглиса-Ниренберга. О нётеровости задачи}

1. Напомним понятие эллиптичности по Дуглису-Ниренбергу. В ограниченной области $Q$ плоскости рассмотрим систему

$$
\mathscr{L}(x, \mathscr{D}) v=f,
$$

где $\mathscr{L}=\mathscr{L}(x, \mathscr{D})=\left\{a_{i j}(x, \mathscr{D})\right\}$ - квадратная матрица порядка $\ell$, элементы которой - линейные дифференциальные выражения. Коэффициенты $a_{i j}(x, \mathscr{D})$ (как дифференциального выражения) - непрерьвные в $\bar{Q}$ функции. 
Матрище $\mathscr{L}$ поставим в соответствие матрищу $\mathscr{L}(x, \xi)=\left\{a_{i j}(x, \xi)\right\}$ с полиномиальньги по $\xi_{1}$ и $\xi_{2},\left(\xi_{1}, \xi_{2}\right)=\xi$, элементами. Говорят, что система (6.1) әллиптическая по Дуглису-Ниренбергу в области $\bar{Q}$, если найдутся целые числа $s_{i}$ и $t_{i}$, $i=1, \ldots, \ell$, удовлетворяющие следуюшим условиям:

1) сумма $N=\sum_{i=1}^{\ell}\left(s_{i}+t_{i}\right)$ (для всех $x \in \bar{Q}$ ) равна степени полинома $L(x, \xi)=$ $\operatorname{det} \mathscr{L}(x, \xi)$, т.е. $N=\operatorname{ord} L$, и $N=2 N^{\prime}$ - четное число;

2 ) сумма $s_{i}+t_{j}$ не меньше степени $a_{i j}(x, \xi)$, причем, если $s_{i}+t_{j}<0$, считаем, что $a_{i j}=0$;

$3)$ пусть $\mathscr{L}_{0}(x, \xi)$ - главная часть $\mathscr{L}(x, \xi)$, каждый элемент которой есть главная однородная часть $a_{i j}(x, \xi)$, состояшая из слагаемых степени $s_{i}+t_{j}$; тогда

$$
\operatorname{det} \mathscr{L}_{0}(x, \xi) \neq 0 \quad \forall x \in \bar{Q}, \quad \xi \in \mathbb{R}^{2} \backslash 0
$$

(условие әллиптичности);

$4)$ все корни полинома $P(\lambda)=\operatorname{det} \mathscr{L}_{0}(x, \xi), \xi=(-\lambda, 1), x \in \bar{Q}$ (с учетом кратностей), расположены поровну в верхней $\operatorname{Im} \lambda>0$ и нижней $\operatorname{Im} \lambda<0$ полуплоскостях (правильная әллиптичность).

В случае системы с действительными коэффициентами условие 4) и предположение о четности $N$ из 1$)$ лишние. Они являются следствиями условия эллиптичности (6.2).

Набор чисел $s_{i}, t_{i}$, участвующий в определении эллиптичности, определен неоднозначно. Каждый такой набор можно заменить на $s_{i}-a, t_{i}+a$, где $a-$ любое целое. Более того, в предположении, что матрица $\mathscr{L}_{0}$ имеет клеточно-диагональную структуру, $\mathscr{L}_{0}=\operatorname{diag}\left(\mathscr{L}_{1}, \ldots, \mathscr{L}_{q}\right), 1 \leqslant q \leqslant \ell$, с квадратными блоками $\mathscr{L}_{i}$ на диагонали, в пределах каждого блока от $s_{i}$ можно отнять, а к $t_{i}$ прибавить одно и то же число (любое). В дальнейшем эти целые числа будем считать такими, что для каждого блока $\max s_{i}=0$. При такой нормировке набор определен однозначно.

Система (6.1), для которой все $s_{i}$ равны нулю, называется системой Петров$c \kappa о г о$. Система Петровского, для которой все $t_{i}$ равны $r$, называется однородной системой порядка $r$.

Для системы (6.1) рассматривается следуюшая краевая задача:

$$
\begin{aligned}
& \mathscr{L}(x, \mathscr{D}) v=f \in X_{\beta-a}^{n}(Q), \\
& \mathscr{B}(x, \mathscr{D}) v=\varphi \in Y_{\beta-b}^{n}(\partial Q \backslash \mathscr{J}), \quad v \in X_{\beta}^{n+1}(Q),
\end{aligned}
$$

где $\mathscr{B}(x, \mathscr{D})=b_{i j}(x, \mathscr{D})$ - прямоугольная $\left(N^{\prime} \times \ell\right)$-матрица, элементы которой линейные дифференциальные выражения с коэффициентами из $C^{\infty}(\partial Q \backslash \mathscr{J})$.

Пусть $\Gamma_{j}, j=1, \ldots,|\mathscr{J}|,-$ гладкие дуги, составлющие $\partial Q$.

5) Предполагаем, что найдутся целые числа $\sigma_{1 l}, \ldots, \sigma_{N^{\prime} l}$ такие, что сумма $\sigma_{i l}+t_{j}$ равна степени ord $b_{i j}$ на $\Gamma_{l}, l=1, \ldots,|\mathscr{J}|$, причем при $\sigma_{i l}+t_{j}<0$ имеем $b_{i j}=0$.

Пусть область $Q$ такая же, как в п. $1 \S 3$. В дальнейшем коэффициенты уравнений и краевых условий такие, что они принадлежат $C^{\infty}(\bar{Q}), C^{\infty}(\partial Q \backslash \mathscr{J})$ соответственно, причем коэффициенты краевых условий допускают гладкие (класса $C^{\infty}$ ) 
левые и правые продолжения по граничным дугам в угловые точки $\mathscr{J}$. Кроме того, для них выполнены условия Шапиро-Лопатинского.

Напомним последнее. Пусть $\nu=\left(\nu_{1}, \nu_{2}\right)$ - нормаль к границе $Q$ в точке $x \in$ $\partial Q \backslash \mathscr{J}$ и $\xi$-вектор касательной в $x$. Пусть $\mathscr{L}_{0}$ и $\mathscr{B}_{0}-$ главные части $\mathscr{L}$ и $\mathscr{B}$ (получаемые из них вычеркиванием слагаемых степеней меньше ord $a_{i j}$ и ord $b_{i j}$ из $a_{i j}$ и $b_{i j}$ соответственно). Говорят, что краевая задача удовлетворяет условию допольнительности (условию Шапиро-Лопатинского), если строки матрищы

$$
C(\tau)=\mathscr{B}_{0}(x, \xi+\tau \nu) \mathscr{L}_{0}^{v}(x, \xi+\tau \nu)
$$

в каждой точке $x \in \partial Q \backslash \mathscr{J}$ линейно независимы по модулю многочлена

$$
M^{+}(\tau)=\prod_{j=1}^{N^{\prime}}\left(\tau-\tau_{j}^{+}\right),
$$

где $\tau_{j}^{+}$- корни многочлена $\operatorname{det} \mathscr{L}_{0}(x, \xi+\tau \nu)$ с $\operatorname{Im} \tau>0$. Здесь $\mathscr{L}_{0}^{v}$ - взаимная (или присоединенная) матрища для $\mathscr{L}_{0}$, т.е. матрица, полученная транспонированием матрицы из алгебраических дополнений элементов $\mathscr{L}_{0}$.

6) Предполагаем, что условие дополнительности выполнено в каждой точке $\partial Q \backslash \mathscr{J}$. Кроме того, это же условие выполнено для продолжений матрищы $\mathscr{B}_{0}$ в угловые точки $\tau \in \mathscr{J}$.

Пространства $X_{\beta-a}^{n}(Q), Y_{\beta-b}^{n}(\partial Q \backslash \mathscr{J}), X_{\beta}^{n}(Q)$, когда задача рассматривается над весовыми пространствами Соболева, определены равенствами

$$
\begin{gathered}
X_{\beta}^{n}(Q)=\prod_{i=1}^{\ell} W_{p, \beta+t_{i}-t_{0}}^{n+t_{i}}(Q), \quad X_{\beta-a}^{n}(Q)=\prod_{i=1}^{\ell} W_{p, \beta-s_{i}-t_{0}}^{n-s_{i}}(Q), \\
Y_{\beta-b}^{n}(\partial Q \backslash \mathscr{J})=\prod_{i=1}^{N / 2} W_{p, \beta-\sigma_{i}-t_{0}}^{n-\sigma_{i}-1 / p}(\partial Q \backslash \mathscr{J}),
\end{gathered}
$$

а когда задача рассматривается над пространствами Гёльдера, они даются равенствами

$$
\begin{gathered}
X_{\beta}^{n}(Q)=\prod_{i=1}^{\ell} H_{\mu, \beta+t_{i}-t_{0}}^{n+t_{i}}(Q), \quad X_{\beta-a}^{n}(Q)=\prod_{i=1}^{\ell} H_{\mu, \beta-s_{i}-t_{0}}^{n-s_{i}}(Q), \\
Y_{\beta-b}^{n}(\partial Q \backslash \mathscr{J})=\prod_{i=1}^{N / 2} H_{\mu, \beta-\sigma_{i}-t_{0}}^{n-\sigma_{i}}(\partial Q \backslash \mathscr{J}),
\end{gathered}
$$

где $t_{0}=\max _{j}$. Кроме того, $n$ в (6.4) - любое фиксированное неотрицательное целое такое, что $n>\sigma_{0}=\max \left\{\sigma_{i} \mid i=1, \ldots, N^{\prime}\right\}$, а в (6.5) такое, что $n \geqslant \max \left\{0, \sigma_{0}\right\}$. 
2. Вопрос о нётеровости задачи (6.3) изучим по схеме, предложенной в § 3 . Сначала рассмотрим задачу в угле:

$$
\begin{aligned}
& \widetilde{\mathscr{L}}_{0}(x, \mathscr{D}) v=f \in X_{\beta-a}^{n}\left(K^{\tau}\right), \\
& \widetilde{\mathscr{B}}_{0}(x, \mathscr{D}) v=\varphi \in Y_{\beta-b}^{n}\left(\partial K^{\tau} \backslash \tau\right), \quad v \in X_{\beta}^{n}\left(K^{\tau}\right) .
\end{aligned}
$$

(Поясним переход от $(6.3)$ к (6.6). Оставим в (6.3) главные части $\mathscr{L}_{0}, \mathscr{B}_{0}$, затем перейдем к новой системе координат посредством диффеоморфизма из п. $1 \S 3$. Полученные в результате операторы и обозначены через $\widetilde{\mathscr{L}}_{0}, \widetilde{\mathscr{B}}_{0}$.)

Переходим в (6.6) к полярной системе координат $(r, \vartheta)$ с центром в точке $\tau$ и осью, направленной по выходящей стороне. Тогда после несложных преобразований (см. [10, с. 84]) получим, что (6.6) представляется в виде

$$
\begin{aligned}
& \sum_{j=1}^{\ell} \mathbb{L}_{h j}\left(\vartheta, D_{\vartheta}, r D_{r}-i t_{j}\right) r^{-i t_{j}} v_{j}(r, \vartheta)=r^{s_{h}} f_{h}(r, \vartheta), \\
& \sum_{j=1}^{N / 2} \mathbb{B}_{q j}\left(\vartheta, D_{\vartheta}, r D_{r}-i t_{j}\right) r^{-i t_{j}} v_{j}(r, \vartheta)=r^{\sigma_{q}} \varphi_{h}(r, \vartheta),
\end{aligned}
$$

$h=1, \ldots, \ell, q=1, \ldots, N / 2$, где $D_{\vartheta}=-i \partial / \partial \vartheta, D_{r}=-i \partial / \partial r$. Подействуем на (6.7), (6.8) преобразованием Меллина по переменной $r$. В результате мы получим эллиптический пучок $[10$, c. $84-85]$ :

$$
\mathbb{C} \ni \lambda \mapsto \widetilde{\mathscr{U}}^{\tau}(\lambda)=\left\{\mathbb{L}^{\tau}(\lambda), \mathbb{B}^{\tau}(\lambda)\right\},
$$

где матрицы $\mathbb{L}^{\tau}(\lambda), \mathbb{B}^{\tau}(\lambda)$ определяются равенствами

$$
\begin{aligned}
& \mathbb{L}^{\tau}(\lambda)=\left\{\mathbb{L}_{h j}\left(\vartheta, D_{\vartheta}, \lambda-i t_{j}\right) \mid h, j=1, \ldots, \ell\right\} \\
& \mathbb{B}^{\tau}(\lambda)=\left\{\mathbb{B}_{q j}\left(\vartheta, D_{\vartheta}, \lambda-i t_{j}\right) \mid q=1, \ldots, N / 2, j=1, \ldots, \ell\right\} .
\end{aligned}
$$

Спектр пучка (6.9) обладает свойствами пучка из §3. Имеет место

ЛЕмма 6.1. Задача (6.6) однозначно разрешима лишь тогда, когда на прямой $\operatorname{Re}(i \lambda)=\beta_{\tau}$ нет точек спектра пучка $(6.9)$.

ДокаЗАТЕЛЬСТво см. [10, с. 85, 103-104]. Разница в формулировках, приведенных в [10], и леммы 6.1 пояснялась в п. $1 \S 3$.

Пусть $\mathscr{U}^{\tau}(\lambda)$ - пучок, полученньй из $\widetilde{\mathscr{U}}^{\tau}(\lambda)$ заменой $\widetilde{\mathscr{L}}_{0}(x, \mathscr{D})$ на $\mathscr{L}_{0}(\tau, \mathscr{D})$ и $\widetilde{\mathscr{B}}_{0}(x, \mathscr{D})$ на $\mathscr{B}_{0}^{\tau}(\mathscr{D})$, где $\mathscr{B}_{0}^{\tau}(\xi)=\mathscr{B}_{0}(\tau-0, \xi)$ на "входящей" стороне и $\mathscr{B}_{0}^{\tau}(\xi)=$ $\mathscr{B}_{0}(\tau+0, \xi)$ на "выходящей" . ("Входяшая" и "выходящая" стороны понимаются в смысле обхода границы в положительную сторону, оставляющей область справа.)

Имеет место 
УТВЕРЖДЕНИЕ 6.1. Для справедливости априорной оченки

$$
c\left\|v ; X_{\beta}^{n}(Q)\right\| \leqslant\left\|\mathscr{L} v ; X_{\beta-a}^{n}(Q)\right\|+\left\|\mathscr{B} v ; Y_{\beta-b}^{n}(\partial Q \backslash \mathscr{J})\right\|+\left\|v ; X_{\beta}^{n-1}(Q)\right\|,
$$

әде с >0 - константа, не зависящая от $v \in X_{\beta}^{n}(Q)$, для задачи (6.3) необходимо и достаточно выполнения условий 1$)-6)$ и условия: на прямой $\operatorname{Re}(i \lambda)=\beta_{\tau}$ нет точек спектра пучка $\mathscr{U}^{\tau}(\lambda), \tau \in \mathscr{J}$.

ДокАЗАТЕЛЬСТво. Действительно, пусть $\zeta_{0}, \ldots, \zeta_{\tau}$ - разбиение единицы из определений норм (1.8) и (1.16). Из внутренних оценок Шаудеровского типа [17] следует, что

$$
\begin{aligned}
c_{1}\left\|\zeta_{0} v ; X_{\beta}^{n}(Q)\right\| \leqslant & \left\|\zeta_{0} \mathscr{L} v ; X_{\beta-a}^{n}(Q)\right\|+\left\|\zeta_{0} \mathscr{B} v ; Y_{\beta-b}^{n}(\partial Q \backslash \mathscr{J})\right\| \\
& +\left\|v ; X_{\beta}^{n-1}(Q)\right\|,
\end{aligned}
$$

где $c_{1}>0$ - константа. Теперь получим аналогичные оценки вблизи угловых точек $\tau \in \mathscr{J}$. Вблизи $\tau$ имеем

$$
\begin{aligned}
\mathscr{L}_{0}(\tau, \mathscr{D})\left(\zeta_{\tau} v\right) & =F, \\
\mathscr{B}_{0}^{\tau}(\mathscr{D})\left(\zeta_{\tau} v\right) & =\Phi,
\end{aligned}
$$

где

$$
\begin{aligned}
F & =\zeta_{\tau} \widetilde{\mathscr{L}}(x, \mathscr{D}) v+\left[\widetilde{\mathscr{L}}, \zeta_{\tau}\right] v+\left(\mathscr{L}_{0}(\mathscr{D})-\widetilde{\mathscr{L}}(x, \mathscr{D})\right) \zeta_{\tau} v \\
\Phi & =\zeta_{\tau} \widetilde{\mathscr{B}}(x, \mathscr{D}) v+\left[\widetilde{\mathscr{B}}, \zeta_{\tau}\right] v+\left(\mathscr{B}_{0}(\mathscr{D})-\widetilde{\mathscr{B}}(x, \mathscr{D})\right) \zeta_{\tau} v
\end{aligned}
$$

Здесь $\left[\widetilde{\mathscr{L}}, \zeta_{\tau}\right],\left[\widetilde{\mathscr{B}}, \zeta_{\tau}\right]-$ коммутаторы, $\widetilde{\mathscr{L}}$ и $\widetilde{\mathscr{B}}$ имеют тот же смысл, что $\widetilde{\mathscr{L}}_{0}$ и $\widetilde{\mathscr{B}}_{0}$ в (6.6). (Мы учли, что $x^{\tau}(\xi)=\mathbf{1}$ при $\xi=\tau$.) Отличие (6.12) от соответствуюшего уравнения из $[10$, с. 109$]$ объясняется тем, что у нас коэффициенты оператора $\mathscr{L}$ принадлежат $C^{\infty}(\bar{Q})$.

Применяя те же преобразования, что и в $[10$, с. 109-110], к задаче $(6.12),(6.13)$ в угле $K^{\tau}$, получим

$$
\begin{aligned}
c_{2}\left\|\zeta_{\tau} v ; X_{\beta}^{n}(Q)\right\| \leqslant & \left\|\zeta_{\tau} \mathscr{L} v ; X_{\beta-a}^{n}(Q)\right\| \\
& +\left\|\zeta_{\tau} \mathscr{B} v ; Y_{\beta-b}^{n}(\partial Q \backslash \mathscr{J})\right\|+c(\tau, \delta)\left\|v ; X_{\beta}^{n-1}(Q)\right\|,
\end{aligned}
$$

где $c(\tau, \delta)>0$ - константа, которую можно сделать сколь угодно малой за счет выбора $\delta$-окрестности точки $\tau$, где сосредоточена $\zeta_{\tau}$. Из (6.11) и (6.14), очевидно, имеем (6.10), что и требовалось доказать.

Используя оценку (6.10), аналогично $§ 3$ (при этом для доказательства аналога теоремы 3.2 нужно использовать лемму 6.1) получим, что имеет место 
Теорема 6.1. Для нётеровости задачи Римана-Гильберта (6.3) необходимо и достаточно выполнения условий 1)-6) и условия: на прямой $\operatorname{Re}(i \lambda)=\beta_{\tau}$ нет точек спектра пучка $(6.9) \mathscr{U}^{\tau}(\lambda), \tau \in \mathscr{J}$. При выполнении этих условий индекс задачи не зависит от $n, p, \mu$ ( $n>\sigma_{0}$ или $n \geqslant \max \left\{0, \sigma_{0}\right\}, 1<p<+\infty$, $0<\mu<1$ ), а также от пространств, над которыми рассматривается задача (они могут быть как весовыми пространствами Соболева, так и Гёльдера).

Отметим еше раз, что теорема доказывается аналогично теореме 3.4. Доказательство нётеровости задачи для пространств Соболева в случае $p=2$ можно найти в $[10$, с. 108-114]. Разница между формулировками теоремы из [10] и теоремы 6.1 связана с определением пространств. Пространства $V_{\gamma}^{n}$ из [10] и пространства $W_{2, \beta}^{n}$ связаны равенством $\gamma=n-\beta-1$.

\section{§7. Задача Пуанкаре}

\section{1. Краевая задача Римана-Гильберта для одной правильно эллип-} тической системы. Рассмотрим задачу

$$
\left\{\begin{array}{l}
\frac{\partial u_{1}}{\partial x_{1}}-\frac{\partial u_{2}}{\partial x_{2}}=0 \\
\cdots \cdots \cdots \cdots \cdots \cdots \\
\frac{\partial u_{r}-1}{\partial x_{1}}-\frac{\partial u_{r}}{\partial x_{2}}=0 \\
\frac{\partial u_{r}}{\partial x_{1}}+\mathbf{a}_{1} \frac{\partial u_{1}}{\partial x_{2}}+\cdots+\mathbf{a}_{r} \frac{\partial u_{r}}{\partial x_{2}}=f_{r} \in X_{(\gamma-1)}^{n}(Q), \\
\quad G u=\varphi \in Y_{(\gamma)}^{n}(\partial Q \backslash \mathscr{J}), \quad u \in X_{(\gamma)}^{n+1}(Q),
\end{array}\right.
$$

где $\mathbf{a}_{j}(j=1, \ldots, r)$ - квадратные матрицы порядка $\ell$ и $G$ - прямоугольная $(k \times 2 k)$-матрица, $\ell r=2 k, f_{r}, u_{j}(j=1, \ldots, r)-k$-компонентные вектор-функции, $u=\left(u_{1}, \ldots, u_{r}\right)$ - искомая, $\varphi-k$-вектор-функция. Условие

$$
\operatorname{det}\left(\mathbf{1} \xi_{1}^{r}+\sum_{j=1}^{r} \mathbf{a}_{j}(x) \xi_{1}^{j-1} \xi_{2}^{r+1-j}\right) \neq 0, \quad x \in \bar{Q}, \quad \xi \in \mathbb{R}^{2} \backslash\{0\}
$$

есть условие эллиптичности системы (7.1), которое считаем выполненным. Представим систему (7.1) в виде (5.12). И пусть для задачи (7.1), (7.2) выполнены условия из п. $2 \S 5$. Пространства, над которыми рассматривается задача $(7.1),(7.2),-$ пространства $\ell$ - и $\ell r$-вектор-функций соответственно. Они определены в п. $1 \S 5$.

Пусть выполнено условие (4.15); тогда имеет место

ЛЕмма 7.1. Для нётеровости задачи (7.1), (7.2) необходимо и достаточно выполнения условий из теоремы 5.2. При выполнении последних индекс задачи дается формулой (5.14), әде $2 k=\ell r, \beta=\gamma$. 
ДоказАТЕЛЬСтво. Пусть $\mathscr{L}$ - оператор задачи (7.1), (7.2), $\mathscr{A}$ - оператор задачи $(7.1),(7.2)$, где в правых частях $(7.1)$ вместо нулей имеем $f_{1}, \ldots, f_{r-1} \in$ $X_{(\gamma-1)}^{n}(Q)$, и пусть $\mathscr{A}_{1}$ - оператор задачи $(7.1),(7.2)$ с $f_{r}=0$. Очевидно, что $\operatorname{Ker} \mathscr{A}=\operatorname{Ker} \mathscr{A}_{1}=\operatorname{Ker} \mathscr{L}$. Так как пространства $\Re_{1}, \Re_{2}, \Re_{3}$ правых частей рассматриваемых задач $\left(\Re_{1}\right.$ соответствует $\left.\mathscr{A}_{1}, \Re_{2}-\mathscr{L}, \Re_{3}-\mathscr{A}\right)$ связаны включениями $\Re_{1} \subset \Re_{2} \subset \Re_{3}$, для сопряженных пространств имеем обратные включения $\Re_{3}^{*} \subset \Re_{2}^{*} \subset \Re_{1}^{*}$. Следовательно, $\operatorname{Ker} \mathscr{A}^{*} \subset \operatorname{Ker} \mathscr{L}^{*} \subset \operatorname{Ker} \mathscr{A}_{1}^{*}$. Отсюда с учетом предыдуших соотношений для ядер $\mathscr{A}, \mathscr{A}_{1}, \mathscr{L}$ и замечания 5.1 получим требуемое. Лемма доказана.

2. Об одном представлении функций. Пусть $Q-(m+1)$-связная область плоскости с кусочно гладкой гранищей, состоящей из замкнутых контуров $L_{0}, \ldots, L_{m}$, причем $L_{0}$ содержит внутри себя остальные. И пусть $U_{i}^{1}, i=1, \ldots, r$, $r \geqslant 2,-N$-вектор-функции, принадлежашие $H_{\mu,(\beta)}^{n}(Q), 0<\mu<1, n \geqslant 1-$ натуральное число. Причем в $\bar{Q} \backslash \mathscr{J}$ имеем

$$
\frac{\partial U_{i}^{1}}{\partial x_{1}}=\frac{\partial U_{i+1}^{1}}{\partial x_{2}}, \quad i=1, \ldots, r-1 .
$$

Рассмотрим в $\bar{Q} \backslash \mathscr{J}$ переопределенную систему

$$
\mathscr{D}_{2}^{r-i} \mathscr{D}_{1}^{i-1} v=U_{i}^{1}, \quad i=1, \ldots, r .
$$

Требуется определить (однозначные) решения этой системы. Справедлива

ЛЕмма 7.2. Для разрешимости в $H_{\mu,(\beta+r-1)}^{n+r-1}(Q)$ системы (7.4), необходимо и достаточно выполнения:

a) условий (7.3);

b) того, что весовой порядок такой, что $\beta_{\tau} \neq 1-\mathbf{r}, \quad \tau \in \mathscr{J}$;

c) $k m r(r-1) / 2$ условий

$$
\int_{\tilde{L}_{j}} U_{i+1}^{s} d x_{1}+U_{i}^{s} d x_{2}=0, \quad U_{i}^{s+1}=C_{i}^{s}+\int_{x_{0}}^{x} U_{i+1}^{s} d x_{1}+U_{i}^{s} d x_{2},
$$

$j=1, \ldots, m, i=1, \ldots, r-s, s=1, \ldots, r-1$, әде $\tilde{L}_{j} \subset \bar{Q}, j=1, \ldots, m,-$ любая фиксированная кривая, совпадающая с $L_{j}$, за исключением малой окрестности $\mathscr{J}, C_{i}^{s}-$ вектор с произвольныцми числовыми әлементами из $\mathbb{C}$.

При выполнении этих условий решение дается формулой

$$
v=P(x)+K\left(U_{1}^{1}, \ldots, U_{r}^{1} ; x\right), \quad x \in \bar{Q} \backslash \mathscr{J}, \quad v \in H_{\mu,(\beta)}^{k}(Q),
$$

$k=n+r-1$, где $P(x)$ - вектор, компоненты которого есть полиномы переменных $x_{1}, x_{2}$ с произвольными комплексными коэффициентами, $K$ определяется рекурентной формулой

$$
\begin{aligned}
& V_{i}^{s+1} \stackrel{\text { def }}{=} \int_{x_{0}}^{x} V_{i+1}^{s} d x_{1}+V_{i}^{s} d x_{2}, \\
& V_{i}^{1}=U_{i}^{1}, \quad i=1, \ldots, r-s, \quad s=1, \ldots, r-1, \\
& K=V_{1}^{r}=\int_{x_{0}}^{x} V_{2}^{r-1} d x_{1}+V_{1}^{r-1} d x_{2} .
\end{aligned}
$$


(Здесь криволинейные интегралы берутся по (любой) кусочно гладкой кривой, соединяющей точки $x_{0}, x \in \bar{Q} \backslash \mathscr{J}$, причем $x_{0}$ - произвольная фиксированная точка $Q$.)

ДокАЗАТЕЛЬСтво. Очевидно, что $v$ принадлежит $H_{\mu}^{k}$ вне любой окрестности $\mathscr{J}$ (см. также [12]). Поэтому достаточно доказать, что $v=P_{\tau}+v_{\tau}, v_{\tau} \in$ $H_{\mu, \beta_{\tau}}^{k}\left(Q_{\tau}\right)$, где $P_{\tau}$ - многочлен от $x_{1}, x_{2}$ степени $k(\tau)<\beta_{\tau}$ (см. $\S 1$, определение $\left.H_{\mu,(\beta)}^{k}\right)$. В силу индуктивного способа определения пространства $H_{\mu,(\beta)}^{k}$ и ввиду рекуррентности формул (7.6), (7.7) достаточно ограничиться случаем $r=2$, $n=1$. Более того, так как сейчас нас интересует поведение функции $v$, заданной формулой (7.6), вблизи $\tau$, можно считать $n=0$. Тогда (7.6) переходит в равенство

$$
v=c+\int_{x_{0}}^{x} U_{2}^{1} d x_{1}+U_{1}^{1} d x_{2}
$$

Пусть $\beta_{\tau}<-1$. Так как $\mathscr{D}_{1} v=U_{2}^{1}, \mathscr{D}_{2} v=U_{1}^{1}$ принадлежат $H_{\mu,\left(\beta_{\tau}\right)}\left(Q_{\tau}\right)$, нужно доказать, что $v \in H_{\mu,\left(\beta_{\tau}+1\right)}\left(Q_{\tau}\right)$. Из (7.6) следует, что $v$ представляется в виде

$$
v(x)=P_{\tau}+v_{\tau}, \quad v_{\tau}=\int_{x}^{\tau} U_{2}^{1} d x_{1}+U_{1}^{1} d x_{2},
$$

где $P_{\tau}$ - константа. Отсюда с учетом оценок $\left|U_{i}^{1}\right| \leqslant C_{i} r^{\beta_{\tau}}, i=1,2, r=|x-\tau|$, получим требуемое.

Пусть теперь $\beta_{\tau}>-1$. В данном случае константы принадлежат пространству $H_{\mu,\left(\beta_{\tau}+1\right)}$. С учетом этого и оценок подынтегральных функций из (7.8) получим, что интеграл принадлежит $H_{\mu,\left(\beta_{\tau}+1\right)}\left(Q_{\tau}\right)$ - требуемый результат.

(Случай $\beta_{\tau}=-1$ исключительный. В данном случае при интегрировании могут появиться логарифмические особенности.) Лемма доказана.

3. Задача Пуанкаре для правильно эллиптических систем. В ограниченной области $Q$ плоскости (описание $Q$ см. $\S 3$, п. 1 ) рассмотрим следующую задачу для однородной эллиптической системы порядка $r$ :

$$
\begin{aligned}
& A v \equiv \sum_{j=1}^{r+1} \mathbf{a}_{j}\left(\frac{\partial}{\partial x_{2}}\right)^{r-j+1}\left(\frac{\partial}{\partial x_{1}}\right)^{j-1} v=f \in X_{(\beta-r)}^{n}(Q), \\
& B v \equiv \sum_{j=1}^{r} \mathbf{b}_{j}\left(\frac{\partial}{\partial x_{2}}\right)^{r-j}\left(\frac{\partial}{\partial x_{1}}\right)^{j-1} v=\varphi \in Y_{(\beta)}^{n}(\partial Q \backslash \mathscr{J}),
\end{aligned}
$$

$v \in X_{(\beta)}^{n+r}(Q)$, где $\mathbf{a}_{1}, \ldots, \mathbf{a}_{r+1}-$ квадратные матрицы порядка $\ell$, причем

$$
L(x, \xi)=\operatorname{det}\left(\sum_{j=1}^{r+1} \mathbf{a}_{j}(x) \xi_{1}^{j-1} \xi_{2}^{r+1-j}\right) \neq 0, \quad x \in \bar{Q}, \quad \xi \in \mathbb{R}^{2} \backslash\{0\} .
$$

В силу условия эллиптичности (7.11) матрица $\mathbf{a}_{r+1}$ обратима, поэтому без ограничения общности полагаем $\mathbf{a}_{r+1}=\mathbf{1}$. Предполагаем также, что (7.9) - правильно эллиптическая система, т.е. корни многочлена $P(\lambda)=\operatorname{det}\left(\Sigma \mathbf{a}_{j}(-\lambda)^{j}\right)$ 
(с учетом кратностей) расположены поровну в верхней и нижней полуплоскостях. Значит, число корней $\ell r=2 N$ - четное. Матрицы $\mathbf{b}_{1}, \ldots, \mathbf{b}_{r}$ - прямоугольные $(N \times \ell)$-матрищы, принадлежашие $C^{\infty}(\partial Q \backslash \mathscr{J})$, причем они имеют левые и правые продолжения (каждое класса $C^{\infty}$ ) в точки $\mathscr{J}$.

Пространства, над которыми рассматривается задача, - это весовые пространства $\ell$-вектор-функций Соболева или Гёльдера. Они получены заменой пространств в правых частях (6.4), (6.5) (где $t_{1}=\cdots=t_{\ell}=r, s_{1}=\cdots=s_{\ell}=0$, $\left.\sigma_{1}=\cdots=\sigma_{k}=-1\right)$ на соответствуюшие конечномерные расширения $\left(W_{p, \beta}^{k}\right.$ заменяем на $W_{p,(\beta)}^{n}$, а $H_{\mu, \beta}^{n}$ на $\left.H_{\mu,(\beta)}^{n}\right)$. Ввиду последнего к задаче $(7.9),(7.10)$ можно применить результаты $\S 6$. В частности, задача (7.9), (7.10) тогда и только тогда нётеровая, когда выполнены условия 1)-6) 66 и условие о спектре пучка из теоремы 6.1 .

Вместе с задачей (7.9), (7.10) рассмотрим задачу (7.1), (7.2), где $\gamma=\beta-r+1$, $\mathbf{a}_{j}, j=1, \ldots, r,-$ такие же, как в $(7.9)$, a $G_{0}=\left(\mathbf{b}_{1}, \ldots, \mathbf{b}_{r}\right)$. В дальнейшем эту задачу называем задачей $A$.

Пусть $\mathscr{X}_{j}(\zeta)=\operatorname{diag}\left(\mathscr{X}_{1 j}(\zeta), \mathscr{X}_{2 j}(\zeta)\right), \tau_{i}=\tau \in \mathscr{J},-$ конщевой символ задачи A. Он определяется по формулам (5.13), с учетом того, что в (5.11) $\mathbf{a}=\mathbf{1}$, a $\mathbf{b}$ определяется равенством

$$
\mathbf{b}=\left(\begin{array}{ccccc}
\mathbf{0} & -\mathbf{1} & \mathbf{0} & \ldots & \mathbf{0} \\
\mathbf{0} & \mathbf{0} & -\mathbf{1} & \ldots & \mathbf{0} \\
\ldots & \ldots & \ldots \ldots & \ldots & \ldots \\
\mathbf{0} & \mathbf{0} & \mathbf{0} & \ldots & -\mathbf{1} \\
\mathbf{a}_{1} & \mathbf{a}_{2} & \mathbf{a}_{3} & \ldots & \mathbf{a}_{r}
\end{array}\right)
$$

где $\mathbf{0}$ и $\mathbf{1}$ - нулевая и единичная матрицы порядка $\ell$. (Из (7.11) следует эллиптичность матрищы $\mathbf{b}$. Поэтому для нее в $\bar{Q}$ имеет место разложение $(2.1): \mathbf{b}=$ $T \operatorname{diag}\left(\Lambda_{1}, \Lambda_{2}\right) T^{-1}, T=\left(T_{1}, T_{2}\right)$.) Пусть

$$
\operatorname{det} \aleph_{1} \operatorname{det} \aleph_{2} \neq 0 \quad \text { всюду на } \quad \partial Q \backslash \mathscr{J},
$$

где $\aleph_{1}=\bar{G} \bar{T}_{1}, \aleph_{2}=G T_{2}-$ квадратные матрищы порядка $N$. И пусть

$$
\operatorname{det} \aleph_{1}(\tau \pm 0) \operatorname{det} \aleph_{2}(\tau \pm 0) \neq 0, \quad \tau \in \mathscr{J}
$$

Всюду в дальнейшем предполагаем выполненным условие

$$
\beta_{\tau}-r+1 \notin \mathbb{N} \cup\{0\}, \quad \tau \in \mathscr{J},
$$

где $\mathbb{N}$ - множество натуральных чисел.

Справедлива 
Теорема 7.1. Задача Пуанкаре (7.9), (7.10) тогда и только тогда нётеровая, когда выполнены условия (7.13), (7.14) и условие: на прямой $\operatorname{Re} \zeta=$ $\beta_{\tau}-r+1$ определитель $\operatorname{det} \mathscr{X}_{j}(\zeta)$ не равен нулю, $\tau=\tau_{j} \in \mathscr{J}$. При выполнении этих условий индекс задачи дается формулой

$$
\text { ind } \mathscr{A}=2^{-1}\left(\sum_{l=1}^{2}\left(\operatorname{Ind}_{\Gamma} \bar{\aleph}_{l}^{-1} \aleph_{l}-\sum_{j=1}^{|\mathscr{J}|} \operatorname{Ind}_{\beta_{j}-r+1} \mathscr{X}_{l j}(\zeta) y_{l j}^{-1}(\zeta)\right)-\ell r^{2}(m-1)\right) \text {, }
$$

әде $m+1$ - число связных компонент границы, $\ell$ - число уравнений системы (7.9), $r$ - порядок системы, $|\mathscr{J}|$ - число точек $\mathscr{J}, \beta_{j}=\beta_{\tau_{j}}, \tau_{j}=\tau \in \mathscr{J}$.

Пусть $\beta=\left\{\beta_{\tau}\right\}$ и $\gamma=\left\{\gamma_{\tau}\right\}$ - допустимые весовые порядки, т.е. на прямых $\operatorname{Re} \zeta=\beta_{\tau}, \quad \operatorname{Re} \zeta=\gamma_{\tau}, \quad \tau \in \mathscr{J}$, нет нулей $\operatorname{det} \mathscr{X}_{j}(\zeta)$, и пусть выполнены условия (7.13), (7.14). Тогда индексы задачи (7.9), (7.10) связаны равенством

$$
\text { ind } \mathscr{A}_{\gamma}-\text { ind } \mathscr{A}_{\beta}=\sum_{\tau \in \mathscr{J}} S_{\tau}
$$

где $\mathscr{A}_{\gamma}$ - оператор задачи (7.9), (7.10) при $\beta=\gamma, \pm S_{\tau}$ равен числу нулей концевого символа $\mathscr{X}_{j}(\zeta), \tau_{j}=\tau \in \mathscr{J}$ (с учетом кратности) между прямыими $\operatorname{Re} \zeta=\beta_{\tau}-r+1, \operatorname{Re} \zeta=\gamma_{\tau}-r+1$. Знак "-" соответствует случаю $\beta_{\tau} \leqslant \gamma_{\tau}$, а знак "+" - случаю $\beta_{\tau} \geqslant \gamma_{\tau}$.

ДоКАЗАТЕЛЬСТВо. Ввиду теоремы 6.1 без ограничения обшности можно считать, что пространства, над которыми рассматривается задача, есть весовые пространства Гёльдера. (Пространства из (7.9), (7.10), как было отмечено, есть конечномерные расширения соответствуюших пространств из $\S 6$, а при таких расширениях свойство нётеровости сохраняется.)

Покажем, что задача Пуанкаре эквивалентна в смысле нётеровости следующей задаче $B$.

Задача $B$ такая же, как задача $A$, но теперь искомая $u=\left(u_{1}, \ldots, u_{r}\right)$ принадлежит подпространству $V$ пространства $H_{\mu,(\gamma)}^{n+1}(Q), \gamma=\beta-r+1$, определенному равенством

$$
V=\left\{u \in H_{\mu,(\gamma)}^{n+1}(Q) \mid \text { для } U_{i}^{1} \text { выполнены условия }(7.5)\right\},
$$

где $U_{i}^{1}=u_{i}, i=1, \ldots, r$.

Пусть $u \in V$-решение задачи $B$. Тогда ввиду (7.1) вектор $U=u$ удовлетворяет условиям (7.3). Так как $u \in V$, выполнены и условия (7.5). Значит, по лемме 7.2 найдется вектор $v \in H_{\mu,(\beta)}^{n+r}(Q)$, для которого имеем

$$
u_{i}=\left(\frac{\partial}{\partial x_{2}}\right)^{r-i}\left(\frac{\partial}{\partial x_{1}}\right)^{i-1} v, \quad i=1, \ldots, r
$$

Внеся $u_{i}$ в задачу $B$, получим, что $v$ есть решение задачи (7.9), (7.10). Обратно, пусть теперь $v$ - решение задачи $(7.9),(7.10)$. Тогда вектор $u=\left(u_{1}, \ldots, u_{r}\right)$, 
определенный формулой (7.18), есть решение задачи $B$ ввиду леммы 7.2. Таким образом, задача $B$ и задача Пуанкаре разрешимы или неразрешимы одновременно, и в случае нормальной разрешимости дефектные числа соответствуюших им операторов совпадают.

Как и в [12], для случая гладких областей и обычных пространств Гёльдера, легко получим, что из конечномерности пространства решений однородной задачи $B$ следует то же для задачи Пуанкаре, и обратно. И в случае конечномерности размерность второго на $\ell r(r-1) / 2$ больше первого.

Итак, задача Пуанкаре и задача $B$ нётеровые или не нётеровые одновременно, и в случае нётеровости индексы их связаны равенством

$$
\text { ind } \mathscr{A}=\text { ind } \mathscr{L}_{1}+\frac{\ell r(r-1)}{2}
$$

где $\mathscr{L}_{1}$ - оператор, соответствующий краевой задаче $B$. Заметим теперь, что $H_{\mu,(\gamma)}^{n+1}(Q)$ является конечномерным расширением $V$, а именно $(\ell m r(r-1) / 2)$-мерным. Следовательно, ind $\mathscr{L}_{1}=$ ind $\mathscr{L}-\ell m r(r-1) / 2$, где $\mathscr{L}$ - оператор задачи $A$. Отсюда согласно лемме 7.1 получим требуемое. Аналогично получим справедливость и второй части теоремы. Теорема доказана.

4. $\mathbb{R}$-линейные задачи. Рассмотрим сначала задачу Пуанкаре (7.9), (7.10), где коэффициенты уравнений и граничных условий - действительные функции. Тогда из условия эллиптичности следует правильная эллиптичность. Согласно $(2.1)$ в разложении эллиптической матрицы $\mathbf{b}=T \operatorname{diag}\left(\Lambda_{1}, \Lambda_{2}\right) T^{-1}, \quad T=$ $\left(T_{1}, T_{2}\right)$, можно считать $T_{2}=\bar{T}_{1}, \Lambda_{1}=\Lambda, \Lambda_{2}=\bar{\Lambda}$. В силу этого матрицы $\aleph_{1}$ и $\aleph_{2}$ равны. Следовательно, условия $(7.13),(7.14)$ здесь эквивалентны условиям

$$
\begin{gathered}
\operatorname{det} \aleph \neq 0 \quad \text { всюду на } \quad \partial Q \backslash \mathscr{J}, \\
\operatorname{det} \aleph(\tau \pm 0) \neq 0, \quad \tau \in \mathscr{J},
\end{gathered}
$$

где $=G \bar{T}_{1}$. Для конщевого символа $\widetilde{\mathscr{X}}_{j}(\zeta)=\operatorname{diag}\left(\mathscr{X}_{1 j}(\zeta), \mathscr{X}_{2 j}\right)$ согласно $(6.13)$ имеем

$$
\mathscr{X}_{2 j}(\zeta)=\mathscr{X}_{1 j}(\zeta) \stackrel{\text { def }}{=} \mathscr{X}_{j}(\zeta) .
$$

(Здесь мы учли, что для задачи с действительньми коэффициентами справедливы равенства $\Lambda_{2}=\bar{\Lambda}_{1}, T_{2}=\bar{T}_{1}, \aleph_{2}=\aleph_{1}$.) Отсюда и из теоремы 7.1 следует справедливость следуюшей теоремы.

Теорема 7.2. Для нётеровости задачи (7.9), (7.10) с действительными коэффициентами необходимо и достаточно выполнения (7.19), (7.20) и условия: на прямой $\operatorname{Re} \zeta=\beta_{\tau}-r+1$ нет корней определителя концевого символа $\mathscr{X}_{j}(\zeta), \tau=\tau_{j} \in \mathscr{J}$. При выполнении әтих условий индекс задачи дается равенством

$$
\text { ind } \mathscr{A}=\operatorname{Ind}_{\Gamma} \bar{\aleph}^{-1} \aleph-\sum_{j=1}^{|\mathscr{F}|} \operatorname{Ind}_{\beta_{j}-r+1} \widetilde{\mathscr{X}}_{j}(\zeta) y_{j}^{-1}-\frac{\ell r^{2}(m-1)}{2} \text {. }
$$


(Теорема дополняется и второй частью, аналогичной теореме 7.1.)

Заметим, что матрицы $y_{1 j}(\zeta), y_{2 j}(\zeta)$ в данном случае можно выбрать одинаковыми.

Рассмотрим теперь $\mathbb{R}$-линейную задачу:

$$
\begin{gathered}
A v \equiv \sum_{j=1}^{r+1} \mathbf{a}_{j}\left(\frac{\partial}{\partial x_{2}}\right)^{r-j+1}\left(\frac{\partial}{\partial x_{1}}\right)^{j-1} v=f \in X_{(\beta-r)}^{n}(Q), \\
B v \equiv \operatorname{Re}\left(\sum_{j=1}^{r} \mathbf{b}_{j}\left(\frac{\partial}{\partial x_{2}}\right)^{r-j}\left(\frac{\partial}{\partial x_{1}}\right)^{j-1} v\right)=\varphi \in Y_{(\beta)}^{n}(\partial Q \backslash \mathscr{J}), \\
v \in X_{(\beta)}^{n+r}(Q),
\end{gathered}
$$

где $\mathbf{a}_{1}, \ldots, \mathbf{a}_{r+1}=\mathbf{1}$ - квадратные матрицы порядка $\ell$, принадлежащие $C^{\infty}(\bar{Q})$, причем для них выполнено условие эллиптичности (7.11). (Правильная эллиптичность не требуется.)

Матрицы $\mathbf{b}_{1}, \ldots, \mathbf{b}_{r}$ такие же, как в п. 2 , но теперь они прямоугольные $(\ell r \times r)$-матрицы. Определим матрицу $\mathbf{b}$ формулой (7.12). Она допускает разложение (2.1): $\mathbf{b}=T \operatorname{diag}\left(\Lambda_{1}, \Lambda_{2}\right) T^{-1}, T=\left(T_{1}, T_{2}\right)$. Пусть

$$
\begin{gathered}
\operatorname{det} \aleph \neq 0 \quad \text { всюду на } \quad \partial Q \backslash \mathscr{J}, \\
\operatorname{det} \aleph(\tau \pm 0) \neq 0, \quad \tau \in \mathscr{J},
\end{gathered}
$$

где $\aleph=\left(\bar{G}_{1}, G T_{2}\right), G=\left(\mathbf{b}_{1}, \ldots, \mathbf{b}_{r}\right)$. Задаче $(7.22),(7.23)$ поставим в соответствие задачу Римана-Гильберта для эллиптической системы $k=\ell r$ уравнений

$$
\mathscr{D}_{1} u+\mathbf{b} \mathscr{D}_{2} u=g, \quad \operatorname{Re}(G u)=\psi,
$$

где $\mathbf{b}$ определена формулой (7.12). Пусть $\mathscr{X}_{j}(\zeta), \tau_{j}=\tau \in \mathscr{J},-$ конщевой символ этой задачи (он определен в п. $1 \S 5$, см. $\left.(5.6)-(5.8),\left(5.6^{\prime}\right),\left(5.6^{\prime \prime}\right)\right)$. Тогда имеет место

Tеорема 7.3. Задача (7.22), (7.23) тогда и только тогда нётеровая, когда выполнены (7.24), (7.25) и на прямой $\operatorname{Re} \zeta=\beta_{\tau}-r+1$ нет корней определителя $\operatorname{det} \mathscr{X}_{j}(\zeta), \quad \tau=\tau_{j} \in \mathscr{J}$. При выполнении этих условий индекс задачи дается равенством

$$
\operatorname{ind} \mathscr{A}=\operatorname{Ind}_{\Gamma} \bar{\aleph}^{-1} \aleph-\sum_{j=1}^{|\mathscr{J}|} \operatorname{Ind}_{\beta_{j}-r+1} \mathscr{X}_{j}(\zeta) y_{j}^{-1}-\ell r^{2}(m-1)
$$

Теорема доказывается так же, как теорема 5.1, с привлечением теоремы 7.2, поэтому подробности опускаем. Ее также можно дополнить и второй частью, аналогичной теореме 7.1 . 
СлЕДСТВИЕ 7.1. Пусть все корни характеристического полинома $P(\lambda)=$ $\Sigma \mathbf{a}_{j}(-\lambda)^{j}$ расположены или в верхней полуплоскости, или в нижней. Тогда в первом случае в формуле $(7.26) \aleph=\bar{G}$, а концевой символ определяется равенством (5.6"), где $\mathbf{a}=\mathbf{1}, \mathbf{b}$ из (7.12). А во втором случае $\aleph=G, a$ концевой символ определяется равенством (5.6.

Доказательство такое же, как доказательство следствия 5.1. Заметим, что формулы (7.21), (7.26) есть следствия формулы (7.16). Применение последней к конкретным задачам (удовлетворяющим условиям из данного пункта) сложнее, чем к (7.21), (7.26), так как приходится оперировать матрицами в два раза больших размеров, чем в (7.21), (7.26). Заметим также, что в данном параграфе (и ниже) рассматриваются задачи без младших членов. При естественных условиях на коэффициенты они образуют компактную добавку к оператору задачи (7.9), (7.10) и др. Следовательно, последнее обстоятельство не влияет на наши результаты.

\section{§8. Краевые задачи $\mathbb{k}-г о$ порядка}

В первых двух пунктах рассматриваются задачи, порядки краевых условий которых $\mathbb{k}$ меньше порядка системы $r$, а в третьем пункте $\mathbb{k} \geqslant r$. Всюду в параграфе считаем, что выполнено условие (7.15), где $\beta_{\tau}-r+1$ заменено на $\beta_{\tau}-\mathbb{k}$.

1. Для эллиптической системы (7.9) рассмотрим краевую задачу

$$
\begin{gathered}
A v=f \in X_{(\beta-r)}^{n}(Q), \quad v \in X_{(\beta)}^{n+r}(Q), \\
B v \equiv \sum_{j=1}^{\mathbb{k}+1} \mathbf{b}_{j}^{0}\left(\frac{\partial}{\partial x_{2}}\right)^{r-j}\left(\frac{\partial}{\partial x_{1}}\right)^{j-1} v=\varphi \in Y_{(\beta-\mathrm{k})}^{n-\mathrm{k}}(\partial Q \backslash \mathscr{J}) .
\end{gathered}
$$

Коэффициенты системы и краевых условий - действительные функции. Все предположения п. $4 \S 7$ (где $\mathbf{b}_{j}=\mathbf{b}_{j}^{0}$ ) сохраняем.

Пусть $G_{0}$ - прямоугольная $(N \times 2 N)$-матрица $(2 N=\ell r)$, определенная формулой $G_{0}=\left(\mathbf{b}_{1}^{0}, \ldots, \mathbf{b}_{\mathbb{k}+1}^{0}, \mathbf{0}, \ldots, \mathbf{0}\right)$, где $\mathbf{0}$ - нулевая $(N \times 2 N)$-матрища, причем нулевые блоки заполняют последние $r-\mathbb{k}-1$ мест. Матрица $T=\left(T_{1}, \bar{T}_{1}\right)$ и концевой символ $\mathscr{X}_{j}^{0}(\zeta)$ такие же, как в п. $4 \S 7$ (с заменой $\mathbf{b}_{j}$ на $\mathbf{b}_{j}^{0}$ ). Имеет место

ТЕОРема 8.1. Задача (8.1), (8.2) тогда и только тогда нётеровая, когда выполнены условия (7.19), (7.20) (с заменой $\mathbf{b}_{j}$ на $\mathbf{b}_{j}^{0}$ ) и на прямой $\operatorname{Re} \zeta=$ $\beta_{j}-\mathbb{k}, \quad \beta_{j}=\beta_{\tau_{j}}$, нет корней определителя концевого символа $\mathscr{X}_{j}^{0}(\zeta), \tau=$ $\tau_{j} \in \mathscr{J}$. При выполнении этих условий индекс задачи дается формулой

$$
\text { ind } \mathscr{A}=\operatorname{Ind}_{\Gamma} \bar{\aleph}_{0}^{-1} \aleph_{0}-\sum_{j=1}^{s} \operatorname{Ind}_{\beta_{j}-\mathbb{k}}\left(\mathscr{X}_{j}^{0} y_{j}^{-1}\right)-\frac{r \ell(2 \mathbb{k}-r+2)(m-1)}{2} \text {. }
$$

(Здесь $\aleph_{0}=G_{0} \bar{T}_{1}$. Другие обозначения те же, что и в теореме 7.1. Как это, так и другие утверждения параграфа дополняются и второй частью, аналогичной теореме 7.1. Они получаются из нее заменой $r-1$ на $\mathbb{k}$.) 
ДокАЗАТЕЛЬСТво. Так как случай $\mathbb{k}=r-1$ рассматривался в $\S 7$, считаем, что $\mathbb{k} \leqslant r-2$. Согласно теореме 6.1 достаточно ограничиться случаем $H_{\mu,(\gamma)}^{n}$-пространств. Как и в [12], изучим задачу (8.1), (8.2) сведением к задаче Пуанкаре (7.9), (7.10). Подействуем на граничные условия $p=r-\mathbb{k}-1$ раз операцией дифференцирования по дуге $\partial / \partial \sigma$ при естественной параметризации. Соответствующий такому преобразованию оператор, в отличие от [12], имеет ненулевой индекс $s p N$, где $s=|\mathscr{J}|$-число точек $\mathscr{J}$. В результате получим задачу Пуанкаре, эквивалентную исходной в смысле нётеровости. Обозначим $\mathscr{A}_{0}$ оператор, соответствующий преобразованной задаче. В случае нётеровости индексы операторов $\mathscr{A}$ и $\mathscr{A}_{0}$ связаны равенством

$$
\text { ind } \mathscr{A}_{0}=\text { ind } \mathscr{A}+p N|\mathscr{J}|, \quad p=r-\mathbb{k}-1 .
$$

Для нётеровости $\mathscr{A}_{0}$ необходимо и достаточно выполнения условий, отмеченных в теореме 7.2.

Покажем, что:

1) они эквивалентны условиям теоремы 8.1 ;

2) формула (7.21), с учетом (8.4), преобразуется в (8.3).

Легко видеть, что на $\partial Q \backslash \mathscr{J}$ имеем (см. [12])

$$
\aleph=G \bar{T}_{1}=\left(G_{0} \bar{T}_{1}\right)\left(\sigma_{2} \mathbf{1}-\sigma_{1} \bar{\Lambda}\right)^{p},
$$

где $\left(\sigma_{1}, \sigma_{2}\right)$ - единичный вектор касательной, направленный в сторону возрастания дуг, 1 - единичная матрица порядка $N$. Отсюда следует справедливость 1 ), за исключением условия на конщевой символ. Изучим последний. Аналогично (8.5) имеем

$$
\begin{gathered}
C^{-1}\left(\tau_{j}-0\right)=\bar{D}_{k}^{-p} C_{0}^{-1}\left(\tau_{j}-0\right) D_{k}^{p}, \quad C\left(\tau_{j}+0\right)=D_{r}^{-p} C_{0}\left(\tau_{j}+0\right) \bar{D}_{r}^{p}, \\
D_{i}=\operatorname{Im} q_{i}-\Lambda \operatorname{Re} q_{i}, \quad i=k, r
\end{gathered}
$$

где $C_{0}=\bar{A}^{-1} A, A=G_{0} \overline{\mathscr{T}}_{1}$. (По поводу остальных обозначений см. п. $1 \S 4$.) Следовательно, конщевой символ задачи Пуанкаре представляется в виде

$$
\mathscr{X}_{j}(\zeta)=\bar{D}_{k}^{-p} \mathscr{X}_{j}^{0}(\zeta+p) \bar{D}_{k}^{\mathrm{p}}
$$

Отсюда следует эквивалентность условия $\operatorname{det} \mathscr{X}_{j}^{0}(\zeta) \neq 0$ на $\operatorname{Re} \zeta=\beta_{\tau}-\mathbb{k}$ условию $\operatorname{det} \mathscr{X}_{j}(\zeta) \neq 0$ на $\operatorname{Re} \zeta=\beta_{\tau}-r+1$, кроме того, нули функций $\operatorname{det} \mathscr{X}_{j}(\xi), \operatorname{det} \mathscr{X}_{j}^{0}(\zeta)$ связаны равенством $\zeta=\xi+p$ и имеют одинаковые кратности. Таким образом, справедливость 1) установлена.

Докажем теперь формулу (8.3). Согласно (8.4)-(8.6), (7.21) и (4.9) имеем

$$
\text { ind } \begin{aligned}
\mathscr{A} & =\operatorname{Ind}_{\Gamma} \bar{\aleph}_{0}^{-1} \aleph_{0}-\sum_{j=1}^{|\mathscr{J}|} \operatorname{Ind}_{\beta_{j}-\mathbb{k}} \mathscr{X}_{j}^{0}(\zeta) y_{j}^{-1}(\zeta)-\frac{\ell r^{2}(m-1)}{2}-p|\mathscr{J}| N \\
& +\left\{\operatorname{Ind}_{\Gamma}\left(\sigma_{2} \mathbf{1}-\sigma_{1} \Lambda\right)^{-1}\left(\sigma_{2} \mathbf{1}-\sigma_{1} \bar{\Lambda}\right)-\sum_{j=1}^{|\mathscr{J}|} \operatorname{Ind}_{\beta_{j}-r+1} y_{j}(\zeta+p) y_{j}^{-1}(\zeta)\right\}
\end{aligned}
$$


Легко видеть, что слагаемое в фигурных скобках (обозначим его $\left.I=I_{1}-I_{2}\right)$ в $(8.7)$ целое. Это можно показать следуюшим образом. Рассмотрим задачу Пуанкаpe (7.9), (7.10) с действительными коэффициентами, где $\mathbf{b}_{1}=\mathbf{b}_{1}^{0}, \ldots, \mathbf{b}_{\mathbb{k}+1}=\mathbf{b}_{\mathbb{k}+1}^{0}$, $\mathbf{b}_{\mathrm{k}+2}=\cdots=\mathbf{b}_{r}=\mathbf{0}$ - нулевая матрица, над пространствами с весовым порядком $\beta+p$. Очевидно, что конщевой символ этой задачи есть $\mathscr{X}_{j}^{0}(\zeta)$. Отсюда и из (7.21) следует, что первые три слагаемых справа в (8.7) есть индекс этой задачи.

Итак, $I$ - целое число. Оставаясь цельм, оно непрерьвно зависит от раствора $\vartheta_{\tau}$ криволинейного сектора $Q_{\tau}$ области $Q$ (с вершиной в $\tau$ ). С учетом этого достаточно вычислить $I$ при $\vartheta_{\tau}=\pi$, т.е. для гладкой области. В таком случае легко получим $I_{1}=p r \ell(m-1), I_{2}=p|\mathscr{J}| N$ (см. также [7], [13]). Отсюда и из (8.7) получим (8.3).

Рассмотрим теперь $\mathbb{R}$-линейную задачу $(7.22),(7.23)$, где в краевых условиях $\mathbf{b}_{j}$ заменеы на $\mathbf{b}_{j}^{0}$ и вместо $r$ (там же) имеем $\mathbb{k}+1$. Матрища $G_{0}$ такая же, как выше, но теперь она - квадратная матрища поряка $\ell r$. Аналогично п. $4 \S 7$, исходя из матрицы $G_{0}$, составим концевой символ $\mathscr{X}_{j}^{0}(\zeta)$. Имеет место

Теорема 8.2. Отмеченная задача нётеровая лишь тогда, когда выполнены условия (7.24), (7.25) и на прямой $\operatorname{Re} \zeta=\beta_{j}-\mathbb{k}, \beta_{j}=\beta_{\tau_{j}}$, нет корней определителя концевого символа $\mathscr{X}_{j}^{0}(\zeta), \tau=\tau_{j} \in \mathscr{J}$. При выполнении әтих условий индекс задачи дается формулой

$$
\text { ind } \mathscr{A}=\operatorname{Ind}_{\Gamma} \bar{\aleph}^{-1} \aleph-\sum_{j=1}^{s} \operatorname{Ind}_{\beta_{j}-\mathbb{k}}\left(\mathscr{X}_{j}^{0} y_{j}^{-1}\right)-r \ell(2 \mathbb{k}-r+2)(m-1) \text {. }
$$

Теорема доказывается аналогично теореме 5.1 с привлечением теоремы 8.1. Имеет место

СлЕДСТВИЕ 8.1. Пусть все корни характеристического полинома $P(\lambda)=$ $\Sigma \mathbf{a}_{j}(-\lambda)^{j}$ расположены или в верхней полуплоскости, или в нижней. Тогда в первом случае в формуле (7.26) $=\bar{G}_{0}$, а концевой символ определяется равенством (5.6" $6^{\prime \prime}$, әде $\mathbf{a}=\mathbf{1}, G=G_{0}, \mathbf{b}$ из (7.12). А во втором случае $\aleph=G_{0}$, а концевой символ определяется равенством $\left(5.6^{\prime}\right)$.

По поводу доказательства см. следствие 5.1.

Рассмотрим теперь правильно эллиптические системы. Пусть (8.1), (8.2) краевая задача для правильно эллиптической системы (коэффициенты не обязательно действительные). Аналогично п. $3 \S 7$ построим конщевой символ $\widetilde{\mathscr{X}}_{j}^{0}(\zeta)=$ $\operatorname{diag}\left(\mathscr{X}_{1 j}^{0}(\zeta), \mathscr{X}_{2 j}^{0}(\zeta)\right)$. Справедлива

ТЕОРема 8.3. Задача (8.1), (8.2) для правильно әллиптической системы тогда и только тогда нётеровая, когда выполнены условия (7.19), (7.20) ( с $\mathbf{b}_{j}^{0}$ вместо $\mathbf{b}_{j}$ ) и на прямой $\operatorname{Re} \zeta=\beta_{j}-\mathbb{k}, \quad \beta_{j}=\beta_{\tau_{j}}$, нет корней $\operatorname{det} \widetilde{\mathscr{X}}_{j}^{0}(\zeta)$, $\tau=\tau_{j} \in \mathscr{J}$. При выполнении этих условий индекс задачи дается формулой

$$
\text { ind } \begin{aligned}
\mathscr{A}= & 2^{-1} \sum_{i=1}^{2}\left(\operatorname{Ind}_{\Gamma} \bar{\aleph}_{i}^{-1} \aleph_{i}-\sum_{j=1}^{|\mathscr{J}|} \operatorname{Ind}_{\beta_{j}-\mathbb{k}}\left(X_{i j}^{0}(\zeta) y_{i j}^{-1}(\zeta)\right)\right) \\
- & \frac{r \ell(2 \mathbb{k}-r+2)(m-1)}{2} .
\end{aligned}
$$


2. В этом пункте мы изучим краевые задачи для однородных эллиптических систем, краевые условия которых имеют разные порядки.

Пусть $\ell_{j i}$ - число краевых условий на $\Gamma_{i}, i=1, \ldots,|\mathscr{J}|$, порядка $\mathbb{k}_{j i}, q_{i}$ - число различных порядков краевых условий на $\Gamma_{i}, \sum_{j=1}^{s} \ell_{j i}=r \ell, \mathbb{k}=\max \left\{\mathbb{k}_{j i} \mid j=\right.$ $\left.1, \ldots, q_{i}, \quad i=1, \ldots,|\mathscr{J}|\right\}$.

Рассмотрим сначала $\mathbb{R}$-линейную задачу

$$
\begin{gathered}
A v=f \in X_{(\beta-r)}^{n}(Q), \quad v \in X_{(\beta)}^{n+r}(Q), \\
\operatorname{Re}(B(x, \mathscr{D}) v)=\varphi \in Y_{(\beta)}^{n}(\partial Q \backslash \mathscr{J}),
\end{gathered}
$$

где $B$ - квадратная матрица поряка $\ell r$. Выравним аналогично п. 1 порядки краевых условий (8.11) (при помоши дифференцирования по дуге $\partial / \partial \sigma$ ) так, чтобы они стали равными $\mathbb{k}$. Соответствуюший оператор здесь имеет индекс, равный

$$
\sum_{i=1}^{|\mathscr{F}|} \sum_{j=1}^{q_{i}}\left(\mathbb{k}-\mathbb{k}_{j i}\right) \ell_{j i} .
$$

И представим краевые условия в виде (8.2) (точнее, в виде $\operatorname{Re} B v=\varphi$ ). В результате получим задачу, изученную в теореме 8.3. Следовательно, имеет место

Tеорема 8.4. Задача (8.10), (8.11) тогда и только тогда нётеровая, когда выполнены условия (7.24), (7.25) ( $c G_{0}$ вместо $\left.G\right)$ и на прямой $\operatorname{Re} \zeta=\beta_{j}-\mathbb{k}$, $\beta_{j}=\beta_{\tau_{j}}$, нет корней $\operatorname{det} \mathscr{X}_{j}^{0}(\zeta), \tau=\tau_{j} \in \mathscr{J}$. При выполнении этих условий индекс задачи дается формулой

$$
\text { ind } \begin{aligned}
\mathscr{A}=\operatorname{Ind}_{\Gamma} \bar{\aleph}^{-1} \aleph-\sum_{j=1}^{s} \operatorname{Ind}_{\beta_{j}-\mathbb{k}}\left(\mathscr{X}_{j}^{0}(\zeta) y_{j}^{-1}\right) \\
\\
\quad-\sum_{i=1}^{|\mathscr{J}|} \sum_{j=1}^{q_{i}}\left(\mathbb{k}-\mathbb{k}_{j i}\right) \ell_{j i}-r \ell(2 \mathbb{k}-r+2)(m-1) .
\end{aligned}
$$

Дополним теорему следствием, аналогичным следствию 8.1.

СЛЕДСТВИЕ 8.2. Пусть все корни характеристического полинома $P(\lambda)=$ $\Sigma \mathbf{a}_{j}(-\lambda)^{j}$ расположены или в верхней полуплоскости, или в нижней. Тогда в первом случае в формуле (7.26) $=\bar{G}_{0}$, а концевой символ определяется равенством (5.6"), әде $\mathbf{a}=\mathbf{1}, G=G_{0}, \mathbf{b}$ из (7.12). А во втором случае $\aleph=G_{0}$, а концевой символ определяется равенством $\left(5.6^{\prime}\right)$.

ЗАмЕчАниЕ 8.1. Аналогично [12] легко показать, что краевая задача (8.10), (8.11), не удовлетворяющая условию

$$
\min _{j i}\left\{\mathbb{k}_{j i}\right\} \geqslant \ell^{-1} \max \left\{(\ell r)_{+},(\ell r)_{-}\right\}-1,
$$

не нётеровая. Здесь $(\ell r)_{+}-$число корней (с учетом кратностей) характеристического многочлена из верхней полуплоскости, $(\ell r)_{-}-$из нижней.

Рассмотрим теперь задачу вида (8.10), (8.11) для правильно эллиптических систем. (Здесь в краевых условиях знак "Re" опускаем, $B$ - прямоугольная $(N \times 2 N)$-матрица, $2 N=r \ell$.) Аналогично теореме 5.2, с привлечением теоремы 8.4, получим справедливость следуюшей теоремы. 
Теорема 8.5. Отмеченная задача тогда и только тогда нётеровая, когда выполнены условия (7.13), (7.14) ( $c G_{0}$ вместо $G$ ) и на прямой $\operatorname{Re} \zeta=\beta_{j}-\mathbb{k}$, $\beta_{j}=\beta_{\tau_{j}}$, нет корней $\operatorname{det} \widetilde{\mathscr{X}}_{j}^{0}(\zeta), \tau=\tau_{j} \in \mathscr{J}$. При выполнении этих условий индекс задачи дается формулой

$$
\text { ind } \begin{aligned}
\mathscr{A}= & \sum_{i=1}^{2}\left(\operatorname{Ind}_{\Gamma} \bar{\aleph}_{i}^{-1} \aleph_{i}-\sum_{j=1}^{s} \operatorname{Ind}_{\beta_{j}-\mathbb{k}}\left(\mathscr{X}_{i j}^{0}(\zeta) y_{i j}^{-1}(\zeta)\right)\right) \\
& -\sum_{i=1}^{|\mathscr{J}|} \sum_{j=1}^{q_{i}}\left(\mathbb{k}-\mathbb{k}_{j i}\right) \ell_{j i}-\frac{r \ell(2 \mathbb{k}-r+2)(m-1)}{2} .
\end{aligned}
$$

(Концевой символ $\mathscr{X}_{j}^{0}(\zeta)$ определяется аналогично теореме 7.1.) В случае действительных коэффициентов формула (8.13) упрошается с учетом равенств $\aleph_{2}=$ $\aleph_{1}, \mathscr{X}_{2 j}^{0}(\zeta)=\mathscr{X}_{1 j}^{0}(\zeta)$. (Ситуация такая же, как в теореме 5.2.)

3. Пусть теперь порядок краевых условий $\mathbb{k}$ не меньше $r$. Рассмотрим $\mathbb{R}$-линейную задачу $(8.10),(8.11)$, где $\mathbb{k}=\max \mathbb{k}_{j} \geqslant r$. Пространства, над которыми изучается задача, следующие:

$$
\begin{gathered}
X_{(\gamma)}^{k}(Q)=W_{p,(\gamma)}^{k}(Q) \times \cdots \times W_{p,(\gamma)}^{k}(Q), \\
Y_{\beta, b}^{n}(\partial Q \backslash \mathscr{J})=\prod_{i=1}^{\ell r} W_{p,\left(\beta-b_{i}\right)}^{n-\sigma_{i}-1 / p}(\partial Q \backslash \mathscr{J})
\end{gathered}
$$

или

$$
\begin{aligned}
& X_{(\gamma)}^{k}(Q)=H_{\mu,(\gamma)}^{k}(Q) \times \cdots \times H_{\mu,(\gamma)}^{k}(Q), \\
& Y_{\beta, b}^{n}(\partial Q \backslash \mathscr{J})=\prod_{i=1}^{\ell r} H_{\mu,\left(\beta-b_{i}\right)}^{n-\sigma_{i}}(\partial Q \backslash \mathscr{J}),
\end{aligned}
$$

где $n \geqslant \mathbb{k}+1-r, b_{i}=r+\sigma_{i}, \sigma_{1}=\cdots=\sigma_{\ell_{1}}=\mathbb{k}_{1}, \sigma_{\ell_{1}+1}=\cdots=\sigma_{\ell_{2}}=\mathbb{k}_{2}$, $\sigma_{\ell_{q-1}+1}=\cdots=\sigma_{\ell r}=\mathbb{k}_{q}, \ell_{j}=\max \left\{\ell_{j i}|i=1, \ldots,| \mathscr{J} \mid\right\}, \mathbb{k}_{j}=\max \left\{\mathbb{k}_{j i} \mid i=\right.$ $1, \ldots,|\mathscr{J}|\}$.

Пусть $\mathscr{A}: X_{(\beta)}^{n+r}(Q) \rightarrow X_{(\beta)}^{n}(Q) \times Y_{\beta, b}^{n}(\partial Q \backslash \mathscr{J})$-оператор данной задачи, $\mathscr{A} v=$ $\left(A v, B v_{\left.\right|_{\partial Q \backslash \mathscr{J}}}\right), v \in X_{(\beta)}^{n+r}(Q)$. И пусть $\bar{\partial}-$ оператор задачи Римана-Гильберта для системы Коши-Римана из примера $5.1($ с $k=\ell)$. Обозначим $\mathscr{E}_{V}: V \rightarrow V$ тождественный оператор, где $V$ - любое фиксированное банахово пространство функций на $\partial Q \backslash \mathscr{J}$. Тогда оператор

$$
K_{V}=\left(\bar{\partial}, \mathscr{E}_{V}\right): X_{(\beta-r)}^{n}(Q) \times V \rightarrow X_{(\beta-r-1)}^{n-1}(Q) \times Y_{(\beta-r)}^{n}(\partial Q \backslash \mathscr{J}) \times V
$$

согласно примеру 5.1 тогда и только тогда нётеровый, когда $\beta_{\tau} \neq\left(\pi / \vartheta_{\tau}\right) k+r$, $k$ - любое целое число, $\tau \in \mathscr{J}$. При выполнении этого индекс $K_{V}$ дается равенством (5.16), где $k=\ell$. Если оператор $K_{V} \mathscr{A}$ (в качестве $V$ здесь берем $\left.Y_{\beta, b}^{n}(\partial Q \backslash \mathscr{J})\right)$ нётеровый, то $K_{V}$ и $\mathscr{A}$ нётеровые или не нётеровые одновременно (см. [18, с. 41$\left.]\right)$, и в 
случае нётеровости ind $K_{V} \mathscr{A}=$ ind $K_{V}+$ ind $\mathscr{A}$. Очевидно, что оператор $K_{V} \mathscr{A}$ является оператором краевой задачи вида (8.10), (8.11), при этом система (8.10) порядка $r+1$, а число краевых условий в $(8.11) \ell(r+1)$. Если $\mathbb{k} \geqslant r+1$, с последней задачей поступаем аналогично исходной. В результате получим оператор $K_{V_{1}} K_{V} \mathscr{A}$. Повторяя эти рассуждения, через $\mathbb{k}+1-r$ шагов мы получим оператор $\mathscr{A}_{0}$, из нётеровости которого следует одновременная нётеровость или не нётеровость операторов $\mathscr{A}$ и $K=K_{V_{\mathrm{k}-r}} \ldots K_{V_{1}} K_{V}$. Следовательно, из нётеровости $\mathscr{A}_{0}$ следует нётеровость $\mathscr{A}$ при условии

$$
\beta_{\tau} \neq \frac{\pi}{\vartheta_{\tau}} k+j, \quad j=r, \ldots, \mathbb{k}+1, \quad \tau \in \mathscr{J},
$$

где $k$ - любое целое число. При выполнении последних индексы $\mathscr{A}$ и $\mathscr{A}_{0}$ связаны равенством

$$
\text { ind } \mathscr{A}=\text { ind } \mathscr{A}_{0}+\ell \sum_{j=r}^{\mathbb{k}} \sum_{\tau \in \mathscr{J}}\left\{\left[\left(\beta_{\tau}-j\right) \frac{\vartheta_{\tau}}{\pi}\right]+1\right\}+\ell(\mathbb{k}+1-r)(m-1) \text {. }
$$

(Заметим, что оператор $\mathscr{A}$ может быть нётеровым и для некоторых весовых порядков $\beta=\beta_{\tau}$, для которых не выполнены условия (8.15), но так как при этом оператор $K$ не нётеровый, то и $\mathscr{A}_{0}$ не нётеров.)

Изучим теперь оператор $\mathscr{A}_{0}$. По построению он является оператором следующей краевой задачи:

$$
\begin{aligned}
& \left(\frac{\partial}{\partial x_{1}}+i \frac{\partial}{\partial x_{2}}\right)^{\Delta} A v=f \in X_{(\beta-\mathbb{k}-1)}^{n-\Delta}(Q), \quad v \in X_{(\beta)}^{n+r}(Q), \\
& \operatorname{Re}(B v)=\varphi \in Y_{\beta, b}^{n}(\partial Q \backslash \mathscr{J}), \\
& \operatorname{Re}(A v)=\varphi_{0} \in Y_{(\beta-r)}^{n}(\partial Q \backslash \mathscr{J}), \\
& \ldots \ldots \ldots \ldots \ldots \ldots \ldots \ldots \ldots \ldots \ldots \ldots \ldots \ldots \ldots \ldots \ldots \ldots \ldots \ldots \ldots \ldots \ldots \ldots \\
& \operatorname{Re}\left(\left(\frac{\partial}{\partial x_{1}}+i \frac{\partial}{\partial x_{2}}\right)^{\mathbb{k}-r} A v\right)=\varphi_{\mathbb{k}-r} \in Y_{(\beta-\mathbb{k})}^{n}(\partial Q \backslash \mathscr{J}),
\end{aligned}
$$

где $\Delta=\mathbb{k}+1-r$. Система (8.17) - однородная эллиптическая система порядка $\mathbb{k}+1$. Краевые условия имеют порядок $\mathbb{k}$, число их $(\mathbb{k}+1) \ell$. Такие задачи изучены в теоремах 7.3 и 8.4. В (8.18) мы имеем $\ell_{j i}$ условий порядка $\mathbb{k}_{j i}, j=1, \ldots, q_{i}\left(\right.$ на $\left.\Gamma_{i}\right)$, $\ell$ условий порядка $r, \ell$ условий порядка $r+1$ и т.д., $\ell$ условий порядка $\mathbb{k}$ (последние из условий (8.18)). В соответствии с теоремой 8.4 выравним порядки краевых условий (оператором дифференцирования по дуге) так, чтобы они стали равными $\mathbb{k}$. Представим систему и полученные краевые условия в виде (7.22), (7.23). Пусть $\mathbf{b}$ - матрица коэффициентов при $\partial / \partial x_{2}$ и $G=\left(\mathbf{b}_{1}, \ldots, \mathbf{b}_{\mathbb{k}+1}\right)$. Разложим $\mathbf{b}$ по формуле (2.1): $\mathbf{b}=T \operatorname{diag}\left(\Lambda_{1}, \Lambda_{2}\right) T^{-1}, T=\left(T_{1}, T_{2}\right)$. И пусть

$$
\begin{aligned}
& \operatorname{det} \aleph \neq 0 \quad \text { всюду на } \quad \partial Q \backslash \mathscr{J}, \\
& \operatorname{det} \aleph(\tau \pm 0) \neq 0, \quad \tau \in \mathscr{J}, \\
& \operatorname{det} \mathscr{X}_{j}(\zeta) \neq 0 \quad \text { на прямой } \quad \operatorname{Re} \zeta=\beta_{j}-\mathbb{k}, \quad \tau_{j} \in \mathscr{J},
\end{aligned}
$$


где $\aleph=\left(\bar{G}_{1}, G T_{2}\right), \mathscr{X}_{j}(\zeta)-$ концевой символ. Условия (8.19)-(8.21) необходимы и достаточны для нётеровости задачи (8.17), (8.18). Отсюда и из формул (8.14) (где $r=\mathbb{k}+1),(8.16)$ получим, что справедлива

ТЕОРема 8.6. Пусть выполнены условия (8.15). Для нётеровости задачи (8.10), (8.11) $c \mathbb{k} \geqslant r$ необходимо и достаточно выполнения условий (8.19)-(8.21). При выполнении этих условий индекс задачи дается равенством

$$
\text { ind } \begin{aligned}
\mathscr{A}=\operatorname{Ind}_{\Gamma} \bar{\aleph}^{-1} \aleph-\sum_{j=1}^{|\mathscr{J}|} \operatorname{Ind}_{\beta_{j}-\mathbb{k}}\left(\mathscr{X}_{j}(\zeta) y_{j}^{-1}(\zeta)\right) \\
+\ell \sum_{j=r}^{\mathbb{k}} \sum_{\tau \in \mathscr{J}}\left\{\left[\left(\beta_{\tau}-j\right) \frac{\vartheta_{\tau}}{\pi}\right]+1\right\}-\sum_{i=1}^{\mid \mathscr{J}} \sum_{j=1}^{q_{i}}\left(\mathbb{k}-\mathbb{k}_{j i}\right) \ell_{j i} \\
-\ell|\mathscr{J}| \frac{(\mathbb{k}-r+1)(\mathbb{k}+r)}{2}-\ell\left(\mathbb{k}^{2}+\mathbb{k}+r\right)(m-1),
\end{aligned}
$$

где $[a]$ - целая часть числа а. (Остальные обозначения такие же, как $\boldsymbol{\theta}(8.14)$.)

\section{Справедливо также}

СлЕДСТВИЕ 8.3. Пусть все корни характеристического полинома $P(\lambda)=$ $\Sigma \mathbf{a}_{j}(-\lambda)^{j}$ расположены или в верхней полуплоскости, или в нижсней. Тогда в первом случае в формуле $(8.22) \aleph=\bar{G}$, а кончевой символ определяется равенством $\left(5.6^{\prime \prime}\right)$, дее $\mathbf{a}=\mathbf{1}, \mathbf{b}$ из (7.12) (соответствующей (8.17)). Во втором случае $=G$, а кончевой символ определяется равенством $\left(5.6^{\prime}\right)$, əде $\mathbf{a}=\mathbf{1}$, а b есть матрииа (7.12), которая соответствует (8.17), (8.18), где коэффициенты $A$ заменены на комплексно сопряженные. ( $C$ учетом последнего определяется и матрица $G$.)

ДокАЗАТЕльство. Первая часть есть следствие теоремы и разложения (2.1), если учесть, что все собственные значения матрицы (7.12), соответствующей (8.17), расположены выше прямой $\operatorname{Im} \lambda=0$, так как характеристический полином последней есть $\Delta(\lambda)=(i-\lambda)^{\mathbb{k}-r+1} P(\lambda)$.

Рассмотрим теперь второй случай. Заменим каждое уравнение системы (8.10) и каждое краевое условие под знаком "Re" на комплексно сопряженное. Затем обозначим $\bar{v}$, где $v$ искомая, снова через $v$. Эти преобразования не влияют на нётеровость и индекс. В результате мы получим разобранный случай. Откуда следует требуемое.

Переход от $\mathbb{R}$-линейных задач к задачам для правильно эллиптических систем пояснялся в теореме 5.2. С учетом этого получим следующую формулу для индек- 
са в случае правильно эллиптических систем:

$$
\text { ind } \begin{aligned}
\mathscr{A}= & 2^{-1} \sum_{i=1}^{2}\left(\operatorname{Ind}_{\Gamma} \bar{\aleph}_{i}^{-1} \aleph_{i}-\sum_{j=1}^{|\mathscr{J}|} \operatorname{Ind}_{\beta_{j}-\mathbb{k}}\left(\mathscr{X}_{i j}(\zeta) y_{i j}^{-1}(\zeta)\right)\right) \\
& +\ell \sum_{j=r}^{\mathbb{k}} \sum_{\tau \in \mathscr{J}}\left\{\left[\left(\beta_{\tau}-j\right) \frac{\vartheta_{\tau}}{\pi}\right]+1\right\}-\sum_{i=1}^{|\mathscr{J}|} \sum_{j=1}^{q_{i}}\left(\mathbb{k}-\mathbb{k}_{j i}\right) \ell_{j i} \\
& -\ell|\mathscr{J}| \frac{(\mathbb{k}-r+1)(\mathbb{k}+r)}{2}-\frac{\ell\left(\mathbb{k}^{2}+\mathbb{k}+r\right)(m-1)}{2},
\end{aligned}
$$

где $\aleph_{1}=\bar{G} \bar{T}_{1}, \aleph_{2}=G T_{2}$.

Условия, необходимые и достаточные для нётеровости задачи, опускаем, с этим несложно разобраться. Они аналогичны условиям (7.13), (7.14). Весовой порядок, кроме условия, сформированного в начале параграфа, должен подчиняться (8.15).

В случае действительных коэффициентов в $(8.23) \aleph_{2}=\aleph_{1}=G T_{1}, \mathscr{X}_{2 j}=\mathscr{X}_{1 j}$.

4. В этом пункте дается применение предыдущих формул для индекса к задаче Дирихле. Согласно нашим утверждениям индекс задачи достаточно найти хотя бы для одного значения весового порядка $\beta=\left\{\beta_{\tau}\right\}$. (Тогда индекс для произвольного $\beta$ получаем с учетом нулей конщевого символа.) Наибольшие трудности здесь возникают при вычислении $\operatorname{Ind}_{\beta_{\tau}} \mathscr{X}_{j}(\zeta) y_{j}^{-1}(\zeta)$. В некоторых случаях эти затруднения можно преодолеть [7]. Сформулируем одно утверждение из [7] в удобной для нас форме.

ЛЕмма 8.1. Пусть в (4.12) $C\left(\tau_{j}-0\right)=C\left(\tau_{j}+0\right)$. Тогда

$$
\operatorname{Ind}_{\varepsilon} \mathscr{X}_{j}(\zeta) y_{j}^{-1}(\zeta)=2^{-1}\left(k-k_{j}(0)\right)-\sum_{\varrho>0} k_{j}(i \varrho),
$$

где $\varepsilon>0$ - любое фиксированное число такое, что в "полосе" $0<|\operatorname{Re} \zeta| \leqslant \varepsilon$ нет нулей $\operatorname{det} \mathscr{X}_{j}(\zeta), \quad k$ - порядок матрич $\mathbf{a}, \mathbf{b} и з(4.13), k_{j}(i \varrho)=0$, если $\zeta=i \varrho$ не есть нуль функции $\operatorname{det} \mathscr{X}_{j}(\zeta)$, и $k_{j}(i \varrho)$ равняется кратности нуля в противном случае.

ПримеР 8.1. Рассмотрим задачу Дирихле для эллиптической системы (7.9) $\ell$ уравнений порядка $r=2 r^{\prime}, r^{\prime} \in N$, с действительными коэффициентами

$$
\begin{gathered}
\sum_{j=1}^{r+1} \mathbf{a}_{j}\left(\frac{\partial}{\partial x_{2}}\right)^{r-j+1}\left(\frac{\partial}{\partial x_{1}}\right)^{j-1} u=f \quad \text { при } \quad x \in Q, \\
\frac{\partial^{i} u}{\partial \nu^{i}}=\varphi_{i}, \quad i=0, \ldots, r / 2-1, \quad \text { на } \quad \partial Q \backslash \mathscr{J},
\end{gathered}
$$

где $\nu$ - нормаль к границе, $\mathbf{a}_{j} \in C^{\infty}(\bar{Q})$ - квадратные матрицы порядка $\ell$. Требуется найти условия, необходимые и достаточные для нётеровости задачи, и формулу для индекса. 
Задачу изучим, следуя п. 2. Составим матрицу (7.12) и разложим ее по формуле (2.1): $\mathbf{b}=T \operatorname{diag}(\Lambda, \bar{\Lambda}) T^{-1}, T=\left(T_{1}, \bar{T}_{1}\right)$. Обозначим через $\mathfrak{U}$ квадратную матрицу порядка $r \ell / 2$, составленную из первых $r \ell / 2$ строк матрицы $\mathfrak{U}$.

Выравним порядки краевых условий при помощи дифференцирований по дуге $\partial / \partial \sigma:$

$$
\left(\frac{\partial}{\partial \sigma}\right)^{r / 2-1-i}\left(\frac{\partial}{\partial \nu}\right)^{i}=\psi_{j}, \quad j, i=0, \ldots, r / 2-1,
$$

представим полученные условия в виде (8.2) и составим матрищу

$$
G_{0}=\left(\mathbf{b}_{1}^{0}, \ldots, \mathbf{b}_{r^{\prime}}^{0}, \mathbf{0}, \ldots, \mathbf{0}\right),
$$

где нулевые блоки $\mathbf{0}$ расположены на последних $r^{\prime}$ местах. И пусть $\mathfrak{X}=$ $\left(\mathbf{b}_{1}^{0}, \ldots, \mathbf{b}_{r^{\prime}}^{0}\right)$. Легко видеть, что $\mathfrak{X}$ - невырожденная матрица всюду на $\partial Q \backslash \mathscr{J}$, имеющая такие же односторонние пределы в точках $\mathscr{J}$. С учетом этого в формуле для концевого символа имеем $C\left(\tau_{j} \pm 0\right)=\mathfrak{U}^{-1} \mathfrak{U}$ (т.е. мы находимся в условиях леммы 8.1). Отсюда же следует, что условиями, необходимыми и достаточными для нётеровости задачи Дирихле, будут условия

$$
\begin{aligned}
\operatorname{det} \mathfrak{U} \neq 0 \quad \text { всюду на } \quad \partial Q, \\
\operatorname{det} \mathscr{X}_{j}^{0}(\zeta) \neq 0 \quad \text { при } \quad \operatorname{Re} \zeta=\beta_{\tau_{j}}-\frac{r}{2}+1 .
\end{aligned}
$$

(Заметим, что $\mathfrak{U}$ непрерывна в $\bar{Q}$.) Индекс задачи (согласно (8.13), где $\mathbb{k}=r / 2-1$, и (8.24) с $k=r \ell / 2)$ определяется равенством

$$
\text { ind } \mathscr{A}=\operatorname{Ind}_{\Gamma} \overline{\mathfrak{U}}^{-1} \mathfrak{U}-r \ell|\mathscr{J}| \frac{r(r-2)}{8}-\sum_{j=1}^{|\mathscr{J}|}\left\{\frac{1}{2}\left(k-k_{j}(0)\right)-\sum_{\varrho>0} k_{j}(i \varrho)-S_{j}\right\} \text {, }
$$

где $\pm S_{j}$ равен числу нулей (включая кратности) определителя концевого символа $\operatorname{det} \mathscr{X}_{j}^{0}(\zeta)$ между прямыми $\operatorname{Re} \zeta=-\varepsilon, \operatorname{Re} \zeta=\beta_{\tau}-r / 2+1, \tau=\tau_{j} \in \mathscr{J}$. (Знак "+", когда $\beta_{\tau} \geqslant r / 2-1-\varepsilon$, знак “-”, когда $\beta_{\tau} \leqslant r / 2-1-\varepsilon$.)

\section{§9. Формула индекса для общих эллиптических систем на плоскости}

1. Изучим сначала $\mathbb{R}$-линейную задачу (6.3):

$$
\begin{gathered}
\mathscr{L}(x, \mathscr{D}) v=f \in \mathfrak{B}(Q), \quad v \in \mathfrak{A}(Q), \\
\operatorname{Re}(\mathscr{B}(x, \mathscr{D}) v)=\varphi \in \mathfrak{C}(\partial Q \backslash \mathscr{J}) .
\end{gathered}
$$

Предполагаем, что выполнены условия 1)-3) $\S 6$, причем в 1) $n$ необязательно четное число. Пусть $\Gamma_{j}, j=1, \ldots,|\mathscr{J}|,-$ гладкие дуги, составляющие границу области $Q$. В дальнейшем предполагаем, что порядок отличного от нуля элемента $b_{i l}(x, \mathscr{D})$ на $\Gamma_{j}$ матрицы $\mathscr{B}(x, \mathscr{D})$ дается равенством

$$
\operatorname{ord} b_{i l}(x, \mathscr{D})=\sigma_{i j}+t_{l}, \quad j=1, \ldots,|\mathscr{J}|, \quad i=1, \ldots, n, \quad l=1, \ldots, \ell .
$$


Требуется определить формулу для индекса задачи (9.1).

Пространства $\mathfrak{A}(Q), \mathfrak{B}(Q), \mathfrak{C}(\partial Q \backslash \mathscr{J})$, когда задача $(9.1),(9.2)$ рассматривается над весовыми пространствами Соболева, являются конечномерными расширениями пространств (6.4):

$$
\begin{gathered}
\mathfrak{A}(Q)=\prod_{i=1}^{\ell} W_{p,\left(\beta+t_{i}-t_{0}\right)}^{n+t_{i}}(Q), \quad \mathfrak{B}(Q)=\prod_{i=1}^{\ell} W_{p,\left(\beta-s_{i}-t_{0}\right)}^{n-s_{i}}(Q), \\
\mathfrak{C}(\partial Q \backslash \mathscr{J})=\prod_{i=1}^{N} W_{p,\left(\beta-\sigma_{i}-t_{0}\right)}^{n-\sigma_{i}-1 / p}(\partial Q \backslash \mathscr{J}),
\end{gathered}
$$

а в случае пространств Гёльдера

$$
\begin{gathered}
\mathfrak{A}(Q)=\prod_{i=1}^{\ell} H_{\mu,\left(\beta+t_{i}-t_{0}\right)}^{n+t_{i}}(Q), \quad \mathfrak{B}(Q)=\prod_{i=1}^{\ell} H_{\mu,\left(\beta-s_{i}-t_{0}\right)}^{n-s_{i}}(Q), \\
\mathfrak{C}(\partial Q \backslash \mathscr{J})=\prod_{i=1}^{N} H_{\mu,\left(\beta-\sigma_{i}-t_{0}\right)}^{n-\sigma_{i}}(\partial Q \backslash \mathscr{J}),
\end{gathered}
$$

где $t_{0}=\max \left\{t_{j} \mid j=1, \ldots, \ell\right\}, n \geqslant 0$ - неотрицательное целое число такое, что $n>\sigma_{0}=\max \left\{\sigma_{i j}|i=1, \ldots, n, j=1, \ldots,| \mathscr{J} \mid\right\}$ в $(9.4)$ и $n \geqslant \max \left\{0, \sigma_{0}\right\}$ в (9.5). Мы будем пользоваться и следующими обозначениями: $\mathfrak{A}\left(n+t_{0} ; Q\right)$ есть пространство $\mathfrak{A}(Q)$, где все $t_{i}$ равны $t_{0} ; \mathfrak{B}(n ; Q)$ есть пространство $\mathfrak{B}(Q)$, где все $s_{i}=0 ; \mathfrak{C}\left(n, n_{1}, \ldots, n_{k} ; \partial Q \backslash \mathscr{J}\right)$ есть пространство $\mathfrak{C}(\partial Q \backslash \mathscr{J})$, где $n=k, \sigma_{i}=n_{i}$.

Задача (9.1), (9.2), если подействовать на уравнения некоторыми степенями оператора Коши-Римана и произвести замену искомых посредством таких же операторов, сводится к задаче (7.1), (7.2) для однородной эллиптической системы (7.1). Для обоснования этого нам потребуются операторы $\mathfrak{L}$ и $\mathfrak{N}$. Опишем их. Пусть $\bar{\partial}-$ оператор задачи Римана-Гильберта из примера 5.1 для случая одного уравнения. Рассмотрим оператор $\mathfrak{L}_{1}=\left(\bar{\partial}^{t_{0}-t_{1}}, \ldots, \bar{\partial}^{t_{0}-t_{k}}\right)$ (при $t_{0}=t_{j}$ считаем, что $\bar{\partial}^{0}=\mathscr{E}-$ тождественный оператор), определенный на пространстве $\mathfrak{A}\left(r+t_{0} ; Q\right)$. При этом для $v=\left(v_{1}, \ldots, v_{k}\right) \in \mathfrak{A}\left(r+t_{0} ; Q\right)$ имеем

$$
\mathfrak{L}_{1} v=\left(\bar{\partial}^{t_{0}-t_{1}} v_{1}, \ldots, \bar{\partial}^{t_{0}-t_{k}} v_{k}\right) \equiv\left(u_{1}, \ldots, u_{k}\right)=u,
$$

где $u_{j}$ при $t_{j} \neq t_{0}$ представляется в виде

$$
u_{j}=\left(\partial_{\bar{z}}^{t_{0}-t_{j}} v_{j},\left.\operatorname{Re} v_{j}\right|_{\partial Q},\left.\operatorname{Re}\left(\partial_{\bar{z}} v_{j}\right)\right|_{\partial Q}, \ldots,\left.\operatorname{Re}\left(\partial_{\bar{z}}^{t_{0}-t_{j}-1} v_{j}\right)\right|_{\partial Q}\right) .
$$

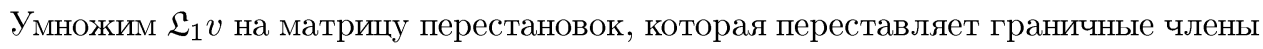
в конец вектора $u$ так, что полученная при этом вектор-функция $w$ принадлежит $\mathfrak{W}=\mathfrak{A}(Q) \times V_{1}(\partial Q \backslash \mathscr{J})$, где

$$
V_{1}=V_{1}(\partial Q \backslash \mathscr{J})=\prod_{j=1}^{k} \mathfrak{C}\left(r+t_{0}, 0,1, \ldots, t_{0}-t_{j}-1 ; \partial Q \backslash \mathscr{J}\right)
$$


(здесь в произведении опущены множители с $t_{j}=t_{0}=\max \left\{t_{j} \mid j=1, \ldots, \ell\right\}$ ). Полученньй таким образом оператор, действующий из $\mathfrak{A}\left(n+t_{0} ; Q\right)$ в $\mathfrak{W}$, обозначим $\mathfrak{L}$.

Пусть $\mathfrak{N}_{1}$ - оператор, определенньй аналогично $\mathfrak{L}$, но теперь вместо $t_{0}-t_{j}$ берем $-s_{j}$ и $\mathfrak{N}_{1}: \mathfrak{B}(Q) \rightarrow \mathfrak{B}(n ; Q) \times V_{2}(\partial Q \backslash \mathscr{J})$, где

$$
V_{2}=V_{2}(\partial Q \backslash \mathscr{J})=\prod_{j=1}^{k} \mathfrak{C}\left(r,-1,-2, \ldots, s_{j} ; \partial Q \backslash \mathscr{J}\right)
$$

(Здесь пропушены множители, для которых $s_{j}=0$.) Тогда $\mathfrak{N}=\left(\mathfrak{N}_{1}, \mathscr{E}\right)$, где $\mathscr{E}-$ тождественный оператор, действуюший в пространстве $V=\mathfrak{C}(\partial Q \backslash \mathscr{J}) \times V_{1}(\partial Q \backslash$ $\mathscr{J})$. Следовательно, $\mathfrak{N}$ отображает $\mathfrak{B}(Q) \times V$ в $\mathfrak{B}(n ; Q) \times V$. Операторы $\mathfrak{L}$ и $\mathfrak{N}$ согласно примеру 5.1 тогда и только тогда нётеровые, когда выполнены условия

$$
\begin{aligned}
& \beta_{\tau} \neq \frac{\pi}{\vartheta_{\tau}} k+j_{i}, \quad j_{i}=t_{0}+s_{i}, \ldots, t_{0}-1, \quad i=1, \ldots, \ell, \quad \text { при } \quad s_{i}<0, \\
& \beta_{\tau} \neq \frac{\pi}{\vartheta_{\tau}} n+j_{i}, \quad j_{i}=0,1, \ldots, t_{0}-t_{i}-1, \quad i=1, \ldots, \ell, \quad \text { при } \quad t_{i}<t_{0},
\end{aligned}
$$

где $k, n$ - любые целые, и их индексы согласно (5.16) с $k=1$ даются формулами

$$
\begin{aligned}
& \text { ind } \mathfrak{L}=-\sum_{j=1}^{\ell}\left(t_{0}-t_{j}\right)\left\{m-1+\sum_{i=0}^{t_{0}-t_{j}} \sum_{\tau \in \mathscr{J}}\left\{\left[\left(\beta_{\tau}-t_{0}+t_{j}+i\right) \frac{\vartheta_{\tau}}{\pi}\right]+1\right\}\right\} \text {, } \\
& \text { ind } \mathfrak{N}=-\sum_{j=1}^{\ell}\left(-s_{j}\right)\left\{m-1+\sum_{i=0}^{-s_{j}-1} \sum_{\tau \in \mathscr{J}}\left\{\left[\left(\beta_{\tau}-t_{0}-s_{j}-i\right) \frac{\vartheta_{\tau}}{\pi}\right]+1\right\}\right\} \text {, }
\end{aligned}
$$

где $[a]$ - целая часть числа $a$. Пусть теперь $\mathscr{A}: \mathfrak{A}(Q) \rightarrow \mathfrak{B}(Q) \times \mathfrak{C}(\partial Q \backslash \mathscr{J})-$ оператор задачи $(9.1),(9.2)$, и пусть $\mathscr{E}$ - тождественньй оператор, действуюший в $V_{1}(\partial Q \backslash \mathscr{J}) ; \mathscr{A}_{1}=(\mathscr{A}, \mathscr{E})$ - оператор, действующий из $\mathfrak{A}\left(n+t_{0} ; Q\right) \times V_{1}(\partial Q \backslash \mathscr{J})$ в $\mathfrak{B}(Q) \times \mathfrak{C}(\partial Q \backslash \mathscr{J}) \times V_{1}(\partial Q \backslash \mathscr{J})$. Рассмотрим оператор

$$
\mathscr{A}_{0}=\mathfrak{N} \mathscr{A}_{1} \mathfrak{L}: \mathfrak{A}\left(r+t_{0} ; Q\right) \rightarrow \mathfrak{B}(n ; Q) \times V_{2}(\partial Q \backslash \mathscr{J}) \times \mathfrak{C}(\partial Q \backslash \mathscr{J}) \times V_{1}(\partial Q \backslash \mathscr{J}), \quad(9.10)
$$

где $V_{1}$ и $V_{2}$ из $(9.6),(9.7)$. Так как $\mathfrak{L}$ и $\mathfrak{N}$ - нётеровые операторы лишь тогда, когда выполнены условия (9.8), то при выполнении последних $\mathscr{A}_{0}$ и $\mathscr{A}_{1}$ нётеровые или не нётеровые одновременно. Далее, из нётеровости $\mathscr{A}_{1}$, очевидно, следует нётеровость $\mathscr{A}$, и обратно, причем ind $\mathscr{A}_{1}=$ ind $\mathscr{A}$. Поэтому $\mathscr{A}_{0}$ и $\mathscr{A}$ нётеровые или не нётеровые одновременно. И в случае нётеровости с учетом (9.10) имеем

$$
\text { ind } \mathscr{A}=\text { ind } \mathscr{A}_{0}-\text { ind } \mathfrak{L}-\text { ind } \mathfrak{N} \text {. }
$$


Изучим теперь оператор $\mathscr{A}_{0}$. Легко видеть, что он есть оператор следуюшей краевой задачи:

$$
\begin{gathered}
\mathscr{U}(\mathscr{D}) \mathscr{L}(x, \mathscr{D}) \mathscr{T}(\mathscr{D}) v=f \in \mathfrak{B}(n ; Q), \\
\operatorname{Re}\left(\partial_{\bar{z}}^{i-1} v_{j}\right)=\varphi_{i j} \in \mathfrak{C}\left(n+t_{0}, i-1 ; \partial Q \backslash \mathscr{J}\right), \\
j=1, \ldots, \ell, \quad i=1, \ldots, t_{0}-t_{j}, \\
\operatorname{Re}(\mathscr{B}(x, \mathscr{D}) \mathscr{T}(\mathscr{D}) v)=\varphi \in \mathfrak{C}(\partial Q \backslash \mathscr{J}), \\
\operatorname{Re}\left(\partial_{\bar{z}}^{j-1} \sum_{q=1}^{\ell} a_{i q}(x, \mathscr{D}) \partial_{\bar{z}}^{t_{0}-t_{q}} v_{q}\right)=\psi_{i j} \in \mathfrak{C}(n,-j ; \partial Q \backslash \mathscr{J}), \\
j=1, \ldots,-s_{i}, \quad i=1, \ldots, \ell, \quad v \in \mathfrak{A}\left(n+t_{0} ; Q\right),
\end{gathered}
$$

где $\left\{a_{i j}\right\}=\mathscr{L}, \mathscr{U}(\mathscr{D})$ и $\mathscr{T}(\mathscr{D})$ - диагональные матрицы, определенные равенствами

$$
\mathscr{U}(\mathscr{D})=\operatorname{diag}\left(\partial_{\bar{z}}^{-s_{1}}, \ldots, \partial_{\bar{z}}^{-s_{\ell}}\right), \quad \mathscr{T}(\mathscr{D})=\operatorname{diag}\left(\partial_{\bar{z}}^{t_{0}-t_{1}}, \ldots, \partial_{\bar{z}}^{t_{0}-t_{\ell}}\right)
$$

Считаем, что $\partial_{\bar{z}}^{0}$ есть единица. Отметим, что в первой группе краевых условий (9.13) отсутствуют члены, для которых $t_{0}-t_{j}=0$, а в 3 -й-для которых $s_{i}=0$.

Для системы (9.12), очевидно, имеем

$$
\operatorname{det}\left(\mathscr{U}(\xi) \mathscr{L}_{0}(x, \xi) \mathscr{T}(\xi)\right)=\left(\xi_{1}+i \xi_{2}\right)^{\ell t_{0}-N} \operatorname{det} \mathscr{L}_{0}(x, \xi)
$$

Поэтому согласно (6.2) она эллиптическая. Заметим, что матрица $\mathscr{T}$ выравнивает порядки столбцов матрицы $\mathscr{L}$, а $\mathscr{U}$ - порядки строк, и они становятся равными $t_{0}$. (Под порядком строки или столбца понимаем наибольший из порядков элементов.) Заметим также, что число краевых условий (9.13) равно $k t_{0}$ и пространства $\mathfrak{A}\left(n+t_{0} ; Q\right), \mathfrak{A}(n ; Q)$ совпадают с пространствами $X_{(\beta)}^{n+t_{0}}(Q), X_{\left(\beta-t_{0}\right)}^{n}(Q)$ соответственно из $\S 7,8$. Таким образом, (9.12), (9.13) - эллиптическая задача, изученная в предыдущих параграфах.

Пусть $k$ - максимальный из порядков граничных условий (9.13). Возможны два случая: а) $k=t_{0}-1$; b) $k=t_{0}+\sigma_{0}, \sigma_{0} \geqslant 0$, так как третье из краевых условий порядка $t_{0}-1$, а второе $t_{0}+\sigma_{0}$. (Предполагаем, что хотя бы одно из чисел $s_{j}$ отлично от нуля или что хотя бы одно $t_{j} \neq t_{0}$. Противоположный случай изучен в предыдущих параграфах.) Рассмотрим оба случая по отдельности.

a) При $k=t_{0}-1$ следуем п. $2 \S 8$. Выравним порядки всех граничных условий до $t_{0}-1$ при помощи дифференцирований по дуге. Соответствуюший этому оператор имеет следуюший индекс:

$$
\varkappa=2^{-1}|\mathscr{J}| \sum_{j=1}^{\ell}\left\{s_{j}\left(s_{j}+1\right)+\left(t_{0}-t_{j}\right)\left(t_{0}+t_{j}-1\right)\right\}-\sum_{j=1}^{|\mathscr{I}|} \sum_{i=1}^{N}\left(\sigma_{i j}+1\right) .
$$


(Ввиду $(9.3),(9.13) i$-я строка матрицы $\mathscr{B}(x, \mathscr{D}) \mathscr{T}(\mathscr{D})$ имеет порядок, равньй $t_{0}+\sigma_{i j}$ на $\left.\Gamma_{j}.\right)$ Далее, представим задачу в виде $(7.22),(7.23):$

$$
\begin{gathered}
\sum_{j=1}^{t_{0}+1} \mathbf{a}_{j}\left(\frac{\partial}{\partial x_{2}}\right)^{t_{0}-j+1}\left(\frac{\partial}{\partial x_{1}}\right)^{j-1} v=f \in X_{\left(\beta-t_{0}\right)}^{n}(Q), \quad v \in X_{(\beta)}^{n+t_{0}}(Q), \\
\operatorname{Re} B v \equiv \operatorname{Re}\left(\sum_{j=1}^{t_{0}} \mathbf{b}_{j}\left(\frac{\partial}{\partial x_{2}}\right)^{t_{0}-j}\left(\frac{\partial}{\partial x_{1}}\right)^{j-1} v\right)=\varphi \in Y_{(\beta)}^{n}(\partial Q \backslash \mathscr{J}),
\end{gathered}
$$

где $\mathbf{a}_{1}, \ldots, \mathbf{a}_{t_{0}+1}-$ квадратные матрицы порядка $\ell, \mathbf{b}_{1}, \ldots, \mathbf{b}_{t_{0}}-\left(t_{0} \times \ell\right)$-матрицы. Определим матрищу $\mathbf{b}$ формулой (7.12). Разложим последнюю по формуле (2.1): $\mathbf{b}=T \operatorname{diag}\left(\Lambda_{1}, \Lambda_{2}\right) T^{-1}, T=\left(T_{1}, T_{2}\right)$. Пусть

$$
\begin{array}{cc}
\operatorname{det} \aleph \neq 0 \quad \text { всюду на } & \partial Q \backslash \mathscr{J}, \\
\operatorname{det} \aleph(\tau \pm 0) \neq 0, \quad \tau \in \mathscr{J},
\end{array}
$$

где $\aleph=\left(\bar{G} \bar{T}_{1}, G T_{2}\right), G=\left(\mathbf{b}_{1}, \ldots, \mathbf{b}_{t_{0}}\right)$. Задаче $(9.15),(9.16)$ поставим в соответствие задачу Римана-Гильберта для эллиптической системы $k=\ell t_{0}$ уравнений $\mathscr{D}_{1} u+\mathbf{b} \mathscr{D}_{2} u=g, \operatorname{Re}(G u)=\psi$, где $\mathbf{b}$ определена формулой (7.12).

Пусть $\mathscr{X}_{j}(\zeta), \tau_{j}=\tau \in \mathscr{J},-$ конщевой символ этой задачи (он определен в п. $1 \S 5$; см. (5.6)-(5.8), (5.6'), (5.6 $\left.\left.{ }^{\prime \prime}\right)\right)$, и пусть

$$
\beta_{\tau}-t_{0}+1 \notin \mathbb{N} \cup\{0\}, \quad \tau \in \mathscr{J},
$$

где $\mathbb{N}$ - множество натуральных чисел. Тогда согласно теореме 7.3 задача (9.15), (9.16) тогда и только тогда нётеровая, когда выполнены $(9.17),(9.18)$ и условие:

$$
\operatorname{det} \mathscr{X}_{j}(\zeta) \neq 0 \quad \text { на прямой } \quad \operatorname{Re} \zeta=\beta_{\tau}-t_{0}+1, \quad \tau=\tau_{j} \in \mathscr{J} .
$$

При выполнении этих условий индекс задачи дается равенством

$$
\varkappa_{1}=\operatorname{Ind}_{\Gamma} \bar{\aleph}^{-1} \aleph-\sum_{j=1}^{|\mathscr{J}|} \operatorname{Ind}_{\beta_{j}-t_{0}+1} \mathscr{X}_{j}(\zeta) y_{j}^{-1}-\ell t_{0}^{2}(m-1) .
$$

Индексы задачи (9.12), (9.13) и (9.15), (9.16) связаны равенством

$$
\varkappa_{1}=\operatorname{ind} \mathscr{A}_{0}+\varkappa \text {, }
$$

где $\varkappa$ из (9.14). Таким образом, с учетом вышеизложенного и формул $(9.11),(9.9)$, (9.21) $(9.22)$, получим, что справедлива следующая 
TеОРема 9.1. Пусть выполнены условия (9.8), (9.19), и пусть $\sigma_{0}=$ $\max \left\{\sigma_{i j}\right\} \leqslant-1$ (см. (9.3)). Для нётеровости задачи (9.1), (9.2) необходимо и достаточно выполнения условий (9.17), (9.18), (9.20). При выполнении этих условий индекс задачи дается формулой

$$
\text { ind } \mathscr{A}=\varkappa_{1}-\varkappa-\text { ind } \mathfrak{L}-\text { ind } \mathfrak{N}
$$

где слагаемые справа определены формулами (9.9), (9.14), (9.22).

Пусть $\beta=\left\{\beta_{\tau}\right\}, \quad \gamma=\left\{\gamma_{\tau}\right\}-$ два весовых порядка, для которых выполнены условия (9.8), (9.17)-(9.19), и пусть $\mathscr{A}_{\beta}, \mathscr{A}_{\gamma}$ - операторь, соответствующие задаче (9.1), (9.2) при $\beta=\beta$ и $\beta=\gamma$. Тогда индексы $\mathscr{A}_{\beta}$ и $\mathscr{A}_{\gamma}$ связань равенством

$$
\text { ind } \mathscr{A}_{\gamma}-\text { ind } \mathscr{A}_{\beta}=\sum_{\tau \in \mathscr{J}} S_{\tau}-\left(\text { ind } \mathfrak{L}_{\gamma}-\text { ind } \mathfrak{L}_{\beta}\right)-\left(\text { ind } \mathfrak{N}_{\gamma}-\text { ind } \mathfrak{N}_{\beta}\right),
$$

где $\pm S_{\tau}$ равен числу нулей концевого символа $\mathscr{X}_{j}(\zeta), \tau_{j}=\tau \in \mathscr{J}$ (с учетом кратности) между прямыми $\operatorname{Re} \zeta=\beta_{\tau}-r+1, \operatorname{Re} \zeta=\gamma_{\tau}-r+1$. Знак “-" соответствует случаю $\beta_{\tau} \leqslant \gamma_{\tau}$, а знак “+" - случаю $\beta_{\tau} \geqslant \gamma_{\tau}$.

Имеет место

СлЕДСТВИЕ 9.1. Пусть все корни характеристического полинома $P(\lambda)=$ $\operatorname{det} \mathscr{L}_{0}(x, \xi), \quad \xi=(-\lambda, 1)$, где $\mathscr{L}_{0}(x, \xi)-$ главная часть $\mathscr{L}(x, \xi)(c \mathcal{M} . \S 6)$, расположены или в верхней полуплоскости $\operatorname{Im} \lambda>0$, или в нижней. Тогда в первом случае в формуле $(9.23) \aleph=\bar{G}$, а концевой символ определяется равенством (5.6" $\left.{ }^{\prime \prime}\right)$, ге $\mathbf{a}=\mathbf{1}, \mathbf{b}-$ матрица (7.12), соответствующая (9.15). A во втором случае $=G$, а концевой символ определяется равенством (5.6' $\left.{ }^{\prime}\right)$, әде $\mathbf{a}=\mathbf{1}$, а $\mathbf{b}$ есть матрица (7.12), которая соответствует (9.15), (9.16), где коэффициенты $\mathbf{a}_{j}, \mathbf{b}_{j}$ заменены на комплексно сопряженные. (С учетом последнего определяется и матрица $G$.)

Следствие доказывается аналогично следствию 8.3.

b) Пусть теперь $\sigma_{0} \geqslant 0$. Тогда $k=t_{0}+\sigma_{0} \geqslant t_{0}$, поэтому здесь можно следовать п. $3 \S 8$. В соответствии с последним (после приведения задачи (9.12), (9.13) к виду $(9.15),(9.16))$ повысим порядок системы (9.15) до $k+1$. С этой целью нужно умножить слева оператор $\mathscr{A}_{1}$ задачи $(9.15),(9.16)$ последовательно на операторы $K_{V}, K_{V_{1}}, \ldots, K_{V_{k-t_{0}}}$. В результате мы получим оператор $\mathscr{A}_{2}$, являющийся оператором следуюшей задачи:

$$
\begin{aligned}
& \left(\frac{\partial}{\partial x_{1}}+i \frac{\partial}{\partial x_{2}}\right)^{\Delta} A v=f \in X_{(\beta-k-1)}^{n-\Delta}(Q), \quad v \in X_{(\beta)}^{n+t_{0}}(Q), \\
& \operatorname{Re}(B v)=\varphi \in Y_{(\beta)}^{n}(\partial Q \backslash \mathscr{J}), \\
& \operatorname{Re}(A v)=\varphi_{0} \in Y_{\left(\beta-t_{0}\right)}^{n}(\partial Q \backslash \mathscr{J}), \\
& \ldots \ldots \ldots \ldots \ldots \ldots \ldots \ldots \ldots \ldots \ldots \ldots \ldots \ldots \ldots \ldots \ldots \ldots \ldots \ldots \ldots \ldots \ldots \ldots \ldots \\
& \operatorname{Re}\left(\left(\frac{\partial}{\partial x_{1}}+i \frac{\partial}{\partial x_{2}}\right)^{k-t_{0}} A v\right)=\varphi_{k-r} \in Y_{(\beta-k)}^{n}(\partial Q \backslash \mathscr{J}),
\end{aligned}
$$


где $\Delta=k+1-t_{0}=\sigma_{0}+1, A v$ - левая часть (9.15). Причем из нётеровости $\mathscr{A}_{2}$ следует нётеровость опеатора $A_{1}$ при условии

$$
\beta_{\tau} \neq \frac{\pi}{\vartheta_{\tau}} n+j, \quad j=t_{0}, \ldots, t_{0}+\sigma_{0}+1, \quad \text { где } n \text { любое целое. }
$$

И в случае нётеровости индексы $\mathscr{A}_{2}$ и $\mathscr{A}_{1}$ связаны равенством (8.16): ind $\mathscr{A}_{1}=$ ind $\mathscr{A}_{2}-\varkappa_{2}$, где

$$
\varkappa_{2}=-\ell \sum_{j=r}^{k} \sum_{\tau \in \mathscr{J}}\left\{\left[\left(\beta_{\tau}-j\right) \frac{\vartheta_{\tau}}{\pi}\right]+1\right\}-\ell\left(\sigma_{0}+1\right)(m-1)
$$

Система (9.24) - однородная эллиптическая система порядка $k+1$. Краевые условия имеют порядок $k$, и их общее число равно $(k+1) \ell$. Выравним порядки всех краевых условий до $k$ оператором дифференцирований по дуге. Этот оператор нётеровый с индексом, равным

$$
\varkappa_{3}=\ell t_{0}\left(\sigma_{0}+1\right)|\mathscr{J}|+\frac{\ell\left(\sigma_{0}+1\right)}{2} \sigma_{0}|\mathscr{J}| .
$$

(Напомним, что $\sigma_{0}=\max \sigma_{i j}, t_{0}=\max t_{j}$.) Полученную таким образом задачу представим в виде (9.15), (9.16):

$$
\begin{gathered}
\sum_{j=1}^{r+1} \mathbf{a}_{j}^{0}\left(\frac{\partial}{\partial x_{2}}\right)^{r-j+1}\left(\frac{\partial}{\partial x_{1}}\right)^{j-1} v=f \in X_{\left(\beta-t_{0}\right)}^{n}(Q), \quad x \in Q, \\
\operatorname{Re} B v \equiv \operatorname{Re}\left(\sum_{j=1}^{r} \mathbf{b}_{j}^{0}\left(\frac{\partial}{\partial x_{2}}\right)^{r-j}\left(\frac{\partial}{\partial x_{1}}\right)^{j-1} v\right)=\varphi \quad \text { на } \quad \partial Q \backslash \mathscr{J},
\end{gathered}
$$

где $r=k+1=t_{0}+\sigma_{0}+1, \mathbf{a}_{1}^{0}, \ldots, \mathbf{a}_{r+1}^{0}-$ квадратные матрицы порядка $\ell$, $\mathbf{b}_{1}^{0}, \ldots, \mathbf{b}_{t_{0}}^{0}-(r \times \ell)$-матрицы. Определим матрицу $\mathbf{b}$ формулой (7.12). Разложим последнюю по формуле (2.1): $\mathbf{b}=T \operatorname{diag}\left(\Lambda_{1}, \Lambda_{2}\right) T^{-1}, T=\left(T_{1}, T_{2}\right)$. Пусть

$$
\begin{gathered}
\operatorname{det} \aleph_{0} \neq 0 \quad \text { всюду на } \quad \partial Q \backslash \mathscr{J}, \\
\operatorname{det} \aleph_{0}(\tau \pm 0) \neq 0, \quad \tau \in \mathscr{J},
\end{gathered}
$$

где $\aleph=\left(\bar{G}_{0} \bar{T}_{1}, G_{0} T_{2}\right), G_{0}=\left(\mathbf{b}_{1}^{0}, \ldots, \mathbf{b}_{r}^{0}\right)$. Задаче $(9.29),(9.30)$ поставим в соответствие задачу Римана-Гильберта для эллиптической системы $k=\ell(k+1)$ уравнений $\mathscr{D}_{1} u+\mathbf{b} \mathscr{D}_{2} u=g, \operatorname{Re}\left(G_{0} u\right)=\psi$, где $\mathbf{b}$ определена формулой (7.12).

Пусть $\mathscr{X}_{j}^{0}(\zeta), \tau_{j}=\tau \in \mathscr{J},-$ концевой символ этой задачи (он определен в п. $1 \S 5$; см. $\left.(5.6)-(5.8),\left(5.6^{\prime}\right),\left(5.6^{\prime \prime}\right)\right)$, и пусть

$$
\beta_{\tau}-t_{0}-\sigma_{0} \notin \mathbb{N} \cup\{0\}, \quad \tau \in \mathscr{J},
$$


где $\mathbb{N}$ - множество натуральных чисел. В силу теоремы 7.3 задача (9.29), (9.30) тогда и только тогда нётеровая, когда вьполнены (9.31) и условие

$$
\operatorname{det} \mathscr{X}_{j}^{0}(\zeta) \neq 0 \quad \text { на прямой } \quad \operatorname{Re} \zeta=\beta_{\tau}-t_{0}+1, \quad \tau=\tau_{j} \in \mathscr{J} .
$$

При выполнении этих условий индекс задачи дается равенством

$$
\text { ind } \mathscr{A}_{3}=\operatorname{Ind}_{\Gamma} \bar{\aleph}_{0}^{-1} \aleph_{0}-\sum_{j=1}^{|\mathscr{J}|} \operatorname{Ind}_{\beta_{j}-r+1} \mathscr{X}_{j}^{0}(\zeta) y_{j}^{-1}-\ell r(m-1) \text {. }
$$

Индексы задачи (9.29), (9.30) и (9.24), (9.25) связаны равенством

$$
\text { ind } \mathscr{A}_{3}=\text { ind } \mathscr{A}_{2}+\varkappa_{3} \text {. }
$$

Из всего этого следует, что справедлива

ТеОрема 9.2. Пусть выполнены условия (9.8), (9.19), (9.20), и пусть $\sigma_{0} \leqslant-1$, где $\sigma_{0}=\max \left\{\sigma_{i j}\right\}$ (см. (9.3)). Для нётеровости задачи (9.1), (9.2) необходимо и достаточно выполнения условий (9.17), (9.18). При выполнении этих условий индекс задачи дается формулой

$$
\text { ind } \mathscr{A}=\text { ind } \mathscr{A}_{3}-\left(\varkappa+\varkappa_{2}+\varkappa_{3}+\text { ind } \mathfrak{L}+\text { ind } \mathfrak{N}\right)
$$

где слагаемые справа определены формулами (9.9), (9.14), (9.22).

Теорема дополняется второй частью и следствием аналогично теореме 9.1.

2. Формула для индекса правильно эллиптических задач. Рассмотрим теперь задачу (6.3) для правильно эллиптической системы, где правые части и искомая принадлежат конечномерным расширениям пространств $(6.4),(6.5)$, как и в (9.1), (9.2):

$$
\begin{aligned}
& \mathscr{L}(x, \mathscr{D}) v=f \in \mathfrak{B}(Q), \quad v \in \mathfrak{A}(Q), \\
& \mathscr{B}(x, \mathscr{D}) v=\varphi \in \mathfrak{C}(\partial Q \backslash \mathscr{J}) .
\end{aligned}
$$

Предполагаем, что для (9.37) выполнены условия 1)-5) §6 (необходимые и достаточные для нётеровости (6.3), значит, и (9.37)). Задача (9.37) $\mathbb{C}$-линейная. Аналогично $\S 5$, перейдем от нее к $\mathbb{R}$-линейной задаче $(9.1),(9.2)$ с краевым условием $\operatorname{Re} \mathscr{B}^{0}(x, \mathscr{D}) v=\operatorname{Re} \operatorname{diag}(\mathscr{B}(x, \mathscr{D}),-i \mathscr{B}(x, \mathscr{D})) v=\psi, \psi=\left(\varphi_{1}, \varphi_{2}\right), \varphi_{1}+i \varphi_{2}=\psi \cdot \mathrm{B}$ качестве набора $\left\{\sigma_{i j}\right\}$, определенного формулой (9.3) (обозначим его здесь $\left\{\tilde{\sigma}_{i j}\right\}$ ), возьмем $\tilde{\sigma}_{i j}=\sigma_{i j}, \quad \tilde{\sigma}_{i+N^{\prime}, j}=\sigma_{i j}$ при $i=1, \ldots, N^{\prime}, N^{\prime}=N / 2$, и любом $j$, $j=1, \ldots,|\mathscr{J}|$, где $\left\{\sigma_{i j}\right\}$ - набор целых чисел, определенных в 5) $\S 6$. Полученную таким образом $\mathbb{R}$-линейную задачу представим в виде (9.15), (9.16).

Пусть $\aleph_{1}, \aleph_{2}, \mathscr{X}_{1 j}(\zeta), \mathscr{X}_{2 j}(\zeta)$ имеют тот же смысл, что и в теореме 7.1, и строятся по задаче $(9.15),(9.16)$. С учетом этого, аналогично теореме 5.2 , легко получим, что справедлива 
ТЕОРема 9.3. Пусть выполнены условия (9.8), (9.19), и пусть $\sigma_{0}=$ $\max \left\{\sigma_{i j}\right\} \leqslant-1$. Для нётеровости задачи (9.37) необходимо и достаточно выполнения условий (7.13), (7.14) и условия $\operatorname{det}\left(\mathscr{X}_{1 j}(\zeta), \mathscr{X}_{2 j}(\zeta)\right) \neq 0, \operatorname{Re} \zeta=$ $\beta_{\tau}-t_{0}+1, \tau=\tau_{j} \in \mathscr{J}$. При выполнении этих условий индекс задачи равен

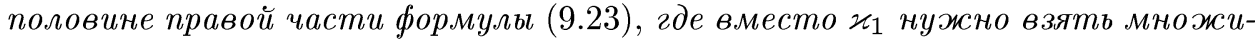
тель при $2^{-1}$ справа в $(7.16)\left(r=t_{0}\right)$.

В случае действительных коэффициентов во всех отмеченных формулах нужно взять $\mathscr{X}_{2 j}(\zeta)=\mathscr{X}_{1 j}(\zeta), \aleph_{2}=\aleph_{1}$.

Теорема дополняется и второй частью, аналогичной теореме 7.1.

Точно так же можно получить и аналог теоремы 9.2 , формулировку которой мы опускаем.

\section{Список литературы}

1. Радон И. О краевых задачах для логарифмического потенциала // УМН. 1946. Т. 1. № 3-4. C. 96-124.

2. Данилюк И. И. Нерегулярные граничные задачи на плоскости. М.: Наука, 1975.

3. Магнарадзе Л. Г. Основные задачи плоской теории упругости для контуров с угловыми точками // Тр. Тбилисск. матем. ин-та. 1938. Т. 4. С. 43-76.

4. Лопатинский Я. Б. Теория общих граничных задач. Киев: Наук. думка, 1984.

5. Солдатов А. П. Общая краевая задача $(k-1)$-го порядка для эллиптических уравнений // ДАН СССР. 1990. Т. 311. №1. С. 39-43.

6. Солдатов А. П. Общая краевая задача для эллиптических систем // ДАН СССР. 1990. T. 311. № 3. C. 539-543.

7. Солдатов А. П. Метод теории функций в эллиптических задачах на плоскости. 2. Кусочно гладкий случай // Изв. РАН. Сер. матем. 1992. Т. 56. № 3. С. 566-604.

8. Кондратьев В. А. Краевые задачи для эллиптических уравнений в областях с коническими и угловыми точками // Тр. Моск. матем. об-ва. 1967. Т. 16. С. 202-292.

9. Кондратьев B. А., Олейник O.A. Краевые задачи для уравнений с частными производными в негладких областях // УМН. 1983. Т. 38. №2. С. 3-76.

10. Назаров С. А., Пламеневский Б. А. Эллиптические задачи в областях с кусочно гладкой границей. М.: Наука, 1991.

11. Сиражудинов М. М. О задаче Римана-Гильберта для эллиптических систем первого порядка в многосвязной области // Матем. сб. 1993. Т. 184. № 11. С. 39-62.

12. Сиражудинов M. М. Краевые задачи для общих эллиптических систем на плоскости // Изв. РАН. Сер. матем. 1997. Т. 61. № 5. С. 137-176.

13. Солдатов А. П. Одномерные сингулярные операторы и краевые задачи теории функций. М.: Высшая школа, 1991.

14. Векуа И. Н. Обобщенные аналитические функции. М.: Наука, 1988.

15. Аәранович М. С., Вишик М. И. Эллиптические задачи с параметром и параболические задачи общего вида // УМН. 1964. Т. 49. №3. С. 53-160.

16. Крейн С. Г. Линейные уравнения в банаховом пространстве. М.: Наука, 1971.

17. Солонников B.A. Об общих краевых задачах для систем, эллиптических в смысле А. Дуглиса и Л. Ниренберга. I // Изв. АН СССР. 1964. Т. 28. №3. С. 665-706; II // Тр. Матем. ин-та им. В. А. Стеклова. 1966. Т. СІІ. С. 233-297.

18. Прёсдор $\$$ 3. Некоторые классы сингулярных уравнений. М.: Мир, 1979.

г. Махачкала, Дагестанский государственный университет, математический факультет
Поступило в редакцию 20.II.1998 